\title{
Casimir Effect on a Finite Lattice
}

\author{
A. Actor \\ Department of Physics, The Pennsylvania State University \\ Fogelsville, PA 18051, USA \\ E-mail: aaa2@psu.edu \\ I. Bender \\ Institut für Hochenergiephysik, Universität Heidelberg \\ Philosophenweg 16, D-69120 Heidelberg, Germany \\ E-mail: bender@thphys.uni-heidelberg.de \\ J. Reingruber \\ Institut für Theoretische Physik, Technische Universität München \\ James-Franck-Strasse, D-85748 Garching, Germany \\ E-mail: reingrub@physik.tu-muenchen.de
}

August 11, 2018

\begin{abstract}
Lattice quantum field theory is a well established branch of modern quantum field theory (QFT). However, it has only peripherally been used for the investigation of Casimir systems - i.e. for systems in which quantum fields are distorted by their interaction with classical background objects. This article presents a Hamiltonian lattice formulation of static Casimir systems at a level of generality appropriate for an introductory investigation. Background structure - represented by a lattice potential $V(x)$ - is introduced along one spatial direction with translation invariance in all other spatial directions. It is simple to extend this formulation to include arbitrary background structure in more than one spatial direction. Following some general analysis two specific finite 1D lattice QFT systems are analyzed in detail. The first has three Dirichlet boundaries at the lattice sites $x=0, l$ and $L(L>l>0)$ with vanishing lattice potential $V(x)$ everywhere in between. The vacuum energy and vacuum stress tensor $T^{\mu \nu}$ for this system are calculated in $0<x<L$. Very careful attention must be and is given to renormalization in the (continuum) limit of vanishing lattice constant. Globally and locally this lattice system is seen to closely mimic the corresponding $1 D$ continuum system - as one would hope. Then we introduce a lattice potential $V(x)=c /\left(x-x_{0}\right)^{2}$
\end{abstract}


centered at $x=x_{0}<0$ to the left of the boundary at $x=0$ and extending through this boundary and the middle Dirichlet boundary at $x=l$ out to the right-hand boundary $x=L>l$ and beyond. The vacuum energy and $T^{\mu \nu}$ are calculated for this far more complicated system in the region $0<x<L$, again with very good results. The internal consistency of the lattice version of this system is carefully examined. Our conclusion is that finite-lattice formulation provides a powerful and effective tool, capable of solving completely many Casimir systems which could not possibly be handled using continuum methods. This is precisely our reason for introducing it. Future investigations (in one and more dimensions and in dynamical as well as static contexts) will display more fully the power of this method. 


\section{Contents}

\begin{tabular}{lll}
\hline & Introduction & 1
\end{tabular}

2 Scalar field on a finite lattice with background 4

2.1 Continuum system . . . . . . . . . . . . . . . . 6

2.2 Lattice system . . . . . . . . . . . . . . . . . . . . . . . . . 9

2.2 .1 Quantization . . . . . . . . . . . . . . . 9

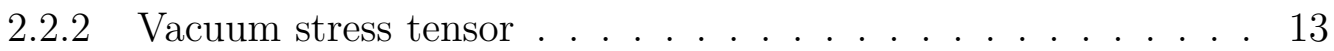

2.2 .3 The case $V\left(n_{1}\right)=0$. . . . . . . . . . . . . . . . . . . . . . . 16

3 One dimensional lattice without background potential 18

3.1 Renormalized vacuum energy . . . . . . . . . . . . . . 18

3.1 .1 Zero mass . . . . . . . . . . . . . . . . . . . . 18

3.1 .2 Nonzero mass . . . . . . . . . . . . . . . . . . . . . 20

3.2 Renormalized vacuum stress tensor . . . . . . . . . . . . . . . . . 24

3.2.1 $\left\langle T^{\Pi}\right\rangle \ldots \ldots \ldots \ldots \ldots \ldots \ldots$

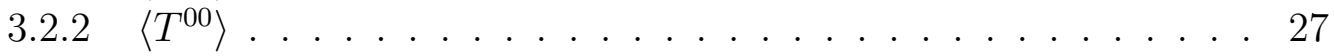

4 One dimensional lattice with Bessel potential background 30

4.1 Renormalized vacuum stress tensor for semihard $V(x)$. . . . . . . . . 31

$4.1 .1\left\langle T^{11}\right\rangle \ldots \ldots \ldots \ldots \ldots$

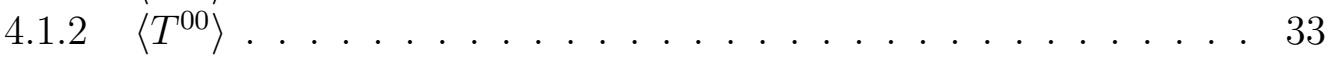

4.1 .3 Renormalized vacuum energy . . . . . . . . . . . . . . . . 33

4.2 Numerical evaluation using the Bessel potential . . . . . . . . . . . . 34

4.2 .1 Renormalized $\left\langle T^{00}\right\rangle \ldots \ldots \ldots \ldots$. . . . . . . . . . 34

4.2 .2 Renormalized $\left\langle T^{\Pi 1}\right\rangle$. . . . . . . . . . . . . . . . . . . 36

4.2 .3 Renormalized vacuum energy . . . . . . . . . . . . . . . . . . 39

4.2 .4 Casimir forces . . . . . . . . . . . . . . . . . . . . . . . . 40

$\begin{array}{lll}5 \text { Conclusion } & 45\end{array}$

\begin{tabular}{|lr}
\hline A Operators on the lattice & 47
\end{tabular}

A.1 Laplace operator . . . . . . . . . . . . . . . . . . . . . . . . . . 47

A.2 $\quad$ The operators $(\nabla \phi)^{2}$ and $\nabla(\phi \nabla \phi)$. . . . . . . . . . . . . . . . . . . . . . . 48

$\begin{array}{ll}\text { B A lattice artefact } & 51\end{array}$

C Perturbation theory on the one-dimensional lattice 56 


\section{Introduction}

Let us here define Casimir QFT (quantum field theory) to be the study of quantum fields in flat space, fields which coexist with and interact with classical background objects occupying the same space (in the simplest case Dirichlet or Neumann boundaries). This interaction distorts the quantum fields and all local observables connected with them away from the spatial uniformity (or translation invariance) that would characterize these fields in empty flat space. This nonuniformity in flat space caused by background objects is generally very pronounced, becoming even infinite as Dirichlet or Neumann boundary surfaces are approached. The background objects themselves experience measurable back forces - a subtle example of Newton's third law. These back forces are called Casimir forces and their presence is often identified with the Casimir effect. However the deeper essence of the Casimir effect is the distortion of the quantum fields caused by the background objects. Viewed this way the Casimir effect is obviously an extremely fundamental and general phenomenon in quantum field theory. Any nonuniform classical background whatsoever will produce a Casimir effect.

To the authors' knowledge all published work in Casimir QFT employs continuum QFT. In certain other branches of QFT, of course, lattice formulations have become commonplace. Quite well-developed methods exist for doing QFT on a spatial or spacetime lattice (see e.g. the book by Rothe [1]). Generally in lattice-QFT applications spatial uniformity is assumed. Either one chooses the spatial lattice to be infinite, or one imposes periodicity on a finite spatial lattice. There are no background objects on the lattice. Rather, one is using the lattice to learn about nonperturbative properties of interacting fields which are difficult if not impossible to investigate in continuum theory.

Some years ago two of the authors undertook the reformulation of Casimir QFT in lattice language. From the outset we have had two main objectives.

First, to gain a sense of how well lattice QFT works when applied to Casimir problems. By investigating a number of systems whose continuum versions can be solved explicitly we found that lattice QFT is able to mimic and reproduce in a quite detailed way both global and local features of continuum Casimir systems. While not unexpected this did need to be established quantitatively before we could proceed to our second and more important objective.

That objective is, to use lattice QFT to solve Casimir problems which cannot be dealt with (adequately, or even at all) using continuum QFT. It is unfortunate that practically all Casimir systems of physical interest belong in this latter difficult-toaccess category.

On a lattice one can work both analytically and numerically. Analytic methods tend to rather closely parallel continuum theory and, while interesting, do not necessarily offer greatly increased computational power, especially on an infinite lattice. Numerical methods used on a finite lattice can be, however, very powerful. One can introduce static background objects on the finite spatial lattice and make the 
quantum field $\phi(x)$ interact with these objects, the interaction being described by a potential in a functional Schrödinger equation, whose stationary solutions are expressible in terms of the eigenfunctions and the spectrum of an ordinary Schrödinger equation associated with the functional one. These spatial modes and the spectrum can be found numerically. Given these basic ingredients one can then compute everything numerically - the vacuum energy and the stress-energy-momentum tensor, Casimir forces and so on. This can surely be done for a vast array of systems which could not be solved in continuum theory.

Of course renormalization must be performed to make lattice observables physically meaningful. Everything one calculates on a finite lattice is finite. However, lattice quantum variables like vacuum energy and the vacuum expectation value of the stress-energy-momentum tensor $T^{\mu \nu}$ contain the seeds of ultraviolet (UV) divergences, i.e. terms which grow without limit in the continuum limit $a \rightarrow 0$ ( $a$ is the lattice constant or spacing between lattice points). For finite and decreasing $a$ these essentially informationless terms eventually overwhelm the physical signal: their systematic removal is utterly essential. Lattice quantities which vanish as $a \rightarrow 0$ take care of themselves, of course. What remains is the "physical signal" a contribution independent of $a$ which therefore neither blows up nor vanishes as $a \rightarrow 0$. All of this is the process of renormalization.

For a number of years we have pursued this numerical strategy, for both standard and unconventional Casimir systems. To date none of this lattice QFT work has been reported (excepting the brief descriptions [2, 3]). We begin here the systematic development of lattice QFT with background objects and its application to nontrivial Casimir systems. Before describing the contents of the present article we pause to mention some nonstandard aspects of Casimir QFT.

Traditional Casimir systems have "hard" boundary surfaces - i.e. boundary surfaces $\partial \mathcal{M}$ with precise spatial location on which the quantum field $\phi(x)$ has to satisfy some boundary condition. A perhaps more physically realistic boundary can be fashioned by attaching to a Dirichlet boundary $\partial \mathcal{M}$ (on which $\phi(x)$ has to vanish) a static potential $V(x)$ which grows to infinity as $x$ approaches $\partial \mathcal{M}$ and falls off to zero more or less rapidly away from $\partial \mathcal{M}$. The interaction of this classical potential with the quantum field $\phi(x)$ is for simplicity assumed to be described by an interaction term quadratic in $\phi(x):(1 / 2) \phi^{2}(x) V(x)$. We refer to such a modification of a hard Dirichlet surface as semihardening [4, 5]. The potential function $V(x)$ represents (one can say) surface texture attached to $\partial \mathcal{M}$. The wave equation $\left[\partial_{0}^{2}-\Delta+m^{2}\right] \phi=0$ in usual hard-boundary scalar-field Casimir QFT with appropriate boundary conditions on $\phi$ is replaced by

$$
\left[\partial_{0}^{2}-\Delta+m^{2}+V(x)\right] \phi(x)=0
$$

Here $V(x)$ should be viewed as a (rather huge) generalization of ordinary boundary conditions. This potential can represent many kinds of background structure which interact with $\phi$ and are (for $V(x)<\infty$ ) partially transparent to $\phi$. The semihardening potentials above are just one kind of background spatial structure 
among many which can be introduced into QFT (see e.g. refs. [6, 8, 14, 15]). For us, Casimir QFT comprises all possible background structures interacting with quantum fields - a truly vast subject. Many workers, of course, use the term "Casimir effect" mainly in connection with boundary surfaces (see e.g. the reviews [8, 16]). Diffuse background structures in QFT are then often called "background fields".

As mentioned earlier the fundamental phenomenon in Casimir QFT is the distortion of otherwise spatially uniform fields and observable quantities away from spatial uniformity by the background $V(x)$. To really understand a Casimir system one must work locally. There is no better physical quantity to compute than the vacuum expectation value of the stress-energy-momentum tensor (hereafter stress tensor) of a Casimir system which we will denote by $\left\langle T^{\mu \nu}(x)\right\rangle$ in the following. This quantity reveals the spatial nonuniformity of the system, its nonuniform vacuum energy density and its Casimir forces. $\left\langle T^{\mu \nu}(x)\right\rangle$ can be computed from the spatial modes and spectrum of $\phi(x)$, both in continuum theory and on a lattice. When working numerically on a finite lattice (using a lattice potential $V(x)$ ) one can obtain $\left\langle T^{\mu \nu}(x)\right\rangle$ numerically for practically any kind of background structure.

In sec. 2 we begin our analysis of Casimir systems of the following type: A space of dimension $d$ is chosen with free (or periodic) "boundary conditions" in each of the directions $x_{2}, \cdots, x_{d}$. Along the $x_{1}$ axis we place parallel Dirichlet boundaries at $x_{1}=0$ and $x_{1}=l$. In sec. 2 we do not concern ourselves with the regions $x_{1}<0$ and $x_{1}>l$ external to the interval $0 \leq x_{1} \leq l$ of interest. These external regions can be "attached" later on and given suitable global structure (e.g. periodicity in $x_{1}$ over some larger interval) to complete the specification of the physical system. We postpone doing this without loss of generality. In the interval $0 \leq x_{1} \leq l$ we introduce an arbitrary background potential $V\left(x_{1}\right)$. The formulation of lattice scalar QFT on this spatial manifold is reviewed in detail in sec. 2, where detailed lattice formulae for $\left\langle T^{\mu \nu}\right\rangle$ are given and the ground is prepared for our very specific lattice analyses to follow in secs. 3,4 .

In sec. 3 we go to one dimension $d=1$, set the background potential $V(x)=0$ and study scalar lattice QFT on a $1 D$ lattice $0 \leq x \leq l$ with Dirichlet walls at $x=0, l$ and no structure in between. Choosing lattice constant $a$ means $l=N a, x=n a$ where $n=0,1, \cdots, N$ labels the lattice sites. The continuum limit $a \rightarrow 0$ (with $l$ fixed of course) means $N=l / a \rightarrow \infty$. As described above we must identify and subtract those terms in the lattice vacuum energy and $\left\langle T^{\mu \nu}\right\rangle$ of this system which diverge for $a \rightarrow 0$. The remaining $a$-independent parts of these functions are then to be brought into comparison with continuum theory. In sec. 3 we present detailed analysis of this continuum limit on the $1 D$ lattice. To enable us to make definite statements about the Casimir force on the boundary $x=l$ we introduce an "external" region $l \leq x \leq L$ with $L \gg l$ which can be analyzed on the lattice exactly as we do the "internal" region $0 \leq x \leq l$. We then have a more complete physical system than just the internal region $0 \leq x \leq l$. To make it a re- 
ally complete physical system the region $x<0$ needs to be included and the global structure of the $x$ axis must be specified. However there is no need to do all this here. Our goal is to show how well hard-boundary renormalized lattice QFT mimics continuum QFT. That goal is nicely achieved using the two adjacent $1 D$ lattices $0 \leq x \leq l, l \leq x \leq L$.

Sec. 4 tackles a more difficult problem. Keeping spatial dimension $d=1$ we introduce the Bessel semihardening potential $V(x)=c /\left(x-x_{0}\right)^{2}$ centered at $x=x_{0}<0$, allowing this potential to extend arbitrarily far into $x>0$. Immersed in $V(x)$ we position Dirichlet point boundaries at $x=0, l, L$ as before. One can find the lattice vacuum energy $E^{v a c}$ and $\left\langle T^{\mu \nu}\right\rangle$ numerically for this system with arbitrary background potential $V(x)$. In sec. 4.1 we show how to renormalize $E^{v a c}$ and $\left\langle T^{\mu \nu}\right\rangle$ for arbitrary $V(x)$. Then we adopt the Bessel potential above and begin our principal numerical analysis. In a sense the numerical work in sec. 4.2 is the central part of this article. Using $E_{\text {ren }}^{v a c}$ we calculate globally the Casimir force on the middle boundary $x=l$ due to the other Dirichlet boundaries and the potential $V(x)$. Using $\left\langle T^{\mu \nu}\right\rangle_{\text {ren }}$ we calculate locally the same Casimir force. Very precise agreement is found. Some other things are computed as well, but let us limit our description of sec. 4 to the comment just made. This agreement between global and local calculations of a physical Casimir force in a quite nontrivial background potential gives us great confidence in the methods we are using. We have no doubt that equally good results can be gotten for practically any background $V(x)$. Indeed we have had comparable success with other potentials.

Concluding remarks are made in sec. 5. There we indicate the directions in lattice QFT theory we presently are pursuing. Three appendices dealing with technical matters enable us to present a substantially condensed narrative.

\section{Scalar field on a finite lattice with background}

To construct our Casimir system we begin with flat d-dimensional space and give it background structure along just one direction, say $x_{1}$. The other directions $\vec{x}_{\perp}=\left(x_{2}, \cdots, x_{d}\right)$ are free but - in preparation for the finite lattice - we make each of these coordinates periodic in $-L \leq x_{j} \leq L$ with period $2 L$. Periodicity is, of course, closely akin to "free boundary conditions", neither of these being a true boundary condition at all. Thus $\vec{x}_{\perp}$ specifies position on a spatial $(d-1)$-dimensional torus $T^{d-1}$ representing the free directions and $x=\left(x_{1}, \vec{x}_{\perp}\right)$ is position in space. A real scalar field $\phi(x)$ with mass $m$ is now defined on this space. $\phi(x)$ interacts with and is made nonuniform by and exerts a back force on the background structure along the $x_{1}$ direction. This interaction is what we wish to study in detail.

For completeness let us mention something we do not wish to study more than nec- 
essary: the so-called "topological Casimir effect". When a quantum field $\phi(x)$ is defined on a topologically nontrivial space, $\phi(x)$ is forced to be (nontrivially) different than it would be in free infinite space. This is itself a kind of Casimir effect. The vacuum energy density of $\phi(x)$ is shifted, vacuum stresses or Casimir forces are exerted by the modified field directly on the spatial manifold causing the modification etc (see e.g. the reviews [8, 16]). When, as here, one defines one's field on a (d-1)-dimensional torus $T^{d-1}$ (the periodic directions $x_{2}, \cdots, x_{d}$ ) there arises a topological Casimir effect parametrized by the finite circumferences of $T^{d-1}$. However, no "objects" have been introduced anywhere; periodic boundary conditions do not involve real boundaries. The $T^{d-1}$ topological Casimir effect smoothly disappears as the torus circumferences become infinite.

Topological Casimir effects caused by global spatial structure coexist with the field distortion caused by locally nonuniform spatial backgrounds, or objects in space. One should, however, make the effort to keep these two aspects of QFT distinct in one's thinking. This seems particularly important when one replaces continuum QFT by lattice QFT. For numerical work the lattice has to be finite, inevitably introducing the topological effect under discussion, which lattice workers and others usually call "finite-size effects". Unless one wishes specifically to study them, finitesize effects tend for obvious reasons to be regarded as a nuisance.

Many readers may be familiar with finite-size scaling theory (see e.g. refs [17, 18, 19]) in the general area of statistical systems. This and other related work one finite-size effects in QFT has rather limited overlap with and relevance for the kind of investigation we are concerned with here. Our focus is on the distorting effect background objects have on quantum fields. Finite-size scaling theory usually deals with bulk effects and large scale collective phenomena near critical points, and the effect of small system size on such phenomena. Phase transitions are involved, and temperature plays an essential role. In Casimir QFT temperature is something of a sideshow (although not completely without interest), and phase transitions normally play no role whatsoever. True, the effects of surfaces and corners in statistical models are sometimes taken into account (see e.g. [17]), and this overlaps with our work more substantially. However, an attempt to pursue this would take us far outside the scope of the present work, and necessitate substantially increased length in an already long paper.

Resuming now our mathematical discussion, we position along $x_{1}$ two planar Dirichlet boundaries at $x_{1}=0$ and $x_{1}=l$. Between these boundaries we subject the quantum field $\phi(x)$ to a background potential $V\left(x_{1}\right) \geq 0$. Lacking external regions $x_{1}<0$ and $x_{1}>l$ this Casimir system is physically incomplete. However it is easy to attach these external regions. As long as one does not try to discuss Casimir forces on the boundaries $x_{1}=0, l$ there is no need to be concerned about anything outside $0<x<l$. Our purpose in this section will be the detailed formulation of the lattice quantum theory in $0<x<l$ in the presence of arbitrary background potential $V\left(x_{1}\right)$. We begin with a brief summary of the continuum QFT we wish to put on a spatial lattice. 


\subsection{Continuum system}

The classical Lagrangian of the real scalar field interacting with a background, represented by a classical potential $V\left(x_{1}\right)$ becoming infinite at $x_{1}=0, l$, is

$$
\begin{aligned}
L=\int d^{d} x \mathcal{L} & =\int d^{d} x \frac{1}{2}\left(\left(\partial^{\mu} \phi\right)\left(\partial_{\mu} \phi\right)-\left(m^{2}+V\left(x_{1}\right)\right) \phi^{2}\right) \\
& =\int d^{d} x \frac{1}{2}\left(\dot{\phi}^{2}-\phi\left(m^{2}+V\left(x_{1}\right)-\triangle\right) \phi\right)
\end{aligned}
$$

Here in the partial integration used to reach the final equality the surface term vanishes because of the boundary conditions imposed on the classical field $\phi(x, t)$ :

$$
\begin{aligned}
\phi\left(x_{1}=0, \vec{x}_{\perp}, t\right) & \left.=\phi\left(x_{1}=l, \vec{x}_{\perp}, t\right)=0 \quad \text { (due to } V(0)=V(l)=\infty\right) \\
\phi\left(x_{1}, \vec{x}_{\perp}, t\right) & =\phi\left(x_{1}, \vec{x}_{\perp}+2 L \vec{e}_{i}, t\right), \quad i=2, \ldots, d \quad \text { (periodicity) }
\end{aligned}
$$

$\vec{e}_{i}$ being the unit vector in i-direction. Using the canonically conjugate field

$$
\pi(x, t) \equiv \frac{\delta L}{\delta \dot{\phi}(x, t)}=\dot{\phi}(x, t)
$$

we obtain from $L$ by Legendre transformation the classical Hamiltonian

$$
H=\int d^{d} x \pi(x, t) \dot{\phi}(x, t)-L=\int d^{d} x \frac{1}{2}\left(\pi^{2}+\phi\left(m^{2}+V\left(x_{1}\right)-\triangle\right) \phi\right) \quad .
$$

Canonical quantization means replacing the classical field $\phi(x, t)$ and its conjugate momentum $\pi(x, t)$ by hermitian operators $\Phi(x, t)$ and $\Pi(x, t)$ which obey the equaltime commutation relations

$$
\begin{aligned}
{\left[\Phi(x, t), \Phi\left(x^{\prime}, t\right)\right]=\left[\Pi(x, t), \Pi\left(x^{\prime}, t\right)\right] } & =0, \\
{\left[\Phi(x, t), \Pi\left(x^{\prime}, t\right)\right] } & =i \delta\left(x-x^{\prime}\right) .
\end{aligned}
$$

Following standard procedure one can realize these commutation relations by expanding the field operator in terms of creation and annihilation operators $\hat{a}_{k}^{\dagger}$ and $\hat{a}_{k}$ which satisfy $\left[\hat{a}_{k}, \hat{a}_{k^{\prime}}^{\dagger}\right]=\delta_{k, k^{\prime}}$ and $\left[\hat{a}_{k}, \hat{a}_{k^{\prime}}\right]=\left[\hat{a}_{k}^{\dagger}, \hat{a}_{k^{\prime}}^{\dagger}\right]=0$;

$$
\hat{\phi}(x, t)=\sum_{k} \frac{1}{\sqrt{2 \epsilon_{k}}}\left[\hat{a}_{k} e^{-i \epsilon_{k} t} u_{k}(x)+\hat{a}_{k}^{\dagger} e^{i \epsilon_{k} t} u_{k}^{*}(x)\right] .
$$

Here the $u_{k}(x)$ and $\epsilon_{k}$ are eigenfunctions and the associated eigenvalues of the operator $\left[-\Delta+m^{2}+V\left(x_{1}\right)\right]$, i.e.

$$
\left[m^{2}+V\left(x_{1}\right)-\triangle\right] u^{k}(x)=\varepsilon_{k}^{2} u^{k}(x) .
$$


Separating variables

$$
\begin{aligned}
u^{k}(x)=v^{k_{1}}\left(x_{1}\right) w^{k_{2}}\left(x_{2}\right) \cdots w^{k_{d}}\left(x_{d}\right) & =v^{k_{1}}\left(x_{1}\right) w^{\vec{k}_{\perp}}\left(\vec{x}_{\perp}\right) \\
k=\left(k_{1}, k_{2}, \ldots, k_{d}\right) & =\left(k_{1}, \vec{k}_{\perp}\right)
\end{aligned}
$$

leads to

$$
\begin{aligned}
\left(V\left(x_{1}\right)-\frac{\partial^{2}}{\left(\partial x_{1}\right)^{2}}\right) v^{k_{1}}\left(x_{1}\right) & =\rho_{k_{1}}^{2} v^{k_{1}}\left(x_{1}\right) \\
-\frac{\partial^{2}}{\left(\partial x_{i}\right)^{2}} w^{k_{i}}\left(x_{i}\right) & =\omega_{k_{i}}^{2} w^{k_{i}}\left(x_{i}\right), \quad i=2, \ldots, d \text { and } \\
\varepsilon_{k}^{2} & =m^{2}+\rho_{k_{1}}^{2}+\sum_{i=2}^{d} \omega_{k_{i}}^{2},
\end{aligned}
$$

where the boundary conditions on $v^{k_{1}}$ and $w^{k_{i}}$ are

$$
\begin{aligned}
v^{k_{1}}(0) & =v^{k_{1}}(l)=0, \\
w^{k_{i}}\left(x_{i}\right) & =w^{k_{i}}\left(x_{i}+2 L\right), \quad i=2, \ldots, d .
\end{aligned}
$$

Obviously for the free directions

$$
w^{k_{i}}\left(x_{i}\right)=\sqrt{\frac{1}{2 L}} e^{i \frac{\pi}{L} k_{i} x_{i}}
$$

with eigenvalues

$$
\omega_{k_{i}}^{2}=\frac{\pi^{2}}{L^{2}} k_{i}^{2}, \quad k_{i} \in \mathbf{Z}
$$

The functions $v^{k_{1}}\left(x_{1}\right)$ of course depend on the potential $V\left(x_{1}\right)$. These functions are chosen to be real.

Altogether we have

$$
\begin{aligned}
u^{k}(x) & =v^{k_{1}}\left(x_{1}\right) w^{\vec{k}_{\perp}}\left(\vec{x}_{\perp}\right)=v^{k_{1}}\left(x_{1}\right)\left(\frac{1}{2 L}\right)^{\frac{d-1}{2}} e^{i \frac{\pi}{L} \vec{k}_{\perp} \vec{x}_{\perp}}, \\
\varepsilon_{k}^{2} & =m^{2}+\rho_{k_{1}}^{2}+\sum_{i=2}^{d} \frac{\pi^{2}}{L^{2}} k_{i}^{2} .
\end{aligned}
$$

The set of modes $\left\{u^{k}(x)\right\}$ is complete and orthonormal

$$
\sum_{k} u^{k}(x) u^{k^{*}}\left(x^{\prime}\right)=\bar{\delta}\left(x-x^{\prime}\right) \quad \text { and } \quad \int d^{d} x u^{k}(x) u^{k^{*}}(x)=\delta_{k, k^{\prime}}
$$

where

$$
\sum_{k} \ldots=\sum_{k_{1}=1}^{\infty} \sum_{k_{2}=-\infty}^{\infty} \cdots \sum_{k_{d}=-\infty}^{\infty} \cdots \quad, \quad \delta_{k, k^{\prime}}=\delta_{k_{1}, k_{1}^{\prime}} \delta_{k_{2}, k_{2}^{\prime}} \cdots \delta_{k_{d}, k_{d}^{\prime}}
$$


Due to periodicity in $x_{2}, \cdots, x_{d}$ we need to introduce here the periodic $\delta$-function $\tilde{\delta}(x)$ along these directions, distinguished from the usual $\delta$-function for a noncompact direction by its tilde. The spatial $\delta$-function is then

$$
\bar{\delta}\left(x-x^{\prime}\right)=\delta\left(x_{1}-x_{1}^{\prime}\right) \tilde{\delta}\left(\vec{x}_{\perp}-\vec{x}_{\perp}^{\prime}\right) .
$$

Our attention now shifts to the vacuum stress (energy-momentum) tensor of the system. The classical canonical stress tensor for a real scalar field is

$$
T^{\mu \nu}=\frac{1}{2}\left(\left(\partial^{\mu} \phi\right)\left(\partial^{\nu} \phi\right)+\left(\partial^{\nu} \phi\right)\left(\partial^{\mu} \phi\right)\right)-g^{\mu \nu} \mathcal{L}
$$

with $\mathcal{L}$ given by the first equality in eq. (2.1). Again standard procedure (see e.g. [10]) leads to the following mode-sum formulae for the vacuum expectation value of the operator $T^{\mu \nu}$ :

$$
\begin{aligned}
\left\langle 0\left|T^{00}(x)\right| 0\right\rangle & =\sum_{k} \frac{\varepsilon_{k} v^{k_{1}}\left(x_{1}\right)^{2}}{2(2 L)^{d-1}} \\
& +\sum_{k} \frac{1}{4 \varepsilon_{k}(2 L)^{d-1}} \frac{\partial}{\partial x_{1}}\left(v^{k_{1}}\left(x_{1}\right) \frac{\partial v^{k_{1}}\left(x_{1}\right)}{\partial x_{1}}\right) \\
& =\sum_{k} \frac{\left(2 \varepsilon_{k}^{2}-\rho_{k_{1}}^{2}+V\left(x_{1}\right)\right) v^{k_{1}}\left(x_{1}\right)^{2}}{4 \varepsilon_{k}(2 L)^{d-1}} \\
& +\sum_{k} \frac{1}{4 \varepsilon_{k}(2 L)^{d-1}}\left(\frac{\partial v^{k_{1}}\left(x_{1}\right)}{\partial x_{1}}\right)^{2}, \\
\left\langle 0\left|T^{11}(x)\right| 0\right\rangle & =\sum_{k} \frac{\left(\rho_{k_{1}}^{2}-V\left(x_{1}\right)\right) v^{k_{1}}\left(x_{1}\right)^{2}}{2 \varepsilon_{k}(2 L)^{d-1}} \\
& +\sum_{k} \frac{1}{4 \varepsilon_{k}(2 L)^{d-1}} \frac{\partial}{\partial x_{1}}\left(v^{k_{1}}\left(x_{1}\right) \frac{\partial v^{k_{1}}\left(x_{1}\right)}{\partial x_{1}}\right) \\
& =\sum_{k} \frac{\left(\rho_{k_{1}}^{2}-V\left(x_{1}\right)\right) v^{k_{1}}\left(x_{1}\right)^{2}}{4 \varepsilon_{k}(2 L)^{d-1}}+\sum_{k} \frac{1}{4 \varepsilon_{k}(2 L)^{d-1}}\left(\frac{\partial v^{k_{1}}\left(x_{1}\right)}{\partial x_{1}}\right)^{2}
\end{aligned}
$$

and for $i=2, \ldots, d$

$$
\begin{aligned}
\left\langle 0\left|T^{i i}(x)\right| 0\right\rangle & =\sum_{k} \frac{\omega_{k_{i}}^{2} v^{k_{1}}\left(x_{1}\right)^{2}}{2 \varepsilon_{k}(2 L)^{d-1}} \\
& -\sum_{k} \frac{1}{4 \varepsilon_{k}(2 L)^{d-1}} \frac{\partial}{\partial x_{1}}\left(v^{k_{1}}\left(x_{1}\right) \frac{\partial v^{k_{1}}\left(x_{1}\right)}{\partial x_{1}}\right) \\
& =\sum_{k} \frac{\left(\rho_{k_{1}}^{2}-V\left(x_{1}\right)+2 \omega_{k_{i}}^{2}\right) v^{k_{1}}\left(x_{1}\right)^{2}}{4 \varepsilon_{k}(2 L)^{d-1}} \\
& -\sum_{k} \frac{1}{4 \varepsilon_{k}(2 L)^{d-1}}\left(\frac{\partial v^{k_{1}}\left(x_{1}\right)}{\partial x_{1}}\right)^{2} .
\end{aligned}
$$


The first equality in eq. (2.22) shows that

$$
\int d x\left\langle 0\left|T^{00}\right| 0\right\rangle=\frac{1}{2} \sum_{k} \epsilon_{k}
$$

as one expects.

\section{$2.2 \quad$ Lattice system}

The Casimir system will now be redefined on a finite lattice, which is given the same lattice constant $a$ in all spatial directions. Thus spatial position becomes $x=$ $\left(x_{1}, \cdots, x_{d}\right)=a\left(n_{1}, \cdots, n_{d}\right)$ where $n_{1}, \cdots, n_{d}$ all take integer values. The planar Dirichlet boundaries at $x_{1}=0, l$ have lattice positions $n_{1}=0$ and $n_{1}=l / a \equiv N_{1}$. The continuum potential $V\left(x_{1}\right)$ becomes a lattice potential $V\left(n_{1}\right) \geq 0$. The free directions $\vec{x}_{\perp}=a \vec{n}_{\perp}$ are still periodic with period $2 L$ :

$$
-L \leq x_{i} \leq L \quad \longrightarrow \quad-\frac{L}{a}<n_{i} \leq \frac{L}{a}=N_{\perp}, \quad i=2, \ldots, d,
$$

i.e. the lattice is periodic with period $2 N_{\perp}$ in the spatial directions $i=2, \cdots, d$. On the lattice the Dirac $\delta$-function $\delta\left(x_{1}-x_{1}^{\prime}\right)$ is replaced by $\delta_{n_{1}, n_{1}^{\prime}} / a$ with $\delta_{n_{1}, n_{1}^{\prime}}$ the usual Kronecker $\delta$. Delta functions $\delta_{n_{i} n_{i}^{\prime}}$ in the periodic directions $i>1$ are periodic of course.

\subsubsection{Quantization}

We now quantize the lattice Casimir system carefully and in detail, using the Schrödinger picture. On the lattice the classical field $\phi(x, t)$ becomes

$$
\phi(x, t)=\phi\left(x_{1}, \vec{x}_{\perp}, t\right) \longrightarrow \phi_{n}(t)=\phi_{n_{1}, \vec{n}_{\perp}}(t) .
$$

subject to the boundary conditions

$$
\begin{aligned}
\phi_{0, \vec{n}_{\perp}}(t) & =\phi_{N_{1}, \vec{n}_{\perp}}(t)=0, \\
\phi_{n_{1}, \vec{n}_{\perp}}(t) & =\phi_{n_{1}, \vec{n}_{\perp}+2 N_{\perp} \vec{e}_{i}}(t), \quad i=2, \ldots, d .
\end{aligned}
$$

As before we ignore the external regions $n_{1}<0$ and $n_{1}>N_{1}=l / a$ beyond the Dirichlet planes. Since $\phi \equiv 0$ on these planes the interior lattice $G$ on which $\phi(x)$ is a quantum field does not include these planes:

$$
G=\left\{n \in \mathbf{Z}^{d} \mid 1 \leq n_{1} \leq\left(N_{1}-1\right) ;\left(-N_{\perp}+1\right) \leq n_{i} \leq N_{\perp}, \quad i=2, \ldots d\right\} .
$$

From eq. (2.1) we obtain the classical Lagrangian

$$
\begin{aligned}
L & =\frac{1}{2}\left\{\sum_{n_{1}=0}^{N_{1}-1}+\sum_{n_{1}=1}^{N_{1}}\right\} \sum_{n_{2}=-N_{\perp}+1}^{N_{\perp}} \ldots \sum_{n_{d}=-N_{\perp}+1}^{N_{\perp}} \frac{a^{d}}{2}\left(\dot{\phi}_{n}^{2}-\phi_{n}\left(m^{2}+V\left(n_{1}\right)\right) \phi_{n}-(\nabla \phi)_{n}^{2}\right) \\
& =\sum_{n \in G} \frac{a^{d}}{2}\left(\dot{\phi}_{n}^{2}-\phi_{n}\left(m^{2}+V\left(n_{1}\right)\right) \phi_{n}+\phi_{n}(\triangle \phi)_{n}\right),
\end{aligned}
$$


where

$$
\sum_{n \in G} \ldots=\sum_{n_{1}=1}^{N_{1}-1} \sum_{n_{2}=-N_{\perp}+1}^{N_{\perp}} \cdots \sum_{n_{d}=-N_{\perp}+1}^{N_{\perp}} \cdots
$$

and $\triangle=\triangle_{n, n^{\prime}}$ is the lattice Laplace operator (see Appendix A). The specific $n_{1}$-summation prescription given in (2.30) avoids introducing an asymmetry in the $x_{1}$-direction by the asymmetry of the lattice derivative. The classical canonical momenta are

$$
\pi_{n}(t)=\frac{\partial L}{\partial \dot{\phi}_{n}(t)}=a^{d} \dot{\phi}_{n}(t), \quad \forall n \in G
$$

and the classical Hamiltonian is

$$
H=\sum_{n \in G} \pi_{n}(t) \dot{\phi}_{n}(t)-L=\sum_{n \in G} \frac{a^{d}}{2}\left(\frac{\pi_{n}^{2}}{a^{2 d}}+\phi_{n}\left(m^{2}+V\left(n_{1}\right)\right) \phi_{n}-\phi_{n}(\triangle \phi)_{n}\right)
$$

Canonical quantization in the Schrödinger picture assigns to the classical field $\phi_{n}$ and momentum $\pi_{n}$ time independent operators

$$
\begin{aligned}
& \phi_{n}(t) \longrightarrow \Phi_{n}, \\
& \frac{\pi_{n}(t)}{a^{d}} \longrightarrow \Pi_{n},
\end{aligned}
$$

which satisfy the commutation relations

$$
\begin{gathered}
{\left[\Phi_{n}, \Phi_{n^{\prime}}\right]=\left[\Pi_{n}, \Pi_{n^{\prime}}\right]=0,} \\
{\left[\Phi_{n}, \Pi_{n^{\prime}}\right]=\frac{i}{a^{d}} \delta_{n, n^{\prime}} .}
\end{gathered}
$$

Note that in the continuum limit $a \rightarrow 0$

$$
\begin{aligned}
\frac{1}{a^{d}} \delta_{n, n^{\prime}} & \rightarrow \delta\left(x-x^{\prime}\right) \\
\Pi_{n} & \rightarrow \Pi(x) .
\end{aligned}
$$

The lattice Hamilton operator is

$$
\mathcal{H}=\sum_{n \in G} \frac{a^{d}}{2}\left(\Pi_{n}^{2}+\Phi_{n}\left(m^{2}+V\left(n_{1}\right)\right) \Phi_{n}-\Phi_{n}(\triangle \Phi)_{n}\right) .
$$

We go now to the field representation in which the field operators $\Phi_{n}$ are simply multiplicative:

$$
\Phi_{n} \Psi\left[\left\{\phi_{m}\right\}, t\right]=\phi_{n} \Psi\left[\left\{\phi_{m}\right\}, t\right] .
$$

Here $\Psi\left[\left\{\phi_{m}\right\}, t\right]$ is the lattice analogue of the wave functional $\Psi[\phi, t]$ of continuum theory, i.e. a function of the c-number variables $\phi_{m}$. The canonical-momentum 
operators $\Pi_{n}$ become the differential operators $-\left(i / a^{d}\right) \partial / \partial \phi_{n}$. In this representation the Hamilton operator assumes the form

$$
\begin{aligned}
\mathcal{H} & =\sum_{n \in G}\left(-\frac{1}{2 a^{d}} \frac{\partial^{2}}{\left(\partial \phi_{n}\right)^{2}}+\frac{a^{d}}{2} \phi_{n}\left(m^{2}+V\left(n_{1}\right)\right) \phi_{n}-\phi_{n}(\triangle \phi)_{n}\right) \\
& =\sum_{n \in G}\left(-\frac{1}{2 a^{d}} \frac{\partial^{2}}{\left(\partial \phi_{n}\right)^{2}}+\frac{a^{d}}{2} \phi_{n} \sum_{n^{\prime} \in G} \mathcal{O}_{n, n^{\prime}} \phi_{n^{\prime}}\right)
\end{aligned}
$$

with (see eq. (A.9) )

$$
\begin{aligned}
\mathcal{O}_{n, n^{\prime}} & =\left(m^{2}+V\left(n_{1}\right)\right) \delta_{n, n^{\prime}}-\triangle_{n, n^{\prime}} \\
& =\left(m^{2}+V\left(n_{1}\right)\right) \delta_{n, n^{\prime}}-\sum_{j=1}^{d} \frac{1}{a^{2}}\left(\delta_{n+\vec{e}_{j}, n^{\prime}}-2 \delta_{n, n^{\prime}}+\delta_{n-\vec{e}_{j}, n^{\prime}}\right) .
\end{aligned}
$$

This matrix is symmetric in $\left(n, n^{\prime}\right)$ and positive definite. We need its eigenvectors and eigenvalues to diagonalize the Hamilton operator. The eigenvectors $\left\{u_{n}^{k}\right\}$ fulfilling the eigenvalue equation

$$
\sum_{n^{\prime} \in G} \mathcal{O}_{n, n^{\prime}} u_{n^{\prime}}^{k}=\varepsilon_{k}^{2} u_{n}^{k}
$$

can be written as a direct product:

$$
u_{n}^{k}=v_{n_{1}}^{k_{1}} w_{n_{2}}^{k_{2}} \cdots w_{n_{d}}^{k_{d}}=v_{n_{1}}^{k_{1}} w_{\vec{n}_{\perp}}^{\vec{k}_{\perp}},
$$

with

$$
k=\left(k_{1}, k_{2}, \ldots, k_{d}\right)=\left(k_{1}, \vec{k}_{\perp}\right) .
$$

The indices $k$ label the linearly independent vectors, of which there are exactly as many as there are points on the lattice - namely $\left(N_{1}-1\right)\left(2 N_{\perp}\right)^{d-1}$ which is also the dimenson of the real symmetric matrix $\mathcal{O}_{n, n^{\prime}}$. One can therefore choose the individual $k$ 's so they take the same values as the $n_{i}$ and consequently form the same lattice:

$$
G=\left\{k \in \mathbf{Z}^{d} \mid 1 \leq k_{1} \leq\left(N_{1}-1\right) ;\left(-N_{\perp}+1\right) \leq k_{i} \leq N_{\perp}, \quad i=2, \ldots d\right\} .
$$

Choosing the lattice as described above guarantees that the boundary conditions are automatically fulfilled.

The separation formula (2.44) leads to (compare eqs. (2.10) - 2.12))

$$
\begin{gathered}
\left.\sum_{n_{1}^{\prime}=1}^{N_{1}-1}\left[V\left(n_{1}\right) \delta_{n_{1}, n_{1}^{\prime}}-\frac{1}{a^{2}}\left(\delta_{n_{1}+1, n_{1}^{\prime}}-2 \delta_{n_{1}, n_{1}^{\prime}}+\delta_{n_{1}-1, n_{1}^{\prime}}\right)\right)\right] v_{n_{1}^{\prime}}^{k_{1}}=\rho_{k_{1}}^{2} v_{n_{1}}^{k_{1}}, \\
\sum_{n_{i}^{\prime}=-N_{\perp}+1}^{N_{\perp}}-\frac{1}{a^{2}}\left(\delta_{n_{i}+1, n_{i}^{\prime}}-2 \delta_{n_{i}, n_{i}^{\prime}}+\delta_{n_{i}-1, n_{i}^{\prime}}\right) w_{n_{i}^{\prime}}^{k_{i}}=\omega_{k_{i}}^{2} w_{n_{i}}^{k_{i}}, i=2, \cdots, d
\end{gathered}
$$


where $\rho_{k_{1}}^{2}, \omega_{k_{i}}^{2}$ are related to the eigenvalues $\varepsilon_{k}^{2}$ of (2.43) by $\varepsilon_{k}^{2}=m^{2}+\rho_{k}^{2}+\sum_{i=2}^{d} \omega_{k_{i}}^{2}$. While the $v_{n_{1}}^{k_{1}}$ can in general be obtained only numerically, the modes for the free directions can again be gotten analytically:

$$
\begin{aligned}
w_{n_{i}}^{k_{i}} & =\sqrt{\frac{1}{2 N_{\perp}}} e^{i \frac{\pi}{N_{\perp}} k_{i} n_{i}}, \\
\omega_{k_{i}}^{2} & =\frac{4}{a^{2}} \sin ^{2}\left(\frac{\pi k_{i}}{2 N_{\perp}}\right) .
\end{aligned}
$$

In summary

$$
\begin{aligned}
& u_{n}^{k}=v_{n_{1}}^{k_{1}} w_{\vec{n}_{\perp}}^{\vec{k}_{\perp}}=v_{n_{1}}^{k_{1}}\left(\frac{1}{2 N_{\perp}}\right)^{\frac{d-1}{2}} e^{i \frac{\pi}{N_{\perp}} \vec{k}_{\perp} \vec{n}_{\perp}}, \\
& \varepsilon_{k}^{2}=m^{2}+\rho_{k_{1}}^{2}+\sum_{i=2}^{d} \frac{4}{a^{2}} \sin ^{2}\left(\frac{\pi k_{i}}{2 N_{\perp}}\right) .
\end{aligned}
$$

These formulae are the lattice analogue of eqs. (2.16), (2.17) in the continuum problem with one little difference: the exact lattice analog of the orthogonality- and completeness relation (2.18) would be $\sum_{n \in G} a^{d} u_{n}^{k}\left(u_{n}^{k^{\prime}}\right)^{*}=\delta_{k, k^{\prime}}, \sum_{k \in G} u_{n}^{k}\left(u_{n}^{\prime k}\right)^{*}=$ $\delta_{n, n^{\prime}} / a^{d}$. In the limit $a \rightarrow 0$ these expressions tend to (2.18). But on the lattice it is more natural to work with the relations

$$
\begin{aligned}
\sum_{n \in G} u_{n}^{k}\left(u_{n}^{k^{\prime}}\right)^{*} & =\delta_{k, k^{\prime}}, \\
\sum_{k \in G} u_{n}^{k}\left(u_{n^{\prime}}^{k}\right)^{*} & =\delta_{n, n^{\prime}} .
\end{aligned}
$$

It is therefore the combination $u_{n}^{k} / \sqrt{a^{d}}$ which in the limit $a \rightarrow 0$ tends to $u^{k}(x)$. To diagonalize the Hamilton operator we make the following expansion of $\phi_{n}$ in terms of the eigenvectors $u_{n}^{k}$,

$$
\phi_{n}=\sum_{k \in G} \frac{u_{n}^{k}}{\sqrt{a^{d}}} \hat{\phi}_{k}
$$

with the inversion

$$
\hat{\phi}_{k}=\sum_{n \in G} \sqrt{a^{d}}\left(u_{n}^{k}\right)^{*} \phi_{n}=\sum_{n \in G} a^{d}\left(\frac{u_{n}^{k}}{\sqrt{a^{d}}}\right)^{*} \phi_{n} .
$$

Because the $\phi_{n}$ are real we have

$$
\hat{\phi}_{k}^{*}=\hat{\phi}_{k_{1}, \vec{k}_{\perp}}^{*}=\hat{\phi}_{k_{1},-\vec{k}_{\perp}} .
$$

Eqs. (2.53) and (2.54) imply for the derivatives

$$
\begin{aligned}
\frac{\partial}{\partial \phi_{n}} & =\sum_{k \in G} \sqrt{a^{d}}\left(u_{n}^{k}\right)^{*} \frac{\partial}{\partial \hat{\phi}_{k}}, \\
\frac{\partial}{\partial \hat{\phi}_{k}} & =\sum_{n \in G} \frac{1}{\sqrt{a^{d}}} u_{n}^{k} \frac{\partial}{\partial \phi_{n}} .
\end{aligned}
$$


In terms of the $\hat{\phi}_{k}$ the Hamilton operator (2.41) becomes

$$
\mathcal{H}=\frac{1}{2} \sum_{k \in G}\left(-\frac{\partial^{2}}{\partial \hat{\phi}_{k} \partial \hat{\phi}_{k}^{*}}+\varepsilon_{k}^{2} \hat{\phi}_{k} \hat{\phi}_{k}^{*}\right),
$$

a finite sum over uncoupled harmonic oscillator Hamiltonians. The remaining steps are familiar. We introduce annihilation/creation operators

$$
\begin{aligned}
& A_{k}=\frac{1}{\sqrt{2 \epsilon_{k}}}\left(\epsilon_{k} \hat{\phi}_{k}+\frac{\partial}{\partial \hat{\phi}_{k}^{*}}\right), \\
& A_{k}^{\dagger}=\frac{1}{\sqrt{2 \epsilon_{k}}}\left(\epsilon_{k} \hat{\phi}_{k}^{*}-\frac{\partial}{\partial \hat{\phi}_{k}}\right),
\end{aligned}
$$

with commutation relations $\left[A_{k}, A_{k^{\prime}}^{\dagger}\right]=\delta_{k k^{\prime}},\left[A_{k}, A_{k^{\prime}}\right]=\left[A_{k}^{\dagger}, A_{k^{\prime}}^{\dagger}\right]=0$, in terms of which the Hamilton operator (2.58) becomes

$$
\mathcal{H}=\frac{1}{2} \sum_{k \in G} \varepsilon_{k}\left(A_{k}^{\dagger} A_{k}+A_{k} A_{k}^{\dagger}\right)=\sum_{k \in G} \varepsilon_{k}\left(A_{k}^{\dagger} A_{k}+\frac{1}{2}\right) .
$$

One recognizes that one is dealing with a finite set of uncoupled harmonic oscillators whose frequencies are the spectrum $\left\{\epsilon_{k}\right\}$. A Fock space can now be constructed, whose ground state $|0\rangle$ is of course defined by $A_{k}|0\rangle=0 \forall k$. The (finite) energy of this state is

$$
E_{0}=\frac{1}{2} \sum_{k \in G} \varepsilon_{k}
$$

The representation of $\phi_{n}$ in terms of creation and annihilation operators is

$$
\phi_{n}=\sum_{k \in G} \frac{1}{\sqrt{2 \varepsilon_{k}}}\left(\frac{u_{n}^{k}}{\sqrt{a^{d}}} A_{k}+\frac{\left(u_{n}^{k}\right)^{*}}{\sqrt{a^{d}}} A_{k}^{\dagger}\right) .
$$

\subsubsection{Vacuum stress tensor}

To compute the vacuum expectation of the stress tensor operator (2.21) we recall that, in the Schrödinger picture and field representation, $\Pi(x, t)=\phi(x, t)=$ $-i \frac{\delta}{\delta \phi(x)}$. Thus

$$
\begin{aligned}
T^{00}(x) & =\frac{1}{2}\left(-\frac{\delta^{2}}{(\delta \phi)^{2}}+(\nabla \phi)^{2}+\left(m^{2}+V\left(x_{1}\right)\right) \phi^{2}\right) \\
& =\frac{1}{2}\left(-\frac{\delta^{2}}{(\delta \phi)^{2}}+\phi\left(m^{2}+V\left(x_{1}\right)-\triangle\right) \phi\right)+\frac{1}{2} \nabla(\phi \nabla \phi), \\
T^{i i}(x) & =\frac{1}{2}\left(-\frac{\delta^{2}}{(\delta \phi)^{2}}-\phi\left(m^{2}+V\left(x_{1}\right)-\triangle\right) \phi\right) \\
& -\frac{1}{2} \nabla(\phi \nabla \phi)+\left(\frac{\partial \phi}{\partial x_{i}}\right)^{2},
\end{aligned}
$$




$$
\begin{aligned}
T^{0 i}(x) & =-\frac{i}{2}\left(\frac{\partial \phi}{\partial x_{i}}\right) \frac{\delta}{\delta \phi}-\frac{i}{2} \frac{\delta}{\delta \phi}\left(\frac{\partial \phi}{\partial x_{i}}\right), \\
T^{i j}(x) & =\left(\frac{\partial \phi}{\partial x_{i}}\right)\left(\frac{\partial \phi}{\partial x_{j}}\right), \quad i \neq j .
\end{aligned}
$$

With the help of the correspondence (2.38) and eqs. (A.32), (A.33) one recasts (2.64), (2.65) in the form

$$
\begin{aligned}
T_{n}^{00}= & \frac{1}{2}\left(-\frac{1}{a^{2 d}} \frac{\partial^{2}}{\left(\partial \phi_{n}\right)^{2}}+\phi_{n} \sum_{n^{\prime} \in G} \mathcal{O}_{n, n^{\prime}} \phi_{n^{\prime}}\right)+\sum_{j=1}^{d} \frac{\phi_{n+\vec{e}_{j}}^{2}-2 \phi_{n}^{2}+\phi_{n-\vec{e}_{j}}^{2}}{4 a^{2}}, \\
T_{n}^{i i}= & \frac{1}{2}\left(-\frac{1}{a^{2 d}} \frac{\partial^{2}}{\left(\partial \phi_{n}\right)^{2}}-\phi_{n} \sum_{n^{\prime} \in G} \mathcal{O}_{n, n^{\prime}} \phi_{n^{\prime}}\right)-\sum_{j=1}^{d} \frac{\phi_{n+\vec{e}_{j}}^{2}-2 \phi_{n}^{2}+\phi_{n-\vec{e}_{j}}^{2}}{4 a^{2}} \\
& -\phi_{n} \frac{\phi_{n+\vec{e}_{i}}-2 \phi_{n}+\phi_{n-\vec{e}_{i}}}{a^{2}}+\frac{\phi_{n+\vec{e}_{i}}^{2}-2 \phi_{n}^{2}+\phi_{n-\vec{e}_{i}}^{2}}{2 a^{2}} .
\end{aligned}
$$

The next step is to take the vacuum expectation values of the operators (2.55), (2.56). For this purpose it is useful to note that

$$
\begin{aligned}
\hat{\phi}_{k} & =\hat{\phi}_{k_{1}, \vec{k}_{\perp}}=\frac{1}{\sqrt{2 \varepsilon_{k}}}\left(A_{k_{1}, \vec{k}_{\perp}}+A_{k_{1},-\vec{k}_{\perp}}^{\dagger}\right), \\
\frac{\partial}{\partial \hat{\phi}_{k}} & =\frac{\partial}{\partial \hat{\phi}_{k_{1}, \vec{k}_{\perp}}}=\sqrt{\frac{\varepsilon_{k}}{2}}\left(A_{k_{1},-\vec{k}_{\perp}}-A_{k_{1}, \vec{k}_{\perp}}^{\dagger}\right) .
\end{aligned}
$$

One then verifies

$$
\begin{aligned}
\left\langle 0\left|\hat{\phi}_{k} \hat{\phi}_{k^{\prime}}^{*}\right| 0\right\rangle & =\frac{1}{2 \varepsilon_{k}} \delta_{k, k^{\prime}}, \\
\left\langle 0\left|\frac{\partial^{2}}{\partial \hat{\phi}_{k} \partial \hat{\phi}_{k^{\prime}}^{*}}\right| 0\right\rangle & =-\frac{1}{2} \varepsilon_{k} \delta_{k, k^{\prime}}, \\
\left\langle 0\left|\frac{\partial}{\partial \hat{\phi}_{k}} \hat{\phi}_{k^{\prime}}\right| 0\right\rangle=-\left\langle 0\left|\hat{\phi}_{k} \frac{\partial}{\partial \hat{\phi}_{k^{\prime}}}\right| 0\right\rangle & =\frac{1}{2} \delta_{k, k^{\prime}},
\end{aligned}
$$

and

$$
\begin{aligned}
\left\langle 0\left|-\frac{\partial^{2}}{a^{d}\left(\partial \phi_{n}\right)^{2}}\right| 0\right\rangle & =\left\langle 0\left|a^{d} \phi_{n} \sum_{n^{\prime} \in G} \mathcal{O}_{n, n^{\prime}} \phi_{n^{\prime}}\right| 0\right\rangle \\
& =\frac{1}{2} \sum_{k \in G} \varepsilon_{k}\left|u_{n}^{k}\right|^{2} .
\end{aligned}
$$

Using (2.46), (2.47) and the completeness relations for the eigenvectors one finds

$$
\left\langle 0\left|T_{n}^{00}\right| 0\right\rangle=\sum_{k \in G} \frac{\varepsilon_{k}}{2 a^{d}}\left|u_{n}^{k}\right|^{2}+\sum_{k \in G} \sum_{j=1}^{d} \frac{1}{8 a^{d+2} \varepsilon_{k}}\left(\left|u_{n+\vec{e}_{j}}^{k}\right|^{2}-2\left|u_{n}^{k}\right|^{2}+\left|u_{n-\vec{e}_{j}}^{k}\right|^{2}\right)
$$




$$
\begin{gathered}
\left\langle 0\left|T_{n}^{11}\right| 0\right\rangle=\sum_{k} \frac{\rho_{k_{1}}^{2}-V\left(n_{1}\right)}{2 a^{d} \varepsilon_{k}}\left|u_{n}^{k}\right|^{2}+\sum_{k \in G} \frac{1}{8 a^{d+2} \varepsilon_{k}}\left(\left|u_{n+\vec{e}_{1}}^{k}\right|^{2}-2\left|u_{n}^{k}\right|^{2}+\left|u_{n-\vec{e}_{1}}^{k}\right|^{2}\right) \\
-\sum_{k \in G} \sum_{j=2}^{d} \frac{1}{8 a^{d+2} \varepsilon_{k}}\left(\left|u_{n+\vec{e}_{j}}^{k}\right|^{2}-2\left|u_{n}^{k}\right|^{2}+\left|u_{n-\vec{e}_{j}}^{k}\right|^{2}\right)
\end{gathered}
$$

and for $i=2, \cdots, d$

$$
\begin{array}{r}
\left\langle 0\left|T_{n}^{i i}\right| 0\right\rangle=\sum_{k} \frac{\omega_{k_{i}}^{2}}{2 a^{d} \varepsilon_{k}}\left|u_{n}^{k}\right|^{2}+\sum_{k \in G} \frac{1}{8 a^{d+2} \varepsilon_{k}}\left(\left|u_{n+\vec{e}_{i}}^{k}\right|^{2}-2\left|u_{n}^{k}\right|^{2}+\left|u_{n-\vec{e}_{i}}^{k}\right|^{2}\right) \\
-\sum_{k \in G} \sum_{\substack{j=1 \\
j \neq i}}^{d} \frac{1}{8 a^{d+2} \varepsilon_{k}}\left(\left|u_{n+\vec{e}_{j}}^{k}\right|^{2}-2\left|u_{n}^{k}\right|^{2}+\left|u_{n-\vec{e}_{j}}^{k}\right|^{2}\right) .
\end{array}
$$

Finally from eq. (2.49)

$$
\left|u_{n}^{k}\right|^{2}=\frac{\left(v_{n_{1}}^{k_{1}}\right)^{2}}{\left(2 N_{\perp}\right)^{d-1}}
$$

so the preceding formulae can be simplified. Thus with $L=a N_{\perp}$

$$
\begin{aligned}
\left\langle 0\left|T_{n}^{00}\right| 0\right\rangle & =\sum_{k \in G} \frac{\varepsilon_{k}\left(v_{n_{1}}^{k_{1}}\right)^{2}}{2 a(2 L)^{d-1}}+\sum_{k \in G} \frac{\left(v_{n_{1}+1}^{k_{1}}\right)^{2}-2\left(v_{n_{1}}^{k_{1}}\right)^{2}+\left(v_{n_{1}-1}^{k_{1}}\right)^{2}}{8 a^{3} \varepsilon_{k}(2 L)^{d-1}} \\
\left\langle 0\left|T_{n}^{11}\right| 0\right\rangle & =\sum_{k \in G} \frac{\left(\rho_{k_{1}}^{2}-V\left(n_{1}\right)\right)\left(v_{n_{1}}^{k_{1}}\right)^{2}}{2 a \varepsilon_{k}(2 L)^{d-1}} \\
& +\sum_{k \in G} \frac{\left(v_{n_{1}+1}^{k_{1}}\right)^{2}-2\left(v_{n_{1}}^{k_{1}}\right)^{2}+\left(v_{n_{1}-1}^{k_{1}}\right)^{2}}{8 a^{3} \varepsilon_{k}(2 L)^{d-1}}, \\
\left\langle 0\left|T_{n}^{i i}\right| 0\right\rangle & =\sum_{k \in G} \frac{\omega_{k_{i}}^{2}\left(v_{n_{1}}^{k_{1}}\right)^{2}}{2 a \varepsilon_{k}(2 L)^{d-1}} \\
& -\sum_{k \in G} \frac{\left(v_{n_{1}+1}^{k_{1}}\right)^{2}-2\left(v_{n_{1}}^{k_{1}}\right)^{2}+\left(v_{n_{1}-1}^{k_{1}}\right)^{2}}{8 a^{3} \varepsilon_{k}(2 L)^{d-1}}, \quad i \geq 2 .
\end{aligned}
$$

Eqs. (2.76) - (2.78) are the lattice analogues of eqs. (2.22) - (2.24) in the continuum problem. One can transform the latter directly to the lattice with the help of eqs. (A.32), (A.33) and the correspondence

$$
u^{k}(x) \rightarrow \frac{u_{n}^{k}}{\sqrt{a^{d}}} \quad, \quad\left(v^{k_{1}}\left(x_{1}\right)\right)^{2} \rightarrow \frac{\left(v_{n_{1}}^{k_{1}}\right)^{2}}{a} .
$$

Eqs. (2.76) - (2.78) are valid on the lattice $n \in G$. However they do not hold for the Dirichlet boundary surfaces $n_{1}=0, N_{1}$ which have to be dealt with separately. On these surfaces one must use the unsymmetric derivative formula discussed in Appendix A which ensures a smooth continuation of the lattice mathematics onto the Dirichlet boundary surfaces. 
The calculation of $\left\langle T^{\mu \nu}\right\rangle$ on the Dirichlet surfaces using the one-sided derivative is equivalent (see eqs. (A.28), (A.29) ) to introducing in eqs. (2.76) - (2.78) the additional definitions

$$
\begin{aligned}
\left(v_{-1}^{k_{1}}\right)^{2} & :=\left(v_{1}^{k_{1}}\right)^{2}, \\
\left(v_{N_{1}+1}^{k_{1}}\right)^{2} & :=\left(v_{N_{1}-1}^{k_{1}}\right)^{2} .
\end{aligned}
$$

Thus on the Dirichlet boundary surfaces

$$
\begin{aligned}
\left\langle 0\left|T_{0, \vec{n}_{\perp}}^{00}\right| 0\right\rangle & =\left\langle 0\left|T_{0, \vec{n}_{\perp}}^{11}\right| 0\right\rangle=\sum_{k \in G} \frac{\left(v_{1}^{k_{1}}\right)^{2}}{4 a^{3} \varepsilon_{k}(2 L)^{d-1}}=-\left\langle 0\left|T_{0, \vec{n}_{\perp}}^{i i}\right| 0\right\rangle, \\
\left\langle 0\left|T_{N_{1}, \vec{n}_{\perp}}^{00}\right| 0\right\rangle & =\left\langle 0\left|T_{N_{1}, \vec{n}_{\perp}}^{11}\right| 0\right\rangle=\sum_{k \in G} \frac{\left(v_{N_{1}-1}^{k_{1}}\right)^{2}}{4 a^{3} \varepsilon_{k}(2 L)^{d-1}}=-\left\langle 0\left|T_{N_{1}, \vec{n}_{\perp}}^{i i}\right| 0\right\rangle .
\end{aligned}
$$

We see that the unregularized $\left\langle T^{00}\right\rangle$ and $\left\langle T^{11}\right\rangle$ are the same on the boundary surfaces. However, the renormalizations of $\left\langle T^{00}\right\rangle$ and $\left\langle T^{11}\right\rangle$ turn out to be different (secs. 3 and 4). Thus the renormalized tensor components $\left\langle T^{00}\right\rangle_{\text {ren }}$ and $\left\langle T^{11}\right\rangle_{\text {ren }}$ on the boundary surfaces end up being different.

Using the lattice definition (A.31) of "spatial integration" along $x_{1}$ we find

$$
\frac{1}{2}\left(\sum_{n_{1}=1}^{N_{1}}+\sum_{n_{1}=0}^{N_{1}-1}\right) \sum_{\vec{n}_{\perp}}\left\langle 0\left|T_{n}^{00}\right| 0\right\rangle a^{d}=\sum_{k \in G} \frac{1}{2} \varepsilon_{k}=E_{0} .
$$

This relation must hold, of course, for $\left\langle T_{n}^{00}\right\rangle$ to be the unrenormalized lattice vacuum energy density.

\subsubsection{The case $V\left(n_{1}\right)=0$}

When the lattice potential $V\left(n_{1}\right)=0$ between the Dirichlet walls $n_{1}=0, N_{1}$ one can easily solve the lattice Schrödinger mode equation (2.46). The eigenvectors and eigenvalues are

$$
\begin{aligned}
v_{n_{1}}^{k_{1}} & =\sqrt{\frac{2}{N_{1}}} \sin \left(\frac{\pi}{N_{1}} k_{1} n_{1}\right), \\
\rho_{k_{1}}^{2} & =\frac{4}{a^{2}} \sin ^{2}\left(\frac{\pi k_{1}}{2 N_{1}}\right), \quad k_{1}=1,2,3, \ldots, N_{1} .
\end{aligned}
$$

Thus

$$
\begin{aligned}
u_{n}^{k} & =\sqrt{\frac{2}{N_{1}}} \sin \left(\frac{\pi k_{1}}{N_{1}} n_{1}\right)\left(\frac{1}{2 N_{\perp}}\right)^{\frac{d-1}{2}} e^{i \frac{\pi}{N_{\perp}} \vec{k}_{\perp} \vec{n}_{\perp}}, \\
\varepsilon_{k}^{2} & =m^{2}+\frac{4}{a^{2}} \sin ^{2}\left(\frac{\pi k_{1}}{2 N_{1}}\right)+\sum_{i=2}^{d} \frac{4}{a^{2}} \sin ^{2}\left(\frac{\pi k_{i}}{2 N_{\perp}}\right) .
\end{aligned}
$$


The lattice vacuum energy is

$$
E_{0}=\frac{1}{2} \sum_{k \in G} \varepsilon_{k}=\frac{1}{2} \sum_{k \in G} \sqrt{m^{2}+\frac{4}{a^{2}} \sin ^{2}\left(\frac{\pi k_{1}}{2 N_{1}}\right)+\sum_{i=2}^{d} \frac{4}{a^{2}} \sin ^{2}\left(\frac{\pi k_{i}}{2 N_{\perp}}\right)} .
$$

In computing the vacuum stress tensor we make use of the identities

$$
\begin{aligned}
\sin ^{2}(\alpha(n+1))-2 \sin ^{2}(\alpha n)+\sin ^{2}(\alpha(n-1)) & =2 \sin ^{2}(\alpha) \cos (2 \alpha n) \quad, \quad(2.88) \\
\sin ^{2}(\alpha) & =4 \sin ^{2}\left(\frac{\alpha}{2}\right)\left(1-\sin ^{2}\left(\frac{\alpha}{2}\right)\right) .
\end{aligned}
$$

Thus

$$
\begin{aligned}
& \sin ^{2}\left(\frac{\pi k_{1}}{N_{1}}\left(n_{1}+1\right)\right)-2 \sin ^{2}\left(\frac{\pi k_{1}}{N_{1}} n_{1}\right)+\sin ^{2}\left(\frac{\pi k_{1}}{N_{1}}\left(n_{1}-1\right)\right)= \\
& 2 a^{2} \rho_{k_{1}}^{2} \cos \left(\frac{2 \pi k_{1} n_{1}}{N_{1}}\right)-2 a^{2} \rho_{k_{1}}^{2} \sin ^{2}\left(\frac{\pi k_{1}}{2 N_{1}}\right) \cos \left(\frac{2 \pi k_{1} n_{1}}{N_{1}}\right) .
\end{aligned}
$$

Then from eqs. (2.76) - (2.78) and with $l=N_{1} / a$

$$
\begin{aligned}
\left\langle 0\left|T_{n}^{00}\right| 0\right\rangle= & \frac{1}{l(2 L)^{d-1}} \sum_{k \in G} \frac{1}{2} \varepsilon_{k}-\sum_{k \in G} \frac{\left(\varepsilon_{k}^{2}-\rho_{k_{1}}^{2}\right)}{2 l(2 L)^{d-1} \varepsilon_{k}} \cos \left(\frac{2 \pi k_{1} n_{1}}{N_{1}}\right) \\
& -\sum_{k \in G} \frac{\rho_{k_{1}}^{2}}{2 l(2 L)^{d-1} \varepsilon_{k}} \sin ^{2}\left(\frac{\pi k_{1}}{2 N_{1}}\right) \cos \left(\frac{2 \pi k_{1} n_{1}}{N_{1}}\right), \\
\left\langle 0\left|T_{n}^{11}\right| 0\right\rangle= & \sum_{k \in G} \frac{\rho_{k_{1}}^{2}}{2 l(2 L)^{d-1} \varepsilon_{k}} \\
- & \sum_{k \in G} \frac{\rho_{k_{1}}^{2}}{2 l(2 L)^{d-1} \varepsilon_{k}} \sin ^{2}\left(\frac{\pi k_{1}}{2 N_{1}}\right) \cos \left(\frac{2 \pi k_{1} n_{1}}{N_{1}}\right), \\
\left\langle 0\left|T_{n}^{i i}\right| 0\right\rangle= & \frac{1}{l(2 L)^{d-1}} \sum_{k \in G} \frac{1}{2} \omega_{k_{i}}^{2}-\sum_{k \in G} \frac{\rho_{k_{1}}^{2}+\omega_{k_{i}}^{2}}{2 l(2 L)^{d-1} \varepsilon_{k}} \cos \left(\frac{2 \pi k_{1} n_{1}}{N_{1}}\right) \\
& +\sum_{k \in G} \frac{\rho_{k_{1}}^{2}}{2 l(2 L)^{d-1} \varepsilon_{k}} \sin ^{2}\left(\frac{\pi k_{1}}{2 N_{1}}\right) \cos \left(\frac{2 \pi k_{1} n_{1}}{N_{1}}\right), \quad i \geq 2 .
\end{aligned}
$$

Comparing eqs. (2.90) - (2.92) and the continuum formulae (2.22) - (2.24) with background potential $V\left(x_{1}\right)=0$ and $v^{k_{1}}\left(x_{1}\right)=\sqrt{2 / l} \sin \left(\pi k_{1} x_{1} / l\right), k_{1}=1,2,3, \cdots$ one finds in the former the presence of a lattice artefact term

$$
A \equiv \sum_{k \in G} \frac{2 \sin ^{4}\left(\frac{\pi k_{1}}{2 N_{1}}\right) \cos \left(\frac{2 \pi k_{1} n_{1}}{N_{1}}\right)}{a^{2} l(2 L)^{d-1} \varepsilon_{k}} .
$$

This term has no continuum counterpart and it diverges for $a \rightarrow 0$. In Appendix $\mathrm{B}$ we investigate the $1 \mathrm{D}$ version of $A$ in considerable detail and conclude that it is essentially devoid of physical information. 


\section{One dimensional lattice without background po- tential}

In this and the following section we specialize to one-dimensional lattice systems with no additional free directions $x_{2}, \cdots, x_{d}$. We drop the subscript 1 and first consider the case of vanishing potential $V(x)$. In sec. 4 we will introduce a specific nonzero potential along the lattice. In both sections we wish to deal with Casimir forces. This means we must also consider one or more regions external to the interval $0<x<l$ or $0<n<N=l / a$. This is easily done. We can attach external regions which are exactly like the internal region $0<x<l$ (except for their length) with $V(x)=0$ and a Dirichlet boundary at either end. When we calculate the lattice vacuum energy $E_{v a c}(l)$ in $0<x<l$ the same calculation gives us the lattice vacuum energy $E_{v a c}(L-l)$ in the adjacent interval $l<x<L$. Likewise one calculation gives us the lattice $T^{\mu \nu}$ in both intervals. Our principal goals here are to understand in detail how to renormalize $E_{v a c}$ and $T^{\mu \nu}$ in the continuum limit $a \rightarrow 0$. Then we can calculate the Casimir force on the common Dirichlet boundary $x=l$ in two ways: globally using $E_{v a c}$ and locally using $T^{11}$. Comparison with the continuum version of this system will be important. We shall find that in every respect the lattice theory closely approximates the continuum system as expected. It will be convenient to discuss the cases of zero and nonzero field mass separately.

\subsection{Renormalized vacuum energy}

\subsubsection{Zero mass}

We find from eq. (2.87) the lattice vacuum energy in the internal region $0 \leq x \leq l$ :

$$
E_{r e g}(l, a)=\frac{1}{2} \sum_{k=1}^{N-1} \rho_{k}=\frac{1}{a} \sum_{k=1}^{N-1} \sin \left(\frac{\pi k}{2 N}\right)
$$

where $N=l / a$. The label "reg" here (and subsequently) is used to denote lattice quantities. The finite sum (3.1) can be evaluated using [7]

$$
\sum_{k=1}^{N-1} \sin (k x)=\sin \left(\frac{N x}{2}\right) \sin \left(\frac{(N-1) x}{2}\right) \csc \left(\frac{x}{2}\right)
$$

with the result

$$
E_{\text {reg }}(l, a)=\frac{1}{2 a}\left(\frac{1}{\tan \left(\frac{\pi a}{4 l}\right)}-1\right) .
$$

Expanding in powers of $a$ yields

$$
E_{r e g}(l, a)=\frac{2 l}{\pi a^{2}}-\frac{1}{2 a}-\frac{\pi}{24 l}+\mathcal{O}\left(a^{2}\right) .
$$


Subtracting all terms in eq. (3.3) which diverge in the continuum limit $a \rightarrow 0$ we recover the continuum Casimir energy for a real 1D massless scalar field confined between Dirichlet boundaries [8, 9, 10],

$$
E(l)=\lim _{a \rightarrow 0}\left(E_{\text {reg }}(l, a)-\frac{2 l}{\pi a^{2}}+\frac{1}{2 a}\right)=-\frac{\pi}{24 l} .
$$

To directly derive $E(l)$ from the lattice energy (3.1) one can also do the following:

$$
\begin{aligned}
\lim _{N \rightarrow \infty}\left[\sum_{k=1}^{N-1}\left(\frac{1}{N \sin \left(\frac{\pi k}{2 N}\right)}\right)^{s}\right] & =\left(\frac{2}{\pi}\right)^{s} \sum_{k=1}^{\infty}\left(\frac{1}{k}\right)^{s} \\
& =\left(\frac{2}{\pi}\right)^{s} \zeta(s), \quad \operatorname{Re}(\mathrm{s})>1
\end{aligned}
$$

where $\zeta(s)$ is the Riemann $\zeta$ function [1]. Because $\zeta(s)$ can be continued throughout the $s$-plane (and is a meromorphic function with its only pole at $s=1$ ) one can use the preceding formula to evaluate the $N \rightarrow \infty$ limit of eq. (3.1):

$$
\begin{aligned}
\lim _{N \rightarrow \infty} E_{r e g}(l, a) & =\lim _{N \rightarrow \infty} \frac{1}{a N} \sum_{k=1}^{N-1} N \sin \frac{\pi k}{2 N} \\
& =\frac{1}{l} \frac{\pi}{2} \zeta(-1)=-\frac{\pi}{24 l} .
\end{aligned}
$$

To have a more complete physical system we add the external region $l \leq x \leq L$. Exactly the same calculations with $l$ replaced by $L-l$ give the lattice and continuum vacuum energies in this region; these are $E_{r e g}(L-l, a)$ and $E(L-l)$ respectively. The total lattice and continuum vacuum energies in $0 \leq x \leq L$ are then

$$
\begin{aligned}
E(l, L, a) & \equiv E_{r e g}(l, a)+E_{r e g}(L-l, a), \\
E(l, L) & \equiv E(l)+E(L-l) .
\end{aligned}
$$

Similarly an external region $-L^{\prime} \leq x \leq 0$ could be attached to the other side of the interval $[0, l]$. Additional external regions beyond these could be attached. Global structure on $x$ could also be imposed; e.g. periodicity on some large interval, or noncompactness for $x \rightarrow \pm \infty$. We shall do none of these things here. As stated, our sole purpose will be to probe the closeness of the lattice and continuum descriptions in $0 \leq x \leq L$.

Consider for example the global Casimir force on the Dirichlet boundary at $x=l$. The continuum definition of this force (in the direction of positive $x$ ) is

$$
\begin{aligned}
F_{C a s}(l, L) & =-\frac{\partial}{\partial l} E(l, L) \\
& =-\frac{\pi}{24 l^{2}}+\frac{\pi}{24(L-l)^{2}} .
\end{aligned}
$$


On the lattice one would define

$$
F_{C a s}(l, L, a)=-\frac{E_{r e g}(l+a, a)+E_{r e g}(L-(l+a), a)-E_{r e g}(l, a)-E_{r e g}(L-l, a)}{a} .
$$

In the limit $a \rightarrow 0$ and using eq. (3.3) one verifies that the same Casimir force (3.9) is recovered without the need to perform explicit subtractions.

\subsubsection{Nonzero mass}

For nonzero mass the lattice vacuum energy in the internal region $0 \leq x \leq l$

$$
\begin{aligned}
E_{r e g}(m, l, a) & =\frac{1}{2} \sum_{k=1}^{N-1} \sqrt{m^{2}+\frac{4}{a^{2}} \sin ^{2}\left(\frac{\pi k}{2 N}\right)} \\
& =\frac{1}{2 l} \sum_{k=1}^{N-1} \sqrt{(m l)^{2}+4 N^{2} \sin ^{2}\left(\frac{\pi k}{2 N}\right)}
\end{aligned}
$$

evidently cannot be evaluated in closed form. It can, however, usefully be expanded in powers of $m l$ :

$$
\begin{aligned}
E_{r e g}(m, l, a)= & \frac{N}{l} \sum_{k=1}^{N-1} \sin \left(\frac{\pi k}{2 N}\right)+\frac{1}{8} m^{2} l \sum_{k=1}^{N-1} \frac{1}{N \sin \left(\frac{\pi k}{2 N}\right)} \\
& +\frac{1}{l} \sum_{\nu=2}^{\infty} c_{\nu}\left(\frac{m l}{2}\right)^{2 \nu} \sum_{k=1}^{N-1}\left(\frac{1}{N \sin \left(\frac{\pi k}{2 N}\right)}\right)^{2 \nu-1}, \\
c_{\nu}= & \left(\begin{array}{c}
1 / 2 \\
\nu
\end{array}\right)=\frac{\Gamma(3 / 2)}{\nu ! \Gamma(3 / 2-\nu)} .
\end{aligned}
$$

The first term in eq. (3.12) is just the $m=0$ energy (3.1) which we already know how to renormalize. All of the terms in eq. (3.12) labeled by $\nu \geq 2$ are convergent in the continuum limit. Each of them can, in this limit, be expressed in terms of the Riemann $\zeta$ function using eq. (3.6) with $s$ values $s=2 \nu-1=3,5, \cdots$ for which this latter series converges. Hence they require no renormalization. However the second term in eq. (3.12) does require renormalization. This is evident from eq. (3.6) where evaluation of the term in question corresponds to using $\zeta(s)$ at its pole $s=1$. More directly, the second term in eq. (3.12) diverges logarithmically for $N \rightarrow \infty$. Indeed for $N \gg k$

$$
N \sin \left(\frac{\pi k}{2 N}\right) \approx \frac{\pi k}{2}
$$

and it is well known that

$$
\lim _{N \rightarrow \infty}\left(\sum_{k=1}^{N} \frac{1}{k}-\ln N\right) \equiv \gamma=0.5772 \ldots
$$


is a definition of Euler's constant $\gamma$. Analogously we define here

$$
\begin{aligned}
\sum_{k=1}^{N-1} \frac{1}{N \sin \left(\frac{\pi k}{2 N}\right)} & =\frac{2}{\pi} \ln N+c(N), \\
\lim _{N \rightarrow \infty}(c(N)) & =0.52125 \ldots
\end{aligned}
$$

which specifies another sequence $\{c(N)\}$ whose limiting value for $N \rightarrow \infty$ can be obtained numerically with the result shown in eq. (3.15). Thus the second term on the right of eq. (3.12) behaves as

$$
-\frac{1}{4 \pi} m^{2} l \ln \left(\frac{a}{l}\right)+\frac{m^{2} l}{8} c(N)
$$

i.e. there is an additional logarithmic divergence which has to be subtracted. By subtracting from $E_{\text {reg }}(m, l, a)$ the first term of (3.16) as it stands, we would incorrectly discard the $l$-dependence of this term. We must therefore split this term into the finite term $\frac{1}{4 \pi} m^{2} l \ln (\mu l)$ and a term $-\frac{1}{4 \pi} m^{2} l \ln (\mu a)$ diverging for $a \rightarrow 0$. Here $\mu$ is an arbitrary constant with dimension [mass] which has to be introduced to make the arguments of the logarithms dimensionless. We denote by $E(m, l, a, \mu)$ the expression obtained by subtracting this latter term, in addition to the divergent terms found in (3.3), from $E_{\text {reg }}(m, l, a)$, i.e.

$$
E(m, l, a, \mu) \equiv E_{r e g}(m, l, a)-\frac{2 l}{\pi a^{2}}+\frac{1}{2 a}+\frac{m^{2} l}{4 \pi} \ln (\mu a) .
$$

$E(m, l, a, \mu)$ converges in the limit $a \rightarrow 0$. The renormalized continuum vacuum energy in the internal region $0 \leq x \leq l$ can therefore be defined to be

$$
E(m, l, \mu):=\lim _{a \rightarrow 0} E(m, l, a, \mu) .
$$

All the preceding global formulae can be transferred to the external region $l \leq$ $x \leq L$ by simply replacing $l$ with $L-l$. The total vacuum energy for the system comprising the region $0 \leq x \leq L$ with Dirichlet boundaries at $x=0, l$ and $L$ is $E(m, l, a, \mu)+E(m, L-l, a, \mu)$. The Casimir force on the boundary point at $x=l$ is then

$$
\begin{aligned}
F_{C a s}(m, l, L)=-\lim _{a \rightarrow 0} \frac{1}{a}[E(m, l+a, a, \mu) & +E(m, L-l-a, a, \mu) \\
& -E(m, l, a, \mu)-E(m, L-l, a, \mu)] .
\end{aligned}
$$

One can easily convince oneself that the divergences which have to be subtracted in order to make the renormalized continuum vacuum energy $E(m, l, \mu)$ finite, cancel in the difference on the right of eq. (3.19) in the limit $a \rightarrow 0$. I. e. in the lattice calculation of $F_{C a s}$ these terms do not need to be subtracted from $E_{\text {reg }}$ and the measurable Casimir force, unlike the vacuum energy, does not depend on the arbitrary 
parameter $\mu$.

Physically fixing $\mu$

The dependence of the $m>0$ renormalized continuum vacuum energy $E(m, l, \mu)$ on the arbitrary mass parameter $\mu$ seems unsatisfying. This does not, of course, mean that it is wrong. A vacuum energy in QFT evidently need not be absolutely calculable. (However shifts in vacuum energies - i.e. Casimir energies and their associated Casimir forces - should be absolutely calculable as is the case here.) We now propose a way to select a particular value of $\mu$ which, while not dictated by general theory, is physically motivated.

To this end let us make the ansatz

$$
\mu=\kappa m
$$

where $\kappa$ is a dimensionless proportionality constant which will be determined by the requirement

$$
\lim _{l \rightarrow \infty}\left(-\frac{\partial E(m, l, \kappa m)}{\partial l}\right)=0
$$

This means that the contribution to the Casimir force on the boundary $x=l$ is determined by $-\frac{\partial}{\partial l} E(m, l, \mu)$ alone if $L$ is chosen to be $\infty$. One can verify that for $\mu=m$

$$
\lim _{l \rightarrow \infty} E(m, l, \mu=m)=-\frac{m^{2} l}{4 \pi} \ln (\chi)-\frac{m}{4},
$$

where $\chi$ is a specific constant which can be determined numerically with the result $\ln \chi=-2.579436$. Using this $\chi$ we define a new renormalized continuum vacuum energy by

$$
\begin{aligned}
E(m, l) & \equiv E(m, l, \mu=m)+\frac{m^{2} l}{4 \pi} \ln \chi \\
& =\lim _{a \rightarrow 0}\left(E_{r e g}(m, l, a)-\frac{2 l}{\pi a^{2}}+\frac{1}{2 a}+\frac{m^{2} l}{4 \pi} \ln (\chi m a)\right)
\end{aligned}
$$

which has the large- $l$ behavior

$$
\lim _{l \rightarrow \infty} E(m, l)=-\frac{m}{4} .
$$

Hence, if we identify the parameter $\kappa$ introduced above with the numerically determined constant $\chi$, this vacuum energy $E(m, l)$ achieves the goal set in eq. (3.21). Let us give a bit more detail. From eqs. (3.5), (3.11) - (3.15)

$$
E(m, l)=-\frac{\pi}{24 l}+\frac{1}{4 \pi} m^{2} l \ln (\tilde{\chi} m l)
$$




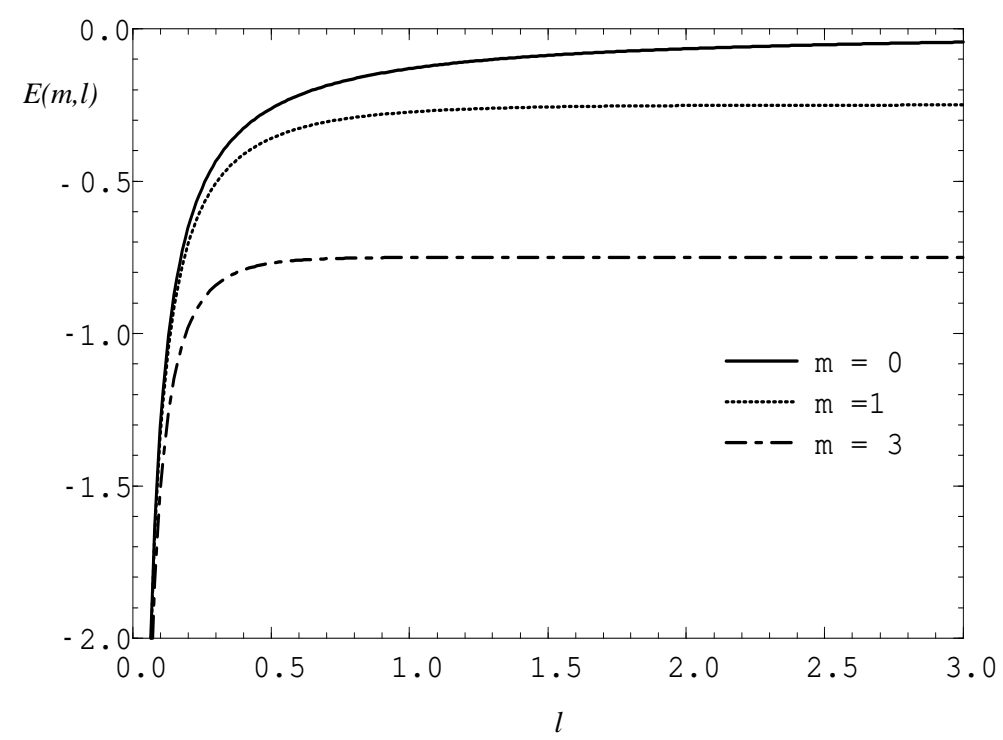

Figure 1: $E(m, l)$ from (3.23) with $\chi=0.0758166 . \quad E(m, l)$ is summed to $k=1000$.

In this and the following figures an arbitrary length unit is used.

$$
\begin{aligned}
& +\frac{1}{2 l} \lim _{N \rightarrow \infty}\left[\sum_{k=1}^{N-1}\left(\sqrt{(m l)^{2}+4 N^{2} \sin ^{2}\left(\frac{\pi k}{2 N}\right)}-2 N \sin \left(\frac{\pi k}{2 N}\right)-\frac{(m l)^{2}}{4 N \sin \left(\frac{\pi k}{2 N}\right)}\right)\right] \\
& =-\frac{\pi}{24 l}+\frac{1}{4 \pi} m^{2} l \ln (\tilde{\chi} m l)+\frac{1}{2 l} \sum_{k=1}^{\infty}\left(\sqrt{(m l)^{2}+(\pi k)^{2}}-\pi k-\frac{(m l)^{2}}{2 \pi k}\right)
\end{aligned}
$$

where $\ln (\tilde{\chi})$ differs from $\ln (\chi)$ by $\pi c(\infty) / 2, c(\infty)$ being the constant introduced in (3.15):

$$
\ln (\tilde{\chi})=\ln (\chi)+\frac{\pi}{2} 0.52125 \ldots=-1.76066 \ldots
$$

In fig. 1 we plot $E(m, l)$ versus $l$ for three different masses. An arbitrary length unit is involved in Fig. 1. The relevant scaling property of $E(m, l)$ is obvious in (3.25). In natural units $(\hbar=c=1)$ the only dimensional quantities available are $l$ and $m$, with $m l$ dimensionless. Thus $l$ sets the scale in (3.25). $E(m, l)$ is negative, increasingly so for increasing $m$ and fixed $l$. For fixed $m$ and increasing $l$ we see that $E(m, l)$ increases, corresponding to an attractive Casimir force $F_{C a s}$ between the boundaries at $x=0$ and $x=l$, where due to our fixing of $\mu$ no account has to be taken of the external regions $x<0, x>l$ provided they are chosen to extend to infinity. In this case the Casimir force on the boundaries at $x=0, l$ becomes

$$
\begin{aligned}
F_{C a s}(m, l) & =\lim _{a \rightarrow 0}\left(\frac{2}{a^{2}} \sum_{k=1}^{N-1} \frac{\sin ^{2}\left(\frac{\pi k}{2 N}\right)}{\sqrt{(m l)^{2}+4 N^{2} \sin ^{2}\left(\frac{\pi k}{2 N}\right)}}\right. \\
& \left.-\frac{2}{\pi a^{2}}+\frac{1}{2 a l}-\frac{m^{2}}{4 \pi} \ln (\chi m a)-\frac{m^{2}}{4 \pi}\right)
\end{aligned}
$$




$$
\begin{aligned}
& =-\frac{\pi}{24 l^{2}}-\frac{m^{2}}{4 \pi}(\ln (\tilde{\chi} m l)+1) \\
& +\frac{1}{2 l^{2}} \sum_{k=1}^{\infty}\left(\frac{(\pi k)^{2}}{\sqrt{(m l)^{2}+(\pi k)^{2}}}-\pi k+\frac{(m l)^{2}}{2 \pi k}\right) .
\end{aligned}
$$

Comparison with analytic results

Many authors have calculated the vacuum energy shift of the massless and massive 1D scalar field confined between Dirichlet points. An early and useful analysis in arbitrary dimension was given by Ambjørn and Wolfram [9]. We simply quote their result for $\mathrm{d}=1$ :

$$
\begin{aligned}
E_{A W}(m=0, l) & =-\frac{\pi}{24 l}, \\
E_{A W}(m, l) & =-\frac{m}{2 \pi} \sum_{n=1}^{\infty} \frac{K_{1}(2 m l n)}{n}, \\
\lim _{l \rightarrow \infty} E_{A W}(m, l) & =0,
\end{aligned}
$$

where $K_{1}(z)$ is a modified Bessel function. Note that no arbitrary mass parameter $\mu$ appears in $E_{A W}(m, l)$ and that $\lim _{l \rightarrow \infty} E_{A W}(m, l)=0$, i.e. the situation is essentially the same as above after fixing $\mu$ by the requirement $\frac{\partial}{\partial l} E(m, l)=0$, the only difference being that our $E(m, l)$ tends to $-m / 4$ for $l \rightarrow \infty$ whereas $\lim _{l \rightarrow \infty} E_{A W}(m, l)=0$. Let us form the ratio

$$
e_{A W}(m l) \equiv \frac{E_{A W}(m, l)}{E_{A W}(m=0, l)}=\frac{12 m l}{\pi^{2}} \sum_{n=1}^{\infty} \frac{K_{1}(2 m l n)}{n}
$$

and the corresponding ratio using our result $E(m, l)$ :

$$
\begin{aligned}
e(m l) & \equiv \frac{E(m, l)+\frac{m}{4}}{E(m=0, l)}=1-\frac{6 m l}{\pi}-\frac{6(m l)^{2}}{\pi^{2}} \ln (\tilde{\chi} m l) \\
& -\frac{12}{\pi} \sum_{k=1}^{\infty}\left(\sqrt{(m l)^{2}+(\pi k)^{2}}-\pi k-\frac{(m l)^{2}}{2 \pi k}\right) .
\end{aligned}
$$

Numerically very close agreement of these ratios up to $\mathcal{O}\left(10^{-5}\right)-\mathcal{O}\left(10^{-6}\right)$ is found. Their difference increases for large $m l$ as $(m l)^{2}$; this arises from our imperfect knowledge of the number $\ln \tilde{\chi}$.

\subsection{Renormalized vacuum stress tensor}

\subsection{1 $\left\langle T^{11}\right\rangle$}

From eq. 2.91) we find for the internal region

$$
\left\langle T^{11}\right\rangle_{\text {reg }}(m, l, n, a)=\frac{2}{a^{2}} \sum_{k=1}^{N-1} \frac{\sin ^{2}\left(\frac{\pi k}{2 N}\right)}{\sqrt{(m l)^{2}+4 N^{2} \sin ^{2}\left(\frac{\pi k}{2 N}\right)}}+A(m, l, n, a)
$$


where the lattice artefact term

$$
A(m, l, n, a) \equiv-\frac{2}{a^{2}} \sum_{k=1}^{N-1} \frac{\sin ^{4}\left(\frac{\pi k}{2 N}\right) \cos \left(\frac{2 \pi k n}{N}\right)}{\sqrt{(m l)^{2}+4 N^{2} \sin ^{2}\left(\frac{\pi k}{2 N}\right)}}
$$

has already been mentioned. This term has no counterpart in the continuum theory and it is unphysical. As discussed in Appendix B it makes no contribution to the Casimir force. The artefact $A$ diverges as $a \rightarrow 0$ and must be subtracted - but not yet for there is some interplay (or more precisely a cancellation) between the leading divergence in $A$ and a nonleading divergence in the first sum in eq. (3.31).

For zero mass $m=0$ one can express eq. (3.31) using eqs. (3.3), (B.18) in the form

$$
\left\langle T^{11}\right\rangle_{r e g}(l, n, a)=\frac{2}{\pi a^{2}}-\frac{\pi}{24 l^{2}}+f(l, n, a)+\mathcal{O}\left(a^{2}\right),
$$

where the lattice function $f(l, n, a)$ is defined by eq. (B.19). Because $f \rightarrow 0$ as $a \rightarrow 0$ in the internal region $0<x<l$ the renormalized continuum tensor for $m=0$ in this region is

$$
\left\langle T^{11}\right\rangle=-\frac{\pi}{24 l^{2}}=-\frac{\partial E(l)}{\partial l}
$$

as it should be (recall eq. (3.9)). For any $m \geq 0$ the continuum $\left\langle T^{11}\right\rangle$ is constant between parallel Dirichlet planes in arbitrary dimension (see e.g. ref. [10]). This constant value of $\left\langle T^{11}\right\rangle$ equals the Casimir force/area on the enclosing Dirichlet boundaries when no force acts from outside on these boundaries (because e.g. the external region is infinite).

At the lattice level note that the physical first term in eq. (3.31) is indeed independent of lattice position $n$. Thus the lattice formulation has already scored a success in reproducing this known continuum feature:

$$
\left\langle T^{11}\right\rangle_{\text {phys }}=\left\langle T^{11}\right\rangle(m, l, a)=\frac{2}{a^{2}} \sum_{k=1}^{N-1} \frac{\sin ^{2}\left(\frac{\pi k}{2 N}\right)}{\sqrt{(m l)^{2}+4 N^{2} \sin ^{2}\left(\frac{\pi k}{2 N}\right)}} .
$$

On the other hand the lattice artefact second term in eq. (3.31) does depend on $n$ - another indication of its unphysical nature.

It is not difficult to see that $\left\langle T^{11}\right\rangle(m, l, a)$ coincides with the nonrenormalized Casimir force obtained from the nonrenormalized global vacuum energy. Thus on the lattice one obtains the same Casimir force using global and local methods, just as one does in the continuum formulation. Because we know the $a \rightarrow 0$ divergent structure of eq. (3.35) from the global problem we can write down immediately the renormalized result

$$
\begin{aligned}
\left\langle T^{11}\right\rangle(m, l, \mu) & =\lim _{a \rightarrow 0}\left(\frac{2}{a^{2}} \sum_{k=1}^{N-1} \frac{\sin ^{2}\left(\frac{\pi k}{2 N}\right)}{\sqrt{(m l)^{2}+4 N^{2} \sin ^{2}\left(\frac{\pi k}{2 N}\right)}}\right. \\
& \left.-\frac{2}{\pi a^{2}}+\frac{1}{2 a l}-\frac{m^{2}}{4 \pi} \ln (\mu a)\right) .
\end{aligned}
$$


Of course the arbitrary parameter $\mu$ is again present.

Physically fixing $\mu$

The condition chosen here to fix $\mu$ is

$$
\left\langle T^{11}\right\rangle(m, l)=-\frac{\partial E(m, l)}{\partial l}
$$

where $E(m, l)$ is the fixed- $\mu$ vacuum energy (3.25). Equivalently

$$
\lim _{l \rightarrow \infty}\left\langle T^{11}\right\rangle(m, l)=0
$$

It is important to emphasize that in general

$$
\left\langle T^{11}\right\rangle(m, l, \mu) \neq-\frac{\partial E(m, l, \mu)}{\partial l},
$$

assuming one uses the same $\mu$ on both sides. Directly from eq. (3.26) we obtain the fixed- $\mu$ vacuum tensor

$$
\begin{aligned}
&\left\langle T^{11}\right\rangle(m, l)=-\frac{\partial E(m, l)}{\partial l} \\
&=\lim _{a \rightarrow 0}\left(\frac{2}{a^{2}} \sum_{k=1}^{N-1} \frac{\sin ^{2}\left(\frac{\pi k}{2 N}\right)}{\sqrt{(m l)^{2}+4 N^{2} \sin ^{2}\left(\frac{\pi k}{2 N}\right)}}-\frac{2}{\pi a^{2}}+\frac{1}{2 a l}-\frac{m^{2}}{4 \pi} \ln (\tau m a)\right)
\end{aligned}
$$

where

$$
\ln (\tau)=\ln (\chi)+1=-1.579438 \ldots .
$$

Here fixing $\mu$ has led to $\mu=\tau m$. In the global vacuum energy it was $\mu=\chi m$.

With the $V(n) \neq 0$ system of sec. 4 in mind let us say something more about the renormalization of eq. (3.31). Subtract the lattice artefact term $A$ (using eq. (B.26) for it) in addition to the other subtractions in eq. (3.36):

$$
\begin{array}{r}
\left\langle T^{11}\right\rangle(m, l, \mu)=\lim _{a \rightarrow 0}\left(\left\langle T^{11}\right\rangle_{r e g}(m, l, n, a)-\frac{2}{\pi a^{2}}-\frac{m^{2}}{4 \pi} \ln (\mu a)\right. \\
\left.-f(l, n, a)-\frac{m^{2}}{8} h\left(\frac{l}{a}, n\right)\right)
\end{array}
$$

or with $\mu=\tau m$

$$
\begin{array}{r}
\left\langle T^{11}\right\rangle(m, l)=\lim _{a \rightarrow 0}\left(\left\langle T^{11}\right\rangle_{r e g}(m, l, n, a)-\frac{2}{\pi a^{2}}-\frac{m^{2}}{4 \pi} \ln (\tau m a)\right. \\
\left.-f(l, n, a)-\frac{m^{2}}{8} h\left(\frac{l}{a}, n\right)\right),
\end{array}
$$


where $f, h$ are defined in Appendix B. As indicated both of these functions depend on position $x=n a$. However, in the continuum limit they both vanish everywhere except on the boundaries $n=0, N$ where they take values independent of $l: f \rightarrow$ const $/ a^{2}$ while $h \rightarrow 2 / \pi$. Subtracting this position dependence in eq. (3.43) yields a $\left\langle T^{11}\right\rangle$ independent of lattice position. To show this we rewrite eq. (3.43) in the form

$$
\begin{aligned}
\left\langle T^{11}\right\rangle(m, l)= & \lim _{a \rightarrow 0} \sum_{k=1}^{N-1}\left\{\frac{2 N^{2} \sin ^{2}\left(\frac{\pi k}{2 N}\right)}{l^{2} \sqrt{(m l)^{2}+4 N^{2} \sin ^{2}\left(\frac{\pi k}{2 N}\right)}}-\frac{N}{l^{2}} \sin \left(\frac{\pi k}{2 N}\right)+\frac{m^{2}}{8 N \sin \left(\frac{\pi k}{2 N}\right)}\right. \\
& -\frac{2 N^{2} \sin ^{4}\left(\frac{\pi k}{2 N}\right) \cos \left(\frac{2 \pi k x}{l}\right)}{l^{2} \sqrt{(m l)^{2}+4 N^{2} \sin ^{2}\left(\frac{\pi k}{2 N}\right)}}+\frac{N}{l^{2}} \sin ^{3}\left(\frac{\pi k}{2 N}\right) \cos \left(\frac{2 \pi k x}{l}\right) \\
& \left.-\frac{m^{2}}{8 N} \sin \left(\frac{\pi k}{2 N}\right) \cos \left(\frac{2 \pi k x}{l}\right)\right\}-\frac{\pi}{24 l^{2}}-\frac{m^{2}}{4 \pi} \ln (\tilde{\tau} m l) \\
= & -\frac{\pi}{24 l^{2}}-\frac{m^{2}}{4 \pi} \ln (\tilde{\tau} m l)+\frac{1}{2 l^{2}} \sum_{k=1}^{\infty}\left(\frac{(\pi k)^{2}}{\sqrt{(m l)^{2}+(\pi k)^{2}}}-\pi k+\frac{(m l)^{2}}{2 \pi k}\right),
\end{aligned}
$$

with

$$
\begin{aligned}
\ln (\tilde{\tau}) & =\ln (\tau)+\frac{\pi}{2} 0.52125 \ldots \\
& =\ln (\tilde{\chi})+1=-0.76066 \ldots .
\end{aligned}
$$

The two terms following the first term in curly brackets are the first two terms of its expansion in $m^{2}$ multiplied by (-1). This removes all $a \rightarrow 0$ divergences and the resulting limiting value (second equality) follows straightforwardly. Expansion of the latter in powers of $m l$ yields

$$
\begin{aligned}
\left\langle T^{11}\right\rangle(m, l) & =-\frac{m^{2}}{4 \pi} \ln (\tilde{\tau} m l)+\frac{\pi}{2 l^{2}} \sum_{\substack{\nu=0 \\
\nu \neq 1}}^{\infty} d_{\nu}\left(\frac{m l}{\pi}\right)^{2 \nu} \zeta(2 \nu-1) \quad, \text { with } \\
d_{\nu} & =\left(\begin{array}{c}
-1 / 2 \\
\nu
\end{array}\right)=(-1)^{\nu} \frac{\Gamma(1 / 2+\nu)}{\nu ! \sqrt{\pi}} .
\end{aligned}
$$

In fig. 2 we plot the ratio $\left\langle T^{11}\right\rangle(m, l) /\left\langle T^{11}\right\rangle(m=0, l)$ versus $m l$. The exponential fall-off of this function with increasing $m l$ is evident.

\subsection{2 $\left\langle T^{00}\right\rangle$}

From eq. (2.90) we have

$$
\begin{aligned}
\left\langle T^{00}\right\rangle_{\text {reg }}(m, l, n, a)= & \frac{E_{\text {reg }}(m, l, a)}{l}-\frac{m^{2}}{2} \sum_{k=1}^{N-1} \frac{\cos \left(\frac{2 \pi k n}{N}\right)}{\sqrt{(m l)^{2}+4 N^{2} \sin ^{2}\left(\frac{\pi k}{2 N}\right)}} \\
& +A(m, l, n, a) .
\end{aligned}
$$




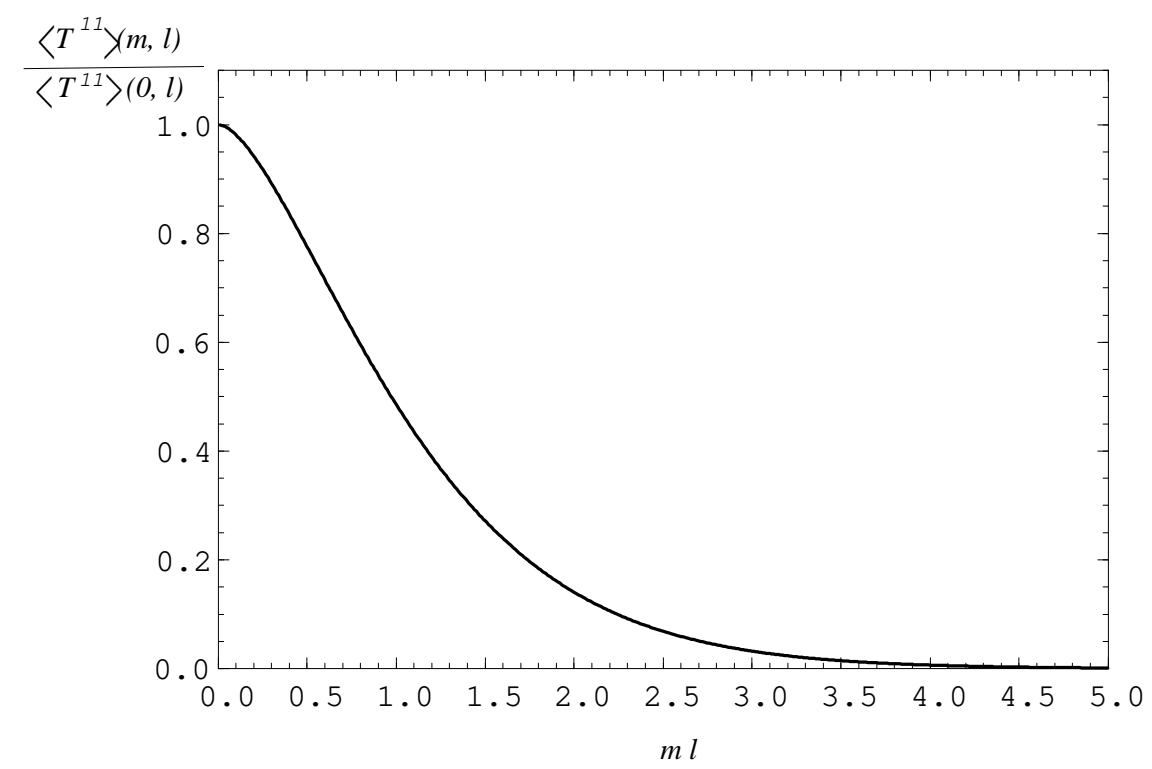

Figure 2: The ratio $\left\langle T^{11}\right\rangle(m, l) /\left\langle T^{11}\right\rangle(0, l)$ with $\ln \tilde{\tau}=-0.76066$ versus $m l$. Eq. (3.44) is summed to $k=1000$.

The property

$$
\sum_{n=1}^{N} \cos \left(\frac{2 \pi k n}{N}\right)=0 \quad \text { for } \quad k \neq 0
$$

then yields immediately (compare eq. (2.83))

$$
a \sum_{n=1}^{N}\left\langle T^{00}\right\rangle_{\text {reg }}(m, l, n, a)=E_{\text {reg }}(m, l, a) .
$$

Let us now renormalize eq. (3.47).

The second term in eq. (3.47) remains finite in the limit $N \rightarrow \infty$ away from the boundaries

$$
\lim _{N \rightarrow \infty}\left(\sum_{k=1}^{N-1} \frac{\cos \left(\frac{2 \pi k n}{N}\right)}{\sqrt{(m l)^{2}+4 N^{2} \sin ^{2}\left(\frac{\pi k}{2 N}\right)}}\right)=\sum_{k=1}^{\infty} \frac{\cos \left(\frac{2 \pi k x}{l}\right)}{\sqrt{(m l)^{2}+(\pi k)^{2}}} .
$$

This series converges for $0<x<l$. However its behavior near the boundaries is $\ln x$ and $\ln (l-x)$ for $x \rightarrow 0$ and $x \rightarrow l$ respectively (where the series diverges logarithmically). The renormalized energy density

$$
\begin{aligned}
& T^{00}(m, l, x, \mu)=\frac{E(m, l, \mu)}{l}-\frac{m^{2}}{2} \sum_{k=1}^{\infty} \frac{\cos \left(\frac{2 \pi k x}{l}\right)}{\sqrt{(m l)^{2}+(\pi k)^{2}}} \\
& \quad=\lim _{a \rightarrow 0}\left(\left\langle T^{00}\right\rangle_{\text {reg }}\left(m, l, \frac{x}{a}, a\right)-\frac{2}{\pi a^{2}}+\frac{m^{2}}{4 \pi} \ln (\mu a)-f\left(l, \frac{x}{a}, a\right)-\frac{m^{2}}{8} h\left(\frac{l}{a}, \frac{x}{a}\right)\right),
\end{aligned}
$$




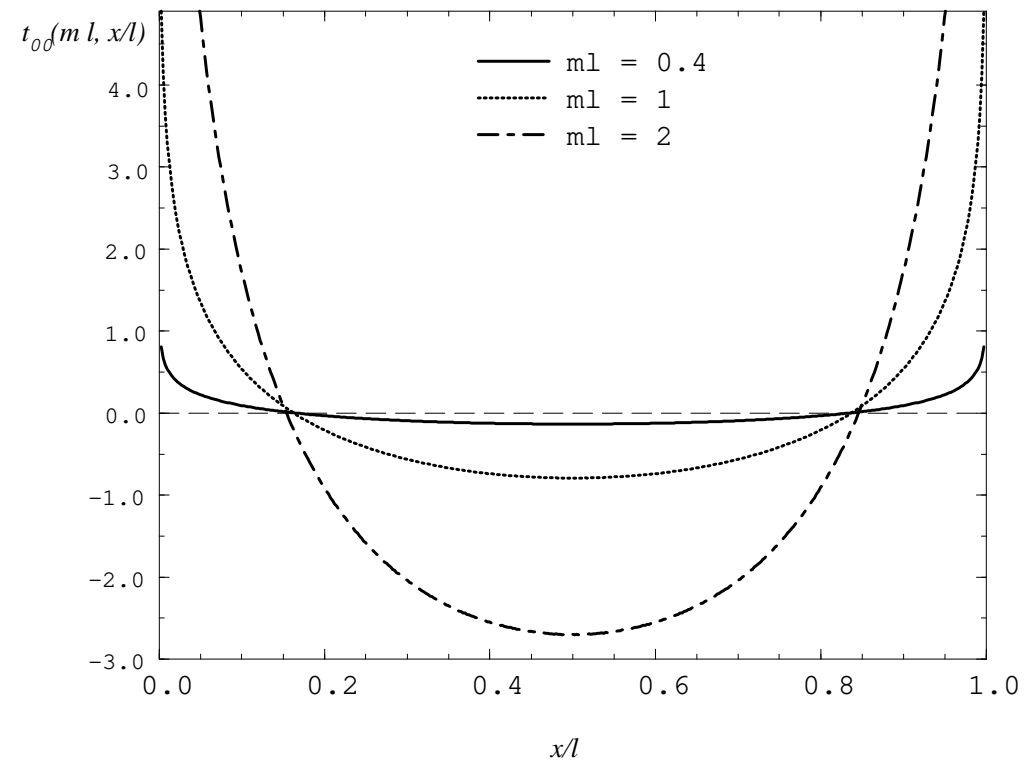

Figure 3: The function eq. (3.54) summed to $k=1000$ over the interval $0<x / l<1$.

therefore contains the expected boundary divergences. Fixing $\mu$ by $\mu=\chi m$ we obtain

$$
\begin{aligned}
& T^{00}(m, l, x)=\frac{E(m, l)}{l}-\frac{m^{2}}{2} \sum_{k=1}^{\infty} \frac{\cos \left(\frac{2 \pi k x}{l}\right)}{\sqrt{(m l)^{2}+(\pi k)^{2}}} \\
& \quad=\lim _{a \rightarrow 0}\left(\left\langle T^{00}\right\rangle_{\text {reg }}\left(m, l, \frac{x}{a}, a\right)-\frac{2}{\pi a^{2}}+\frac{m^{2}}{4 \pi} \ln (\chi m a)-f\left(l, \frac{x}{a}, a\right)-\frac{m^{2}}{8} h\left(\frac{l}{a}, \frac{x}{a}\right)\right) .
\end{aligned}
$$

Summing eq. (3.52) over the lattice $0<x<l$ we find with the help of eqs. (B.11), (B.25)

$$
\begin{aligned}
\sum_{n=1}^{N} a & \left(\left\langle T^{00}\right\rangle_{\text {reg }}(m, l, n, a)-\frac{2}{\pi a^{2}}+\frac{m^{2}}{4 \pi} \ln (\chi m a)-f(l, n, a)-\frac{m^{2}}{8} h\left(\frac{l}{a}, n\right)\right) \\
& =E_{\text {reg }}(m, l, a)-\frac{2 l}{\pi a^{2}}+\frac{1}{2 a}+\frac{m^{2} l}{4 \pi} \ln (\chi m a),
\end{aligned}
$$

which is the renormalized global vacuum energy (3.23) as it should be. This entitles us to assign parameter $\chi$ the same value it has in eq. (3.23). Here we do not have a condition to impose which fixes $\chi$ nor do we need one. The connection (3.53) is quite sufficient.

The energy density $\left\langle T^{00}\right\rangle$ is in contrast with the constant $\left\langle T^{11}\right\rangle$ strongly position dependent, especially important being logarithmic divergences at the boundaries. To display this we plot in fig. 3 the quantity

$$
t^{00}\left(m l, \frac{x}{l}\right) \equiv \frac{\left\langle T^{00}\right\rangle(m, l, x)}{\left\langle T^{00}\right\rangle(m=0, l)}-\frac{E(m, l)}{E(m=0, l)}
$$




$$
=\frac{12(m l)^{2}}{\pi} \sum_{k=1}^{\infty} \frac{\cos \left(\frac{2 \pi k x}{l}\right)}{\sqrt{(m l)^{2}+(\pi k)^{2}}}
$$

versus $x / l$ for three values of $m l$. The boundary divergences in $\left\langle T^{00}\right\rangle$ are inseparable from the boundaries themselves [12]. It is gratifying that the lattice formalism very closely reproduces [2, 3] this crucially-important and prominent feature of continuum QFT.

\section{One dimensional lattice with Bessel potential background}

Continuing on the 1D lattice with Dirichlet point boundaries at $x_{1}=0, x_{2}=l$ and $x_{3}=L$ (in this section it will be convenient to make this change of notation) we now introduce a background potential $V(x) \geq 0$. The Dirichlet boundaries at $x=x_{1}, x_{2}, x_{3}$ are embedded in $V(x)$. For convenience we shall in this section combine $V(x)$ and the mass term into a single potential

$$
U(x) \equiv m^{2}+V(x) \text {. }
$$

In subsection 4.1 we compute the lattice vacuum stress tensor and vacuum energy for the region $x_{1} \leq x \leq x_{2}$ between adjacent Dirichlet boundaries embedded in an arbitrary background potential $V(x)$ which vanishes as $x \rightarrow \infty$. Then in subsection 4.2 we choose $V(x)$ to be the potential

$$
V(x)=\frac{\alpha^{2}-\frac{1}{4}}{\left(x-x_{0}\right)^{2}}, \alpha \geq 1 / 2
$$

and begin the principal numerical work of this paper. We compute numerically $\left\langle T^{\mu \nu}\right\rangle$ in $x_{1}<x<x_{3}$ and the global vacuum energy in this same region. Then we compute the Casimir force on the middle Dirichlet boundary at $x=x_{2}=l$ first as the jump in $\left\langle T^{11}\right\rangle$ at this point, and second using the global method. Very close agreement between these two forces (which should be the same, of course) is found. This is a crucial test of our lattice method, and it succeeds. A variety of other checks and tests will also be displayed in subsection 4.2.

We remind the reader that the potential (4.2) has a specific physical interpretation. It defines what the authors call a "Bessel boundary" [2, 5] - i.e. semitransparent surface texture represented by $V(x)$ attached to a core Dirichlet boundary at $x=x_{0}$. This entire boundary was absent in the analysis of sec. 3. Parameter $\alpha \geq 1 / 2$ controls the amount of semitransparent material present and the potential $V(x)$ extends from $x_{0}$ into $x>x_{0}$ as far as we choose to let it. [We can also let $V(x)$ extend arbitrarily far into $x<x_{0}$ - and in fact should do so if we wanted to calculate the Casimir force on the Bessel wall - but so far as this section is concerned, we 
need not bother about the region $x<x_{0}$.] Without the quantum field the classical background material represented by $V(x)$ would not interact directly with the classical boundaries at $x=x_{1}, x_{2}, x_{3}$ nor would these objects interact with one another. However, when the quantum field $\phi$ is present it is distorted by $V(x)$ and by each of the hard boundaries at $x=x_{1}, x_{2}, x_{3}$. Back forces are then exerted by $\phi$ on the extended structure $V(x)$ and on each of the hard boundaries. Forces involving all parts of the spatial background (including the material represented by $V(x)$ ) come into existence. Thus we have a highly nontrivial Casimir system to study.

\subsection{Renormalized vacuum stress tensor for semihard $V(x)$}

\subsection{1 $\left\langle T^{11}\right\rangle$}

For the region $x_{1} \leq x \leq x_{2}$ we have from eqs. (2.77), the lattice formula for arbitrary background potential $V(n)$

$$
\begin{aligned}
\left\langle T^{11}\right\rangle_{\text {reg }}\left(x_{1}, x_{2}, x_{1}+n a, a\right)= & \sum_{k=1}^{N-1}\left(\frac{\left(\varepsilon_{k}^{2}-U\left(x_{1}+n a\right)\right)\left(v_{n}^{k}\right)^{2}}{2 a \varepsilon_{k}}\right. \\
& \left.+\frac{\left(v_{n+1}^{k}\right)^{2}-2\left(v_{n}^{k}\right)^{2}+\left(v_{n-1}^{k}\right)^{2}}{8 a^{3} \varepsilon_{k}}\right) .
\end{aligned}
$$

Here $x_{2}-x_{1}=N a \equiv l$. As we show in Appendix $\mathrm{C}$ this expression can be renormalized for arbitrary $V(n)$. The renormalized lattice $\left\langle T^{11}\right\rangle$ is (prior to letting $a \rightarrow 0$ )

$$
\begin{aligned}
\left\langle T^{11}\right\rangle\left(x_{1}, x_{2}, x_{1}+n a, a, \mu\right)= & \left\langle T^{11}\right\rangle_{\text {reg }}\left(x_{1}, x_{2}, x_{1}+n a, a\right)-\frac{2}{\pi a^{2}}-f(l, n, a) \\
& -\frac{U\left(x_{1}+n a\right)}{4 \pi} \ln (\mu a)-\frac{U\left(x_{1}+n a\right)}{8} h\left(\frac{l}{a}, n\right)(4.4)
\end{aligned}
$$

where $h$ and $f$ are defined by eqs. (B.12), (B.21) respectively. As in the corresponding lattice formula (3.36) for $V(n)=0$ the renormalization process introduces dependence on an arbitrary renormalization mass parameter $\mu$ (as always because of the need to subtract a lattice sum diverging logarithmically as the lattice constant $a \rightarrow 0$ ). Such an arbitrary renormalization parameter introduces ambiguity, of course. Fortunately, because of the way $\left\langle T^{11}\right\rangle$ depends on $\mu$ in eq. (4.4) this parameter does not contribute to Casimir forces on e.g. the boundaries at $x=x_{1}$ and $x_{2}$. The latter are determined by the jump in $\left\langle T^{11}\right\rangle$ across these points. If the background potential $V(x)$ is continuous across a boundary, then the term $U(x) \ln \mu a$ takes on the same value on both sides of this boundary: there is no jump in $\left\langle T^{11}\right\rangle$ and therefore no Casimir force. Only if $V(x)$ makes a jump at a boundary will the Casimir force on this boundary depend on $\mu$. Because Casimir forces are the principal experimental signature of the distortion of the quantum field by its interaction with all background structure the importance of $F_{C a s}$ not depending on $\mu$ 
for continuous background potential $V(x)$ is clear. This observation also highlights the importance of not merely considering the internal region in studying a Casimir problem. To have a well-posed problem external regions have to be considered as well.

Quite another question to be answered is this: Is the renormalized vacuum stress tensor itself well-defined? $\left\langle T^{\mu \nu}\right\rangle$ is after all our basic local mathematical instrument for representing/investigating the physical properties of the system. If $\mu$ in eq. (4.4) is truly arbitrary then $\left\langle T^{\mu \nu}\right\rangle$ is not unique. What are the consequences of this? Is the nonuniqueness associated with $\mu$ relatively trivial or is it symptomatic of something deeper? Even if $\mu$ does not influence Casimir forces it does influence the energy density $\left\langle T^{00}\right\rangle$ and the total vacuum energy.

We see no compelling reason to view $\mu$ as being, in some sense, a universal constant. If it were, then why would it be absent for $U(x)=0$ ? Thus in sec. 3 where $V(x)=0$ we have fixed this ambiguity by an ad hoc but reasonable condition

$$
\lim _{l \rightarrow \infty}\left\langle T^{11}\right\rangle(m, l)=0
$$

Physically this means that there is no contribution to the Casimir force on the hard boundary at $x=l$ coming from the infinitely extended region $x>l$.

Here with $x$-dependent potential $V(x)>0$ we choose what we believe is the natural extension of the scheme in sec. 3. We set

$$
\mu^{2}=\tau^{2} U(x)=\tau^{2}\left[m^{2}+V(x)\right]
$$

which of course reduces to the ansatz $\mu=\tau m$ for $V(x)=0$. Then eq. (4.4) becomes

$$
\begin{gathered}
\left\langle T^{11}\right\rangle\left(x_{1}, x_{2}, x_{1}+n a, a\right)=\left\langle T^{11}\right\rangle_{r e g}\left(x_{1}, x_{2}, x_{1}+n a, a\right)-\frac{2}{\pi a^{2}}-f(l, n, a) \\
-\frac{U\left(x_{1}+n a\right)}{8 \pi} \ln \left(\tau^{2} U\left(x_{1}+n a\right) a^{2}\right)-\frac{U\left(x_{1}+n a\right)}{8} h\left(\frac{l}{a}, n\right) .
\end{gathered}
$$

Note that $\tau$ here still does not influence any hard-boundary Casimir force as long as $V(x)$ is continuous. Because $\tau$ is a constant we can fix it by going to spatial infinity where (we assume) $V(x) \rightarrow 0$ so that $U(x) \rightarrow m^{2}$ :

$$
\lim _{x \rightarrow \infty} \lim _{x_{2} \rightarrow \infty}\left\langle T^{11}\right\rangle\left(x_{1}, x_{2}, x, \mu\right)=0 .
$$

Here $x_{1}<x<x_{2}$ prior to moving $x_{2}=l$ out to infinity and then following with $x$. This condition is essentially the same as that posed in the $V(x)=0$ case in sec. 3 and, just as in eq. (3.41), we obtain the fixed $\tau$

$$
\ln \tau=-0.76066 \ldots-\frac{\pi}{2} 0.52125 \ldots=-1.579438 \ldots
$$

Eq. (4.7) generalizes eq. (3.43) in the sense $m^{2} \rightarrow U(x)$. Now in the continuum limit with $x=x_{1}+n a$ held constant, $l=x_{2}-x_{1}$ and $\hat{x}=x-x_{1}$

$$
\left\langle T^{11}\right\rangle\left(x_{1}, x_{2}, x\right)=\lim _{a \rightarrow 0}\left(\left\langle T^{11}\right\rangle_{r e g}\left(x_{1}, x_{2}, x, a\right)-\frac{2}{\pi a^{2}}-f\left(l, \frac{\hat{x}}{a}, a\right)\right.
$$




$$
\left.-\frac{U(x)}{8 \pi} \ln \left(\tau^{2} U(x) a^{2}\right)-\frac{U(x)}{8} h\left(\frac{l}{a}, \frac{\hat{x}}{a}\right)\right) .
$$

\subsection{2 $\left\langle T^{00}\right\rangle$}

The lattice formula for $\left\langle T^{00}\right\rangle$ is

$$
\left\langle T^{00}\right\rangle_{\text {reg }}\left(x_{1}, x_{2}, x_{1}+n a, a\right)=\sum_{k=1}^{N-1}\left(\frac{\varepsilon_{k}}{2 a}\left(v_{n}^{k}\right)^{2}+\frac{\left(v_{n+1}^{k}\right)^{2}-2\left(v_{n}^{k}\right)^{2}+\left(v_{n-1}^{k}\right)^{2}}{8 a^{3} \varepsilon_{k}}\right) .
$$

The renormalized lattice vacuum energy generalizing eq. (3.53) is

$$
\begin{aligned}
& \left\langle T^{00}\right\rangle\left(x_{1}, x_{2}, x_{1}+n a, a, \mu\right)=\left\langle T^{00}\right\rangle_{r e g}\left(x_{1}, x_{2}, x_{1}+n a, a\right)-\frac{2}{\pi a^{2}}-f(l, n, a) \\
& +\frac{U\left(x_{1}+n a\right)}{8 \pi} \ln \left(\mu^{2} a^{2}\right)-\frac{U\left(x_{1}+n a\right)}{8} h\left(\frac{l}{a}, n\right) .
\end{aligned}
$$

With $\mu^{2}=\chi^{2} U(x)$

$$
\begin{gathered}
\left\langle T^{00}\right\rangle\left(x_{1}, x_{2}, x_{1}+n a, a\right)=\left\langle T^{00}\right\rangle_{r e g}\left(x_{1}, x_{2}, x_{1}+n a, a\right)-\frac{2}{\pi a^{2}}-f(l, n, a) \\
+\frac{U\left(x_{1}+n a\right)}{8 \pi} \ln \left(\chi^{2} U\left(x_{1}+n a\right) a^{2}\right)-\frac{U\left(x_{1}+n a\right)}{8} h\left(\frac{l}{a}, n\right),
\end{gathered}
$$

where

$$
\ln \chi=\ln \tau-1=-2.579438 \ldots
$$

In the continuum limit

$$
\begin{array}{r}
\left\langle T^{00}\right\rangle\left(x_{1}, x_{2}, x\right)=\lim _{a \rightarrow 0}\left(\left\langle T^{00}\right\rangle_{r e g}\left(x_{1}, x_{2}, x, a\right)-\frac{2}{\pi a^{2}}-f\left(l, \frac{\hat{x}}{a}, a\right)\right. \\
\left.+\frac{U(x)}{8 \pi} \ln \left(\chi^{2} U(x) a^{2}\right)-\frac{U(x)}{8} h\left(\frac{l}{a}, \frac{\hat{x}}{a}\right)\right) .
\end{array}
$$

\subsubsection{Renormalized vacuum energy}

The lattice vacuum energy in the interval $x_{1}<x<x_{2}$

$$
E_{r e g}\left(x_{1}, x_{2}, a\right)=\frac{1}{2} \sum_{k=1}^{N-1} \varepsilon_{k}
$$

is renormalized in Appendix $\mathrm{C}$ with the result

$$
E\left(x_{1}, x_{2}, a, \mu\right)=E_{r e g}\left(x_{1}, x_{2}, a\right)-\frac{2\left(x_{2}-x_{1}\right)}{\pi a^{2}}+\frac{1}{2 a}+\frac{1}{4 \pi} \int_{x_{1}}^{x_{2}} U(x) d x \ln (\mu a) .
$$


Assuming $E=\int\left\langle T^{00}\right\rangle d x$ we find from eq. (4.13) the same renormalized lattice vacuum energy

$$
\begin{aligned}
E\left(x_{1}, x_{2}, a\right) & =E_{r e g}\left(x_{1}, x_{2}, a\right)-\frac{2\left(x_{2}-x_{1}\right)}{\pi a^{2}}+\frac{1}{2 a} \\
& +\frac{1}{8 \pi} \int_{x_{1}}^{x_{2}} U(x) \ln \left(\chi^{2} U(x) a^{2}\right) d x
\end{aligned}
$$

Note that in eqs. (4.17), (4.18) one can use in place of the integral the lattice sum

$$
\frac{1}{16 \pi}\left(\sum_{n=0}^{N-1}+\sum_{n=1}^{N}\right) a U\left(x_{1}+n a\right) \ln \left(\chi^{2} U\left(x_{1}+n a\right) a^{2}\right)
$$

The difference vanishes when $a \rightarrow 0$. In the continuum limit we define

$$
E\left(x_{1}, x_{2}\right):=\lim _{a \rightarrow 0} E\left(x_{1}, x_{2}, a\right) .
$$

\subsection{Numerical evaluation using the Bessel potential}

Now we proceed to a numerical investigation of the renormalized $\left\langle T^{\mu \nu}\right\rangle$ and $E\left(x_{1}, x_{2}\right)$ defined above for the background Bessel potential (4.2). The core of the Bessel boundary is at $x=x_{0}$ and the surface texture represented by $V(x)$ extends to the right from $x=x_{0}$. We generally choose $x_{0}=-0.01$ and position the first Dirichlet boundary at $x_{1}=0$. The other two Dirichlet boundaries are further to the right at $x=x_{2}, x_{3}>0$. Before presenting our numerical results it is perhaps worth saying again how they are obtained. First the lattice mode equation (2.46) has to be solved numerically for the lattice modes $v_{n}^{k}$ and their energy eigenvalues $\varepsilon_{k}^{2}$. Vanishing boundary conditions at each of the hard boundaries are additionally imposed on the $v_{n}^{k}$. A given calculation is performed for specific values of lattice constant $a$, of parameter $\alpha$ in eq. (4.2), of mass $m$ and of positions $x_{1}, x_{2}, x_{3}$. When any of these six values is changed the numerical calculation has to be redone. Given the numerical ingredients $\left\{v_{n}^{k}\right\}$ and $\left\{\varepsilon_{k}^{2}\right\}$ one can insert these ingredients into eqs. (4.4), (4.13) to obtain the lattice $\left\langle T^{\mu \nu}\right\rangle$ numerically.

\subsubsection{Renormalized $\left\langle T^{00}\right\rangle$}

Fig. 4 shows the function $\left\langle T^{00}\right\rangle$ obtained from eq. (4.13) in $x_{1}<x<x_{2}$ with $x_{0}=-0.01, x_{1}=0, x_{2}=2$ for $m=0$ and three different values of the parameter $\alpha$ in eq. (4.2): these are $\alpha=1 / 2$ (or $V(x)=0$ everywhere between the Dirichlet boundaries), $\alpha=1$ and $\alpha=5$. Note again the arbitrary length unit along the horizontal axis. For $\alpha=1 / 2$ we recover the well-known $(m=0)$-result (see e.g. ref. 10 $\left\langle T^{00}\right\rangle=\pi^{2} /\left(24 l^{2}\right)$. For $\alpha>1 / 2$ we see that $\left\langle T^{00}\right\rangle$ is (i) asymmetric about the midpoint $x=1$ because of the asymmetry of the potential (4.2) and

(ii) divergent as the boundaries are approached. To investigate this divergence 


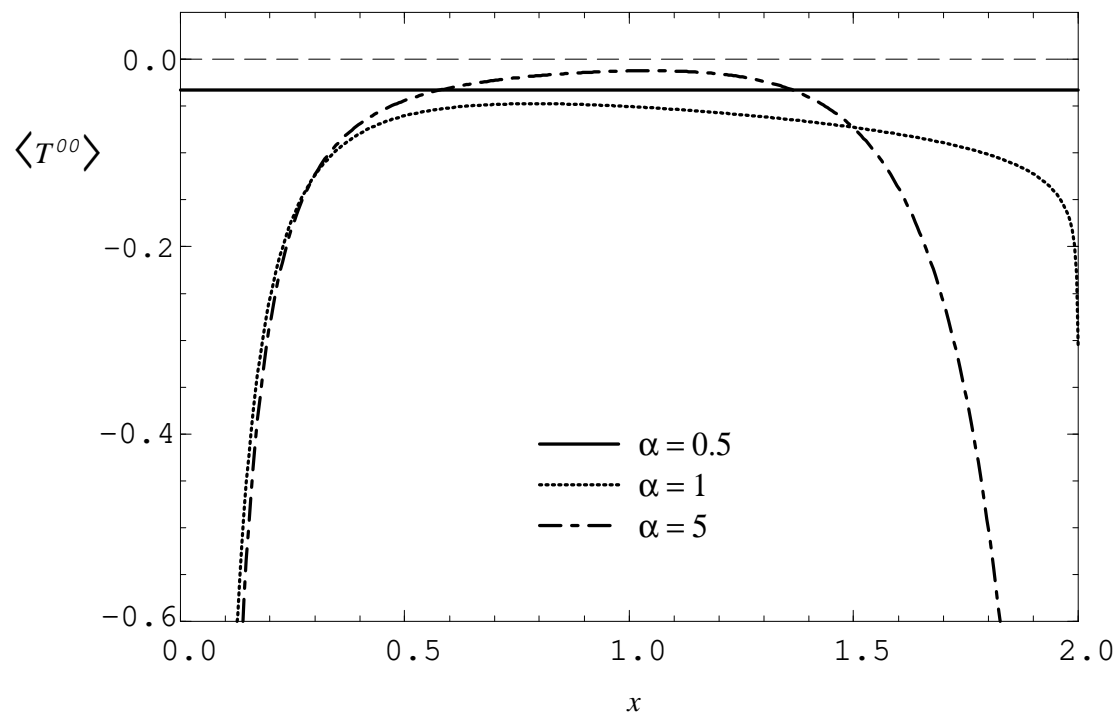

Figure 4: $\left\langle T^{00}\right\rangle$ from eq. (4.13) with $m=0, x_{0}=-0.01$ for Dirichlet boundaries at $x_{1}=0$ and $x_{2}=2$. The lattice constant is $a=1 / 400$.

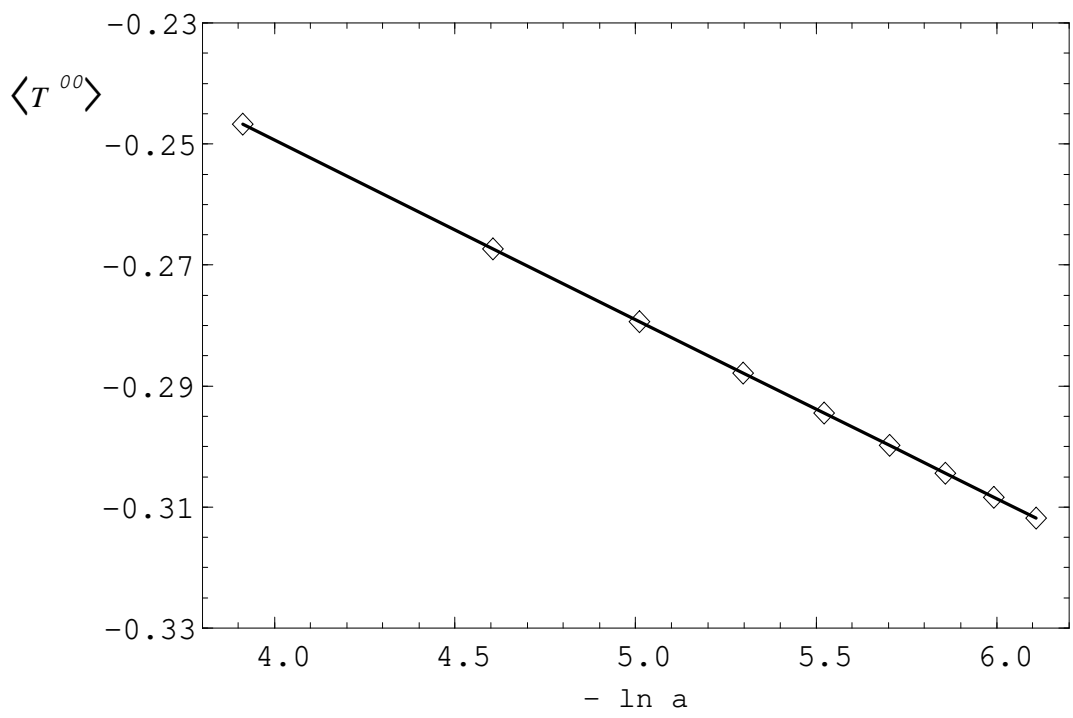

Figure 5: $\left\langle T^{00}\right\rangle$ from eq. (4.13) with $\alpha=1$ and other parameters as in fig. 4, evaluated on the right-hand boundary $x=x_{2}=2$. 


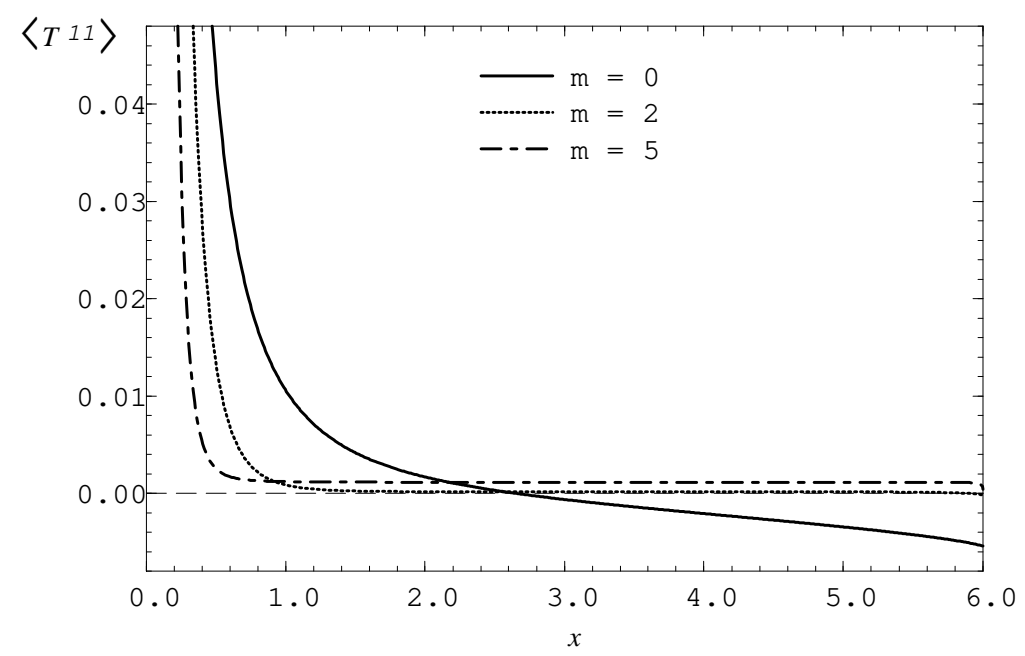

Figure 6: $\left\langle T^{11}\right\rangle$ from eq. (4.7) with $x_{0}=-0.01, \alpha=1$ for Dirichlet boundaries at $x_{1}=0$ and $x_{2}=6$. The lattice constant is $a=1 / 300$.

differently we display in fig. $5\left\langle T^{00}\right\rangle$ for $n=N$ or $x=x_{2}=2$ on the right hand boundary and $\alpha=1$ as a function of $(-\ln a)$. Clearly a logarithmic divergence is involved.

\subsubsection{Renormalized $\left\langle T^{11}\right\rangle$}

Here we use eq. (4.7) with the potential (4.2) to compute numerically the renormalized $\left\langle T^{11}\right\rangle$. First of all we wish to show what this function looks like when the Dirichlet boundaries are absent and the system consists of the scalar field coexisting with the Bessel boundary. Of course this system can only approximately be realized on a finite lattice. Again we position a Dirichlet boundary at $x=x_{1}=0$ very close to the core $x_{0}=-0.01$ of the Bessel boundary. This is done for computational reasons. We expect (and we may also conclude from our numerical results) that this hard boundary essentially disappears into the (Dirichlet like) Bessel boundary and has no significant effect on $\left\langle T^{11}\right\rangle$ away from $x>0$. Also for computational reasons we introduce a second Dirichlet boundary at $x_{2}=6$ "far" from $x=0$. We obtain the numerical results shown in fig. 6 for three different mass values $m=0,2,5$ (and $\alpha=1, a=1 / 300)$. These need to be discussed in some detail.

For "large" distance $x$ from the core of the Bessel boundary the potential (4.2) is essentially constant (and small). Thus one expects $\left\langle T^{11}\right\rangle$ for large $x$ to approach a constant value equal to $\left\langle T^{11}\right\rangle$ in free space for the same field mass $m$ (see sec. 4.2.1). This behavior is evident in fig. 6 for masses $m=2,5$. For smaller mass $m=2$ the asymptotic value for large $x$ is close to zero. For larger mass $m=5$ the asymptotic value of $\left\langle T^{11}\right\rangle$ increases noticeably. We remind the reader of the free-space vacuum 
stress tensor in one spatial dimension (see e.g. ref. [10])

$$
T_{\text {free }}^{\mu \nu}=\eta_{\mu \nu} \Lambda, \Lambda=-\frac{m^{2}}{4 \pi} \ln \frac{m}{\mu}
$$

This formula indicates that, if one may assume $m>\mu, T_{\text {free }}^{11}$ is positive and increasing with increasing $m$-just what is observed in fig. 6. But remember that we fixed $\tau$ in eq. (4.7) by requiring $\left\langle T^{11}\right\rangle$ to vanish at large distance. (The same requirement applied to $T_{\text {free }}^{\mu \nu}$ would lead to $\mu=m$ and this tensor vanishing everywhere.) This is observed in fig. 6 for $m=2$ but not for $m=5$. The mathematical reason for the $m=5$ curve not vanishing is our imperfect knowledge of the value of parameter $\tau$. The numerical consequences of this grow with $m$, one consequence being that $\left\langle T^{11}\right\rangle$ does not quite vanish for large $x$ although it should. Note also that with increasing $m$ the asymptotic value of $\left\langle T^{11}\right\rangle$ is reached in a shorter $x$ interval. This is entirely consistent with continuum results where boundary effects are known to fall off exponentially in $m x$.

The $(m=0)$-curve in fig. 6 does not approach the value $\left\langle T^{11}\right\rangle=0$ asymptotically, nor should it. For $m=0$ there is no parameter $\mu$ to fix and no asymptotic condition on $\left\langle T^{11}\right\rangle$. For $m=0$ boundary effects in $\left\langle T^{11}\right\rangle$ no longer diminish exponentially away from the $x=0$ boundary, but rather diminish as the inverse power $x^{-2}$. Consequently the boundary at $x_{3}=6$ is no longer "far away" and in fact is strongly influencing $\left\langle T^{11}\right\rangle$ in fig. 6 . To see $\left\langle T^{11}\right\rangle$ asymptotically approaching zero one must choose a substantially larger value of $x_{2}$. The $m=0$ continuum vacuum stress tensor between Dirichlet boundaries at $x=0, L$ is

$$
\left\langle T^{\mu \nu}\right\rangle=-\delta_{\mu \nu} \frac{\pi}{24 L^{2}}
$$

In fig. 6 the $(m=0)$-curve for $\left\langle T^{11}\right\rangle$ goes negative as it should.

Now let us modify the system by introducing a third boundary at $x=x_{2}=1$, leaving the other two boundaries in place at $x_{1}=0$ and $x_{3}=6$ (the previous $x_{2}$ is now renamed $\left.x_{3}\right)$. Figs. 7,8 display $\left\langle T^{11}\right\rangle$ throughout the interval $0<x<6$. One sees in fig. 7 that $F_{C a s}$ on the intermediate boundary $x_{2}=1$ defined by

$$
F_{C a s}=\left\langle T^{11}\right\rangle_{x=1-\varepsilon}-\left\langle T^{11}\right\rangle_{x=1+\varepsilon}, \varepsilon \rightarrow 0_{+}
$$

is directed to the left. This is predictable: the Bessel boundary's core $x_{0}=-0.01$ and the Dirichlet point $x_{1}=0$ are much closer to $x_{2}=1$ than is the other Dirichlet boundary $x_{3}=6$. Dirichlet objects attract in Casimir theory and the Casimir force between closer Dirichlet objects dominates. Also predictable is the decrease shown in fig. 7 of $F_{\text {Cas }}$ with increasing mass $m$. For $V(x)=0$ between Dirichlet boundaries it is known that Casimir forces weaken rapidly (exponentially) with increasing mass. There is no reason why any background potential $V(x)$ of the type considered here would qualitatively alter this general rule.

Fig. 8 shows $\left\langle T^{11}\right\rangle$ for fixed $m=0$ and three values of the strength parameter 


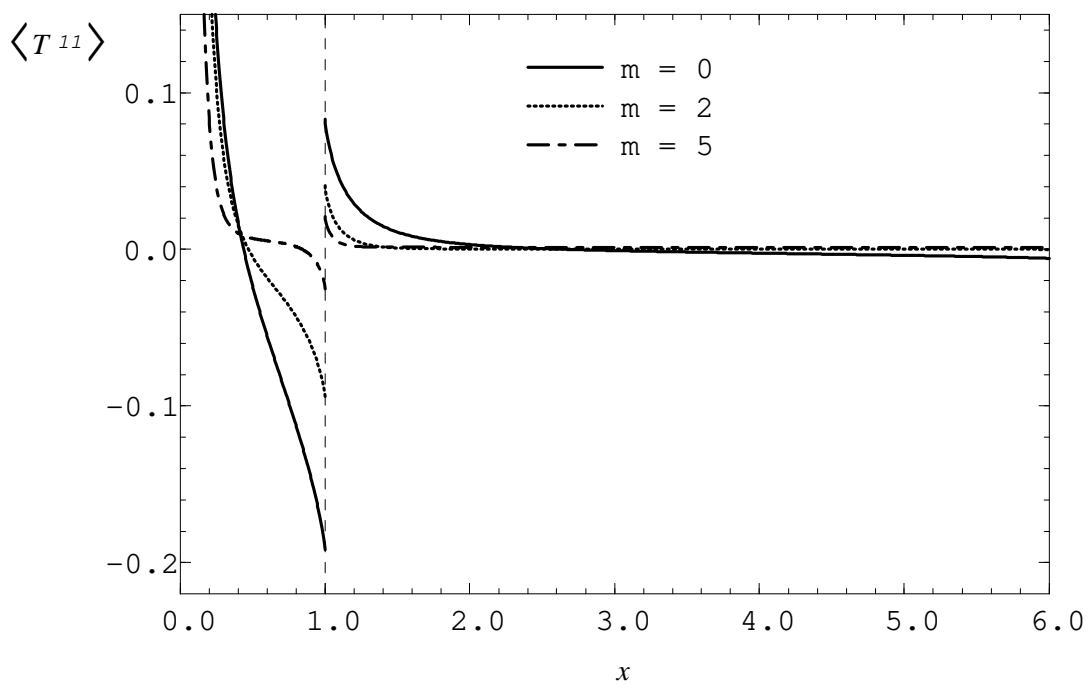

Figure 7: $\left\langle T^{11}\right\rangle$ from eq. (4.7) with $x_{0}=-0.01, \alpha=1, a=1 / 300$ for Dirichlet boundaries at $x_{1}=0, x_{2}=1$ and $x_{3}=6$.

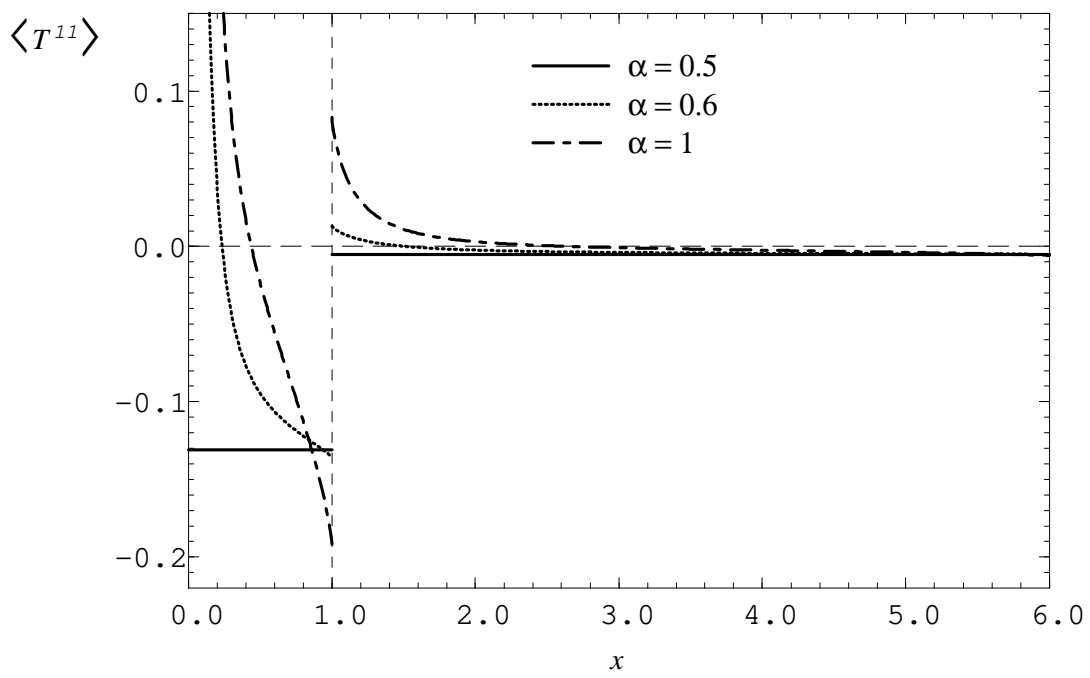

Figure 8: $\left\langle T^{11}\right\rangle$ from eq. (4.7) with $x_{0}=-0.01, m=0, a=1 / 300$ for Dirichlet boundaries at $x_{1}=0, x_{2}=1$ and $x_{3}=6$. 
$\alpha=0.5,0.6$ and 1.0 in potential $\mathrm{V}(\mathrm{x})$. Clearly $F_{\text {Cas }}$ increases with increasing $\alpha$. This too is predictable. When $\alpha=1 / 2$ and $V(x)=0$ we know that $\left\langle T^{11}\right\rangle=-\pi /\left(24 L^{2}\right)$ is constant (with $L=1$ to the left of $x_{2}=1$ and $L=5$ to the right of $x_{2}=1$ ). Increasing $\alpha$ above $\alpha=1 / 2$ causes $V(x)=0$ to jump abruptly and nonuniformly to a positive value everywhere, and obviously to a much larger average value in $0<x<1$ than in $1<x<6$. The potential $V(x)$ represents "Dirichlet material" added throughout the interval $0<x<6$. This nonuniformly added material pulls the $x_{2}=1$ boundary more strongly to the left than to the right.

\subsubsection{Renormalized vacuum energy}

These numerical calculations of the vacuum energy are based on eq. (4.18), where the integral can be done analytically for the potential (4.2). With $l=x_{2}-x_{1}$ and $b=\alpha^{2}-1 / 4$ we find

$$
\begin{aligned}
\int_{x_{1}}^{x_{2}} U(x) \ln \left(\chi^{2} U(x) a^{2}\right) d x= & \ln \left(\frac{\chi^{2} a^{2}}{l^{2}}\right) \int_{x_{1}}^{x_{2}} U(x) d x \\
& +\frac{1}{l} \int_{0}^{1} U\left(x_{1}+x l\right) l^{2} \ln \left(U\left(x_{1}+x l\right) l^{2}\right) d x
\end{aligned}
$$

and

$$
\begin{aligned}
\bar{U}\left(x_{1}, x_{2}\right) \equiv & \frac{1}{l} \int_{x_{1}}^{x_{2}}\left(m^{2}+\frac{\alpha^{2}-\frac{1}{4}}{\left(x-x_{0}\right)^{2}}\right) d x=m^{2}+\frac{\alpha^{2}-\frac{1}{4}}{\left(x_{1}-x_{0}\right)\left(x_{2}-x_{0}\right)}, \\
g\left(x_{1}, x_{2}\right) \equiv & \int_{0}^{1}\left((m l)^{2}+\frac{\alpha^{2}-\frac{1}{4}}{\left(\frac{x_{1}-x_{0}}{l}+x\right)^{2}}\right) \ln \left((m l)^{2}+\frac{\alpha^{2}-\frac{1}{4}}{\left(\frac{x_{1}-x_{0}}{l}+x\right)^{2}}\right) d x \\
= & -\frac{2 b l^{2}}{\left(x_{1}-x_{0}\right)\left(x_{2}-x_{0}\right)} \\
& +4 m l \sqrt{b}\left[\arctan \left(\frac{m\left(x_{2}-x_{0}\right)}{\sqrt{b}}\right)-\arctan \left(\frac{m\left(x_{1}-x_{0}\right)}{\sqrt{b}}\right)\right] \\
& +\frac{l\left(m^{2}\left(x_{2}-x_{0}\right)^{2}-b\right)}{x_{2}-x_{0}} \ln \left(\frac{l^{2}\left(m^{2}\left(x_{2}-x_{0}\right)^{2}+b\right)}{\left(x_{2}-x_{0}\right)^{2}}\right) \\
& -\frac{l\left(m^{2}\left(x_{1}-x_{0}\right)^{2}-b\right)}{x_{1}-x_{0}} \ln \left(\frac{l^{2}\left(m^{2}\left(x_{1}-x_{0}\right)^{2}+b\right)}{\left(x_{1}-x_{0}\right)^{2}}\right) .
\end{aligned}
$$

The renormalized vacuum energy in region $x_{1}<x<x_{2}$ is

$$
E\left(x_{1}, x_{2}, a\right)=\frac{1}{2} \sum_{k=1}^{N-1} \varepsilon_{k}-\frac{2 l}{\pi a^{2}}+\frac{1}{2 a}+\frac{l \bar{U}\left(x_{1}, x_{2}\right)}{4 \pi} \ln \left(\frac{\chi a}{l}\right)+\frac{g\left(x_{1}, x_{2}\right)}{8 \pi l} .
$$

Fig. 9 displays this energy as a function of boundary position $x_{2}$ for fixed boundary position $x_{1}=0$. Nothing beyond the boundary at $x_{2}$ is considered here. Fig. 9 


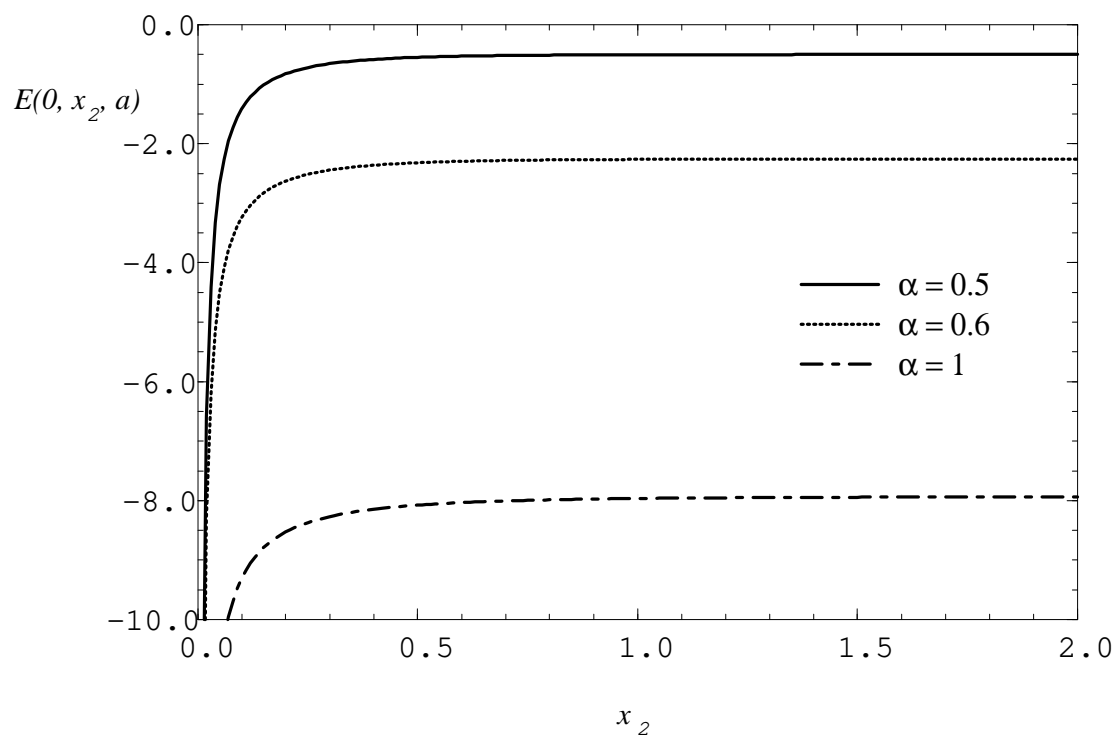

Figure 9: $E\left(x_{1}, x_{2}, a\right)$ from eq. (4.25) with $x_{0}=-0.01, m=2, a=1 / 500$ for Dirichlet boundaries at $x_{1}=0$ and variable $x_{2}$.

qualitatively resembles fig. 1 giving the comparable vacuum energy for $V(x)=0$. As $x_{2}$ approaches $x_{2}=0$ here (the boundaries $x_{1}=0$ and $x_{2}$ move close together) the function $E\left(0, x_{2}, a\right)$ approaches the function $-\pi / 24 l$ with $l=x_{2}-x_{1}$. This is the $l \rightarrow 0$ limiting form of the vacuum energy for $V(x)=0$ and $m \geq 0$ as it should be.

\subsubsection{Casimir forces}

Now we proceed to the numerical evaluation of Casimir forces for various configurations. It will be extremely important to verify that we obtain locally from $\left\langle T^{11}\right\rangle$ and globally from the vacuum energy the same Casimir forces. If this is what is found and it is - then because the local and global mathematics differ quite substantially we can be confident both are working as they should. Indeed these calculations provide the crucial test of our lattice methods with nonzero background potential $V(x)$.

Global calculations of the vacuum energy will be done using the renormalized formula (4.25). Positioning three Dirichlet boundaries as before at $x_{1}<x_{2}<x_{3}$ we obtain the Casimir force on boundary $x_{2}$ (globally) as

$$
F_{C a s}=\lim _{a \rightarrow 0}\left(\frac{E\left(x_{1}, x_{2}, a\right)-E\left(x_{1}, x_{2}+a, a\right)+E\left(x_{2}, x_{3}, a\right)-E\left(x_{2}-a, x_{3}, a\right)}{a}\right) .
$$

Alternatively $F_{\text {cas }}$ can be calculated (locally) as

$$
F_{\text {Cas }}=\lim _{a \rightarrow 0}\left(\left\langle T^{11}\right\rangle_{\text {reg }}\left(x_{1}, x_{2}, x_{2}, a\right)-\left\langle T^{11}\right\rangle_{\text {reg }}\left(x_{2}, x_{3}, x_{2}, a\right)\right) .
$$




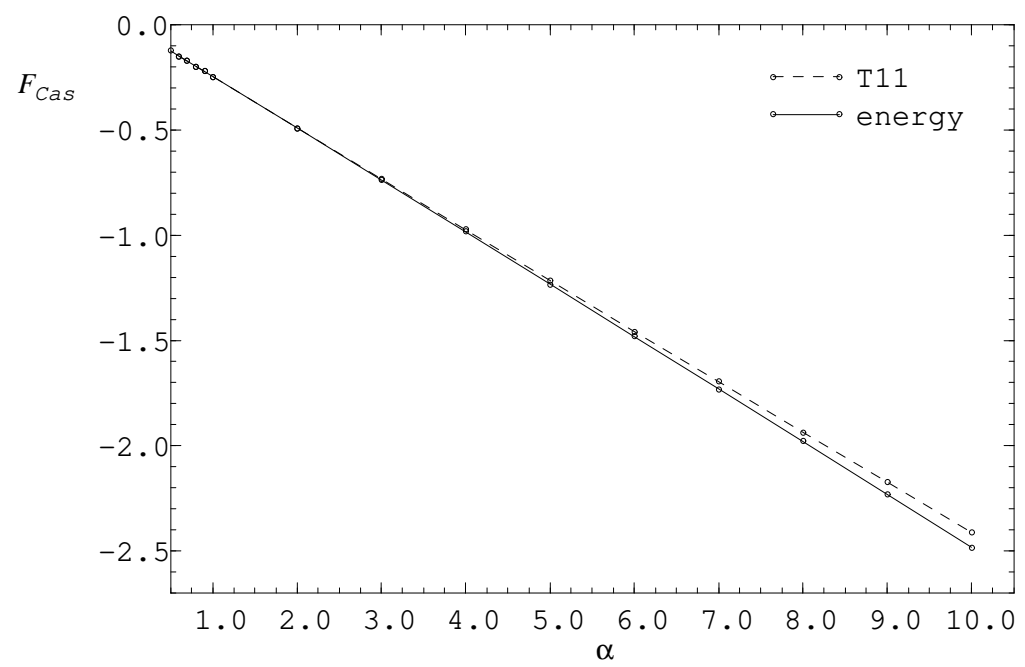

Figure 10: Casimir force on the middle boundary $x_{2}=1$ obtained globally and locally from eqs. (4.26), (4.27) with $x_{0}=-0.01, m=0, a=1 / 300$ and $x_{1}=0, x_{3}=6$.

Fig. 10 shows these two results for $F_{C a s}$ for fixed $x_{1}, x_{2}, x_{3}$ as a function of Bessel parameter $\alpha$. The agreement is very good. Let us examine this in greater detail. For small $\alpha$ we find very close agreement between the local and global calculations even for relatively large lattice constant $a$. For example, in fig. 10 at $\alpha=1$ the global and local values are -0.246007 and -0.246225 respectively. The limiting value of $F_{\text {Cas }}$ for $\alpha \rightarrow 1 / 2$ is $-\pi / 24+\pi / 600$.

As $\alpha$ increases the agreement between global and local becomes less good. To improve the agreement one must go to smaller lattice constant $a$. This is demonstrated in fig. 11 where, for $\alpha=10$, we plot the globally and locally obtained results for $F_{C a s}$ versus $a$. Linear extrapolation of the two straight lines to $a=0$ yields the limiting values -2.45142 and -2.45105 respectively. This is extremely good agreement.

Note that in fig. 10 the $(m=0)$-Casimir force is seen to depend linearly on $\alpha$. For $m>0$ this is not the case in general; however linear behavior does set in for sufficiently large $\alpha$.

To display the agreement between the global and local methods in even more detail let us decompose $F_{C a s}$ on the $x_{2}$-boundary into left $(L)$ and right $(R)$ components

$$
F_{C a s}=F_{L}+F_{R} .
$$

$F_{L}\left(F_{R}\right)$ is the force exerted on the boundary $x_{2}$ from left (right)

$$
\begin{gathered}
F_{L} \equiv-\frac{\partial E\left(x_{1}, x_{2}\right)}{\partial x_{2}}=\left\langle T^{11}\right\rangle\left(x_{1}, x_{2}, x=x_{2}\right), \\
F_{R} \equiv-\frac{\partial E\left(x_{2}, x_{3}\right)}{\partial x_{2}}=-\left\langle T^{11}\right\rangle\left(x_{2}, x_{3}, x=x_{2}\right) .
\end{gathered}
$$




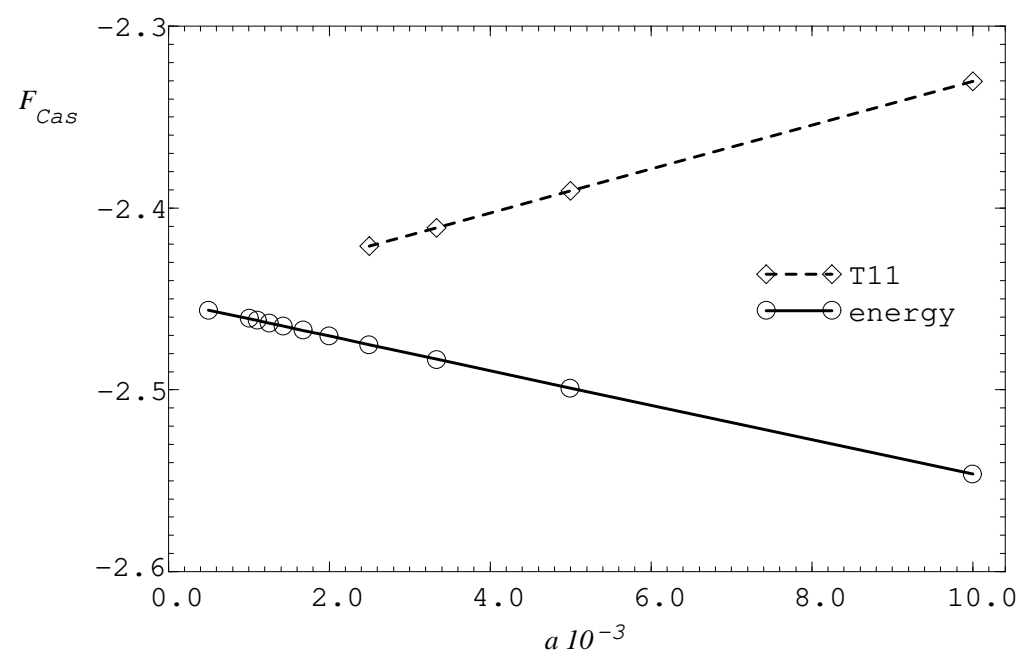

Figure 11: The global and local Casimir forces in fig. 10 for $\alpha=10$ plotted versus lattice constant $a$.

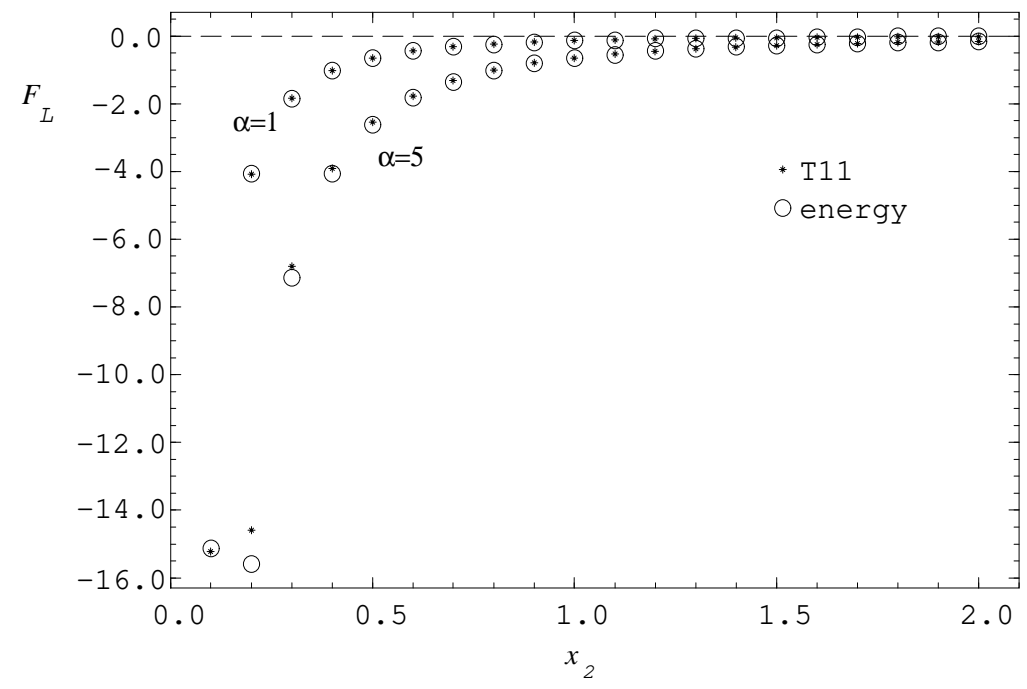

Figure 12: The left-hand Casimir forces (4.29), (4.30) on the middle wall, the latter's position $x_{2}$ being variable. Here $x_{0}=-0.01, m=1, a=$ $1 / 400$ and $x_{1}=0$. 


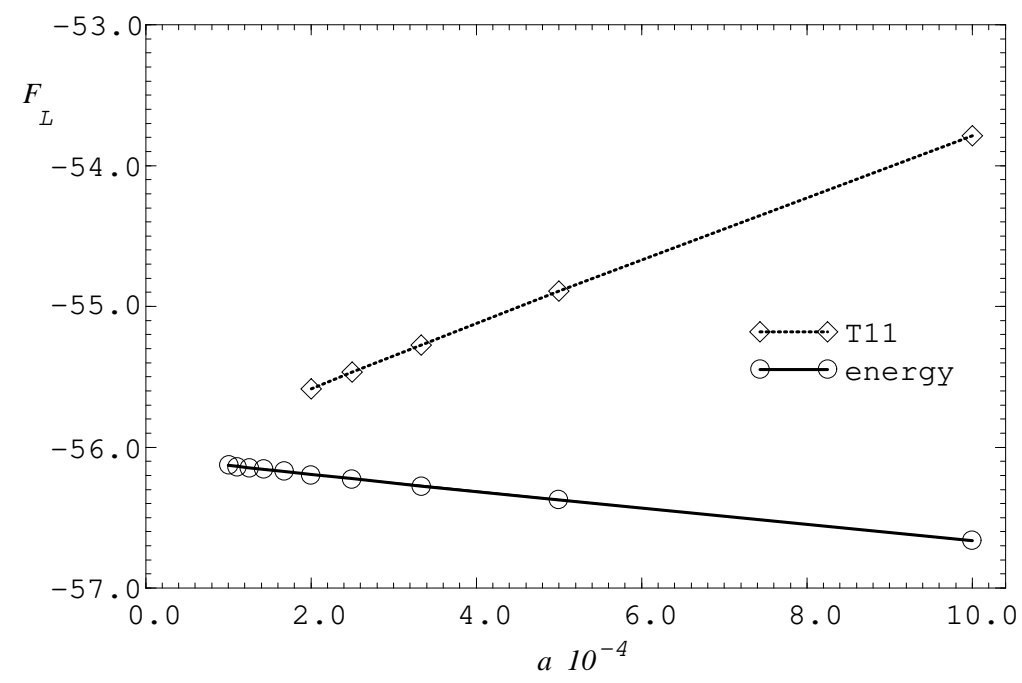

Figure 13: The left-hand Casimir forces in fig. 12 for $\alpha=5$ and $x_{2}=0.1$ plotted versus lattice constant $a$.

In fig. 12 we plot $F_{L}$ computed locally using $\left\langle T^{11}\right\rangle\left(x_{1}, x_{2}, x_{3}, a\right)$ and globally using

$$
F_{L}\left(x_{1}, x_{2}\right) \equiv-\frac{E\left(x_{1}, x_{2}+a, a\right)-E\left(x_{1}, x_{2}, a\right)}{a} .
$$

Again we find very good agreement as long as $\alpha$ is not too large and $x_{2}$ not too small. For large $\alpha$ and small $x_{2}$ the influence of $V(x)$ is quite strong and we have to go to smaller lattice constant to observe the local-global agreement. We show in fig. 13 how this works for $\alpha=5$ and $x_{2}=0.1$. Linear extrapolation to $a=0$ in fig. 13 yields the global and local values -56.073 and -56.026 respectively for $F_{L}$; again the agreement is extremely close.

The final three figures in this section show Casimir forces computed globally using the vacuum energy. This is simpler than working with $\left\langle T^{11}\right\rangle$ which requires knowledge of eigenvalues and eigenvectors. However we have verified that $\left\langle T^{11}\right\rangle$ provides the same results.

Fig. 14 shows how changing the position $x_{0}$ of the center of the Bessel boundary affects the Casimir force on boundary $x_{2}$ in the system with three Dirichlet boundaries at $x_{1}, x_{2}, x_{3}$, all embedded in the potential $V(x)=\left(\alpha^{2}-1 / 4\right) /\left(x-x_{0}\right)^{2} . F_{C a s}$ in fig. 14 is the net Casimir force on boundary $x_{2}$ with $x_{1}, x_{3}$ fixed and variable $x_{0}$. Three different $x_{2}$-values are used. Essentially in fig. 14 we are holding all three Dirichlet boundaries fixed and rigidly translating $V(x)$ by changing $x_{0}$. Clearly $F_{C a s}$ depends more sensitively on $x_{0}$ as $\alpha$ increases, which one would expect. Moreover the sensitivity of $F_{\text {Cas }}$ to $x_{0}$ increases as the distance $x_{2}-x_{1}$ between the leftmost and middle Dirichlet boundaries decreases. This is also to be expected. The conclusion is, that one has to choose $x_{0}$ very small in order to obtain relevant results for $F_{C a s}$. Our Choice $x_{0}=-0.01$ seems to be appropriate.

Finally we investigate the dependence on $x_{3}$ of the Casimir force $F_{C a s}$ on boundary 


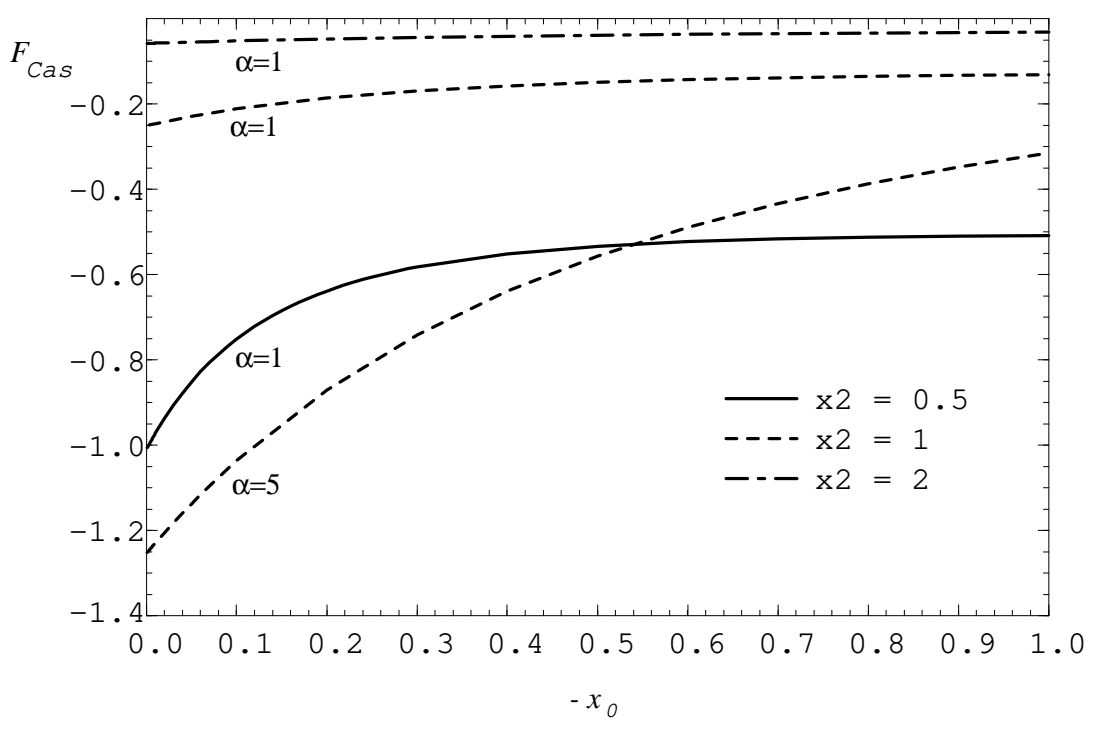

Figure 14: The Casimir force (4.26) as a function of the position $x_{0}$ of the Bessel boundary for $x_{2}=0.5,1,2$. Here $a=1 / 500, m=0, x_{1}=0$ and $x_{3}=6$.

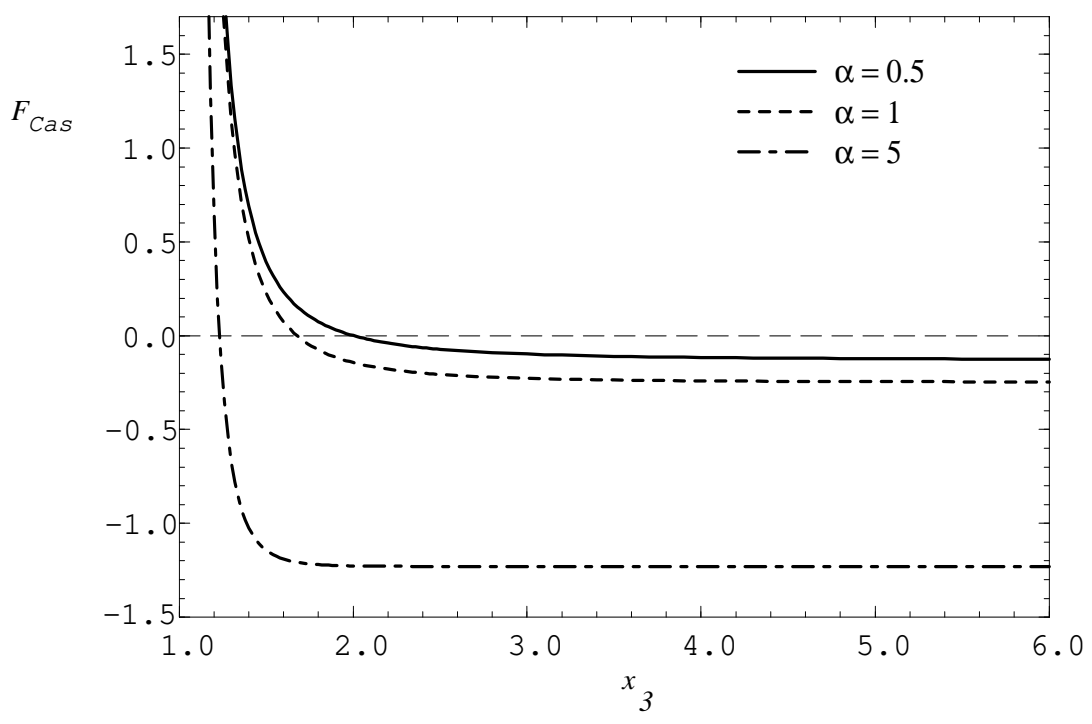

Figure 15: Global Casimir force (4.26) for variable position $x_{3}$ of the rightmost Dirichlet boundary. Here $x_{0}=-0.01, m=0, a=1 / 500$ and $x_{1}=0, x_{2}=1$. The region $x>x_{3}$ is ignored. 


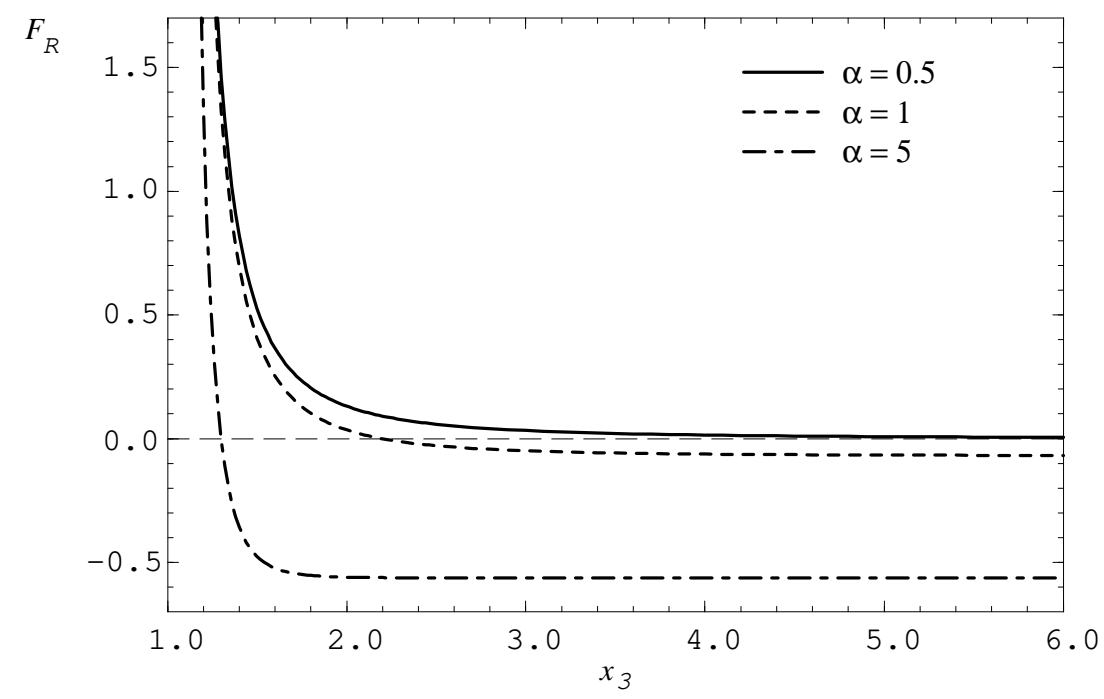

Figure 16: $F_{R}$ from eq. (4.29) for variable $x_{3}$. Same parameters as fig. 15.

$x_{2}$. For large $x_{3}$ this force (fig. 15) is practically independent of $x_{3}$ as one would expect. At relatively small $x_{3}$ a quite strong dependence on $x_{3}$ abruptly appears and $F_{C a s}$ reverses direction - again quite predictable. The value of $x_{3}$ at which $F_{C a s}$ vanishes (i.e. changes sign) moves inward with increasing $\alpha$ as it should.

Fig. 16 displays the part of the Casimir force in fig. 15 coming from $x_{2}<x<x_{3}$. For $\alpha>1 / 2$ this force component does not vanish as $x_{3} \rightarrow \infty$ (as it would if $V(x)$ were zero). The Casimir force on the Dirichlet boundary at $x_{3}$ obviously tends to zero as $x_{3} \rightarrow \infty$ because $V\left(x_{3}\right) \rightarrow 0$. Thus the asymptotic force for large $x_{3}$ in fig. 16 is entirely due to $V(x)$. Naturally this asymptotic force increases with increasing $\alpha$.

\section{Conclusion}

Continuum QFT has always been used to formulate and study the diverse topics and systems of static and dynamical Casimir theory. We decided several years ago to reformulate a broad range of these topics and systems in the language of lattice QFT, confident this would provide a tool powerful enough to solve many Casimir problems not accessible to continuum methods. Here we have presented some results on static finite lattice Casimir systems. A specific static spatial configuration was chosen: parallel Dirichlet boundaries with fixed separation on a d-dimensional lattice. We formulated this problem first with no background potential, then with a background lattice potential $V(n)$ depending on the coordinate $x=n a$ perpendicular to the boundary planes at $x=0, l$. Even for vanishing background potential $V(n)=0$ the presence of hard boundaries introduces technical features into the lattice formulation not present in lattice QFT with no boundaries. We have examined 
many of these things in detail. With the introduction of a nonzero background potential $V(n)$ there appear additional technical complexities which have also received careful attention.

Renormalization of lattice QFT in the continuum limit $a \rightarrow 0$ is a prerequisite to success. One does lattice QFT primarily to be able to learn things about the continuum theory which are difficult to find out using continuum methods. Certainly that is our attitude toward this work. Until one has understood how renormalization works one's lattice results do not mean very much: the (informationless) $a \rightarrow 0$ divergences will swamp any physical information contained in the lattice mathematics. For this reason we have paid close attention to renormalization, both for $V(n)=0$ and for $V(n)>0$. Rather detailed results have been presented for onedimensional lattices in secs. 3 and 4 . We believe these results reveal a self-consistent and clear picture of the lattice QFT as it smoothly becomes (after renormalization) the continuum theory in the limit $a \rightarrow 0$, both for vanishing and for nonvanishing background potential $V(n)$.

In unpublished work we have studied the extension of the $V(n)=0$ 1D-lattice analysis to $d \geq 2$ spatial dimensions. One can discern in the mathematics of these systems how lattice Casimir QFT in higher dimensions including renormalization parallels continuum theory. This is very important for us, because from the outset we planned to study 2D and 3D lattice configurations of hard boundaries which, because of nonsymmetric or otherwise complicated boundary geometry, cannot be dealt with using continuum mathematics. Work on such systems is in progress.

Another direction of research we are pursuing is the introduction of background potentials $V(n)$ on $d \geq 1$ spatial lattices. The semihard Bessel potential (4.2) is very useful in continuum theory because it leads to spatial modes which are known explicitly. However on the lattice it matters little whether one uses this potential or some other: The numerical work is made neither easier nor more difficult by one's choice of potential. Thus we are trying out on the lattice various semihard potentials to gain insights one would perhaps not be able to obtain differently. Another interesting type of enclosing boundary - soft boundaries - is also being studied on the lattice. We intend to report on different aspects of this work elsewhere.

Beyond the static systems mentioned we have also been able to make progress in another important area: dynamical Casimir theory. One can, on a lattice, simulate moving boundaries quite effectively. Time-dependent backgrounds excite the quantum field and one can see this clearly in one's numerical results. We shall present lattice analysis of dynamical as well as static Casimir systems in subsequent articles.

\section{Acknowledgements}

One of us (A. A) thanks the Institut für Theoretische Physik der Universität Heidelberg for its kind hospitality, and Penn State University and the LV Campus for financial support in various forms. 


\section{A Operators on the lattice}

On a lattice one can reduce any differential operator to an ordinary numerical matrix once the fields $\phi_{n}$ have been labeled and ordered. Here we consider lattice versions of the following operators

$$
(\nabla \phi)^{2}, \phi \triangle \phi \text { and } \nabla(\phi \nabla \phi)
$$

On a lattice there is, of course, more than one way to represent differentiation. We shall not be concerned here with distinguishing among different possibilities; the simplest and most direct definition will always be chosen.

\section{A.1 Laplace operator}

The lattice Laplace operator is relatively straightforward due to its symmetry. However, the precise form of the matrix representing $\triangle$ will depend on the boundary conditions imposed on the field $\phi$. One can see this from the following two simple examples.

Consider a 1D lattice having five points labeled by $i=0, \ldots, 4$ and Dirichlet boundary conditions at $i=0,4$; thus the field consists of

$$
\phi_{0}, \phi_{1}, \phi_{2}, \phi_{3}, \phi_{4}
$$

with

$$
\phi_{0}=\phi_{4}=0 \text {. }
$$

The second derivative is defined on the lattice in the usual way

$$
\left(\frac{\partial^{2} \phi}{(\partial x)^{2}}\right)_{i}=\frac{\phi_{i+1}-2 \phi_{i}+\phi_{i-1}}{a^{2}} .
$$

Because of the Dirichlet conditions we need - e.g. in eq. (2.41) - this operator only for the internal points $n_{i}(i=1,2,3)$. Thus

$$
\begin{aligned}
(\triangle \phi)_{i} & =\sum_{j=1}^{3} \triangle_{i, j} \phi_{j}, \quad i=1,2,3 \quad \text { with } \\
\triangle_{i, j} & =\frac{1}{a^{2}}\left(\delta_{i+1, j}-2 \delta_{i, j}+\delta_{i-1, j}\right), \quad i, j=1,2,3 \quad \text { or in matrix form } \\
\triangle & =\frac{1}{a^{2}}\left(\begin{array}{rrr}
-2 & 1 & 0 \\
1 & -2 & 1 \\
0 & 1 & -2
\end{array}\right)
\end{aligned}
$$

As a second example consider a 1D lattice having four points and a periodic boundary condition; thus the field consists of

$$
\phi_{1}, \phi_{2}, \phi_{3}, \phi_{4}
$$


The lattice point $n=5$ is identified with the lattice point $n=1$, i.e. $\phi_{1}=\phi_{5}$. With these boundary conditions we need - e.g. in eq. (2.41) - the second derivative also on the lattice "boundary", i.e. on the lattice point $n=1$. One finds

$$
\begin{aligned}
(\triangle \phi)_{i} & =\sum_{j=1}^{4} \triangle_{i, j} \phi_{j}, \quad i=1,2,3,4 \text { with } \\
\triangle & =\frac{1}{a^{2}}\left(\begin{array}{rrrr}
-2 & 1 & 0 & 1 \\
1 & -2 & 1 & 0 \\
0 & 1 & -2 & 1 \\
1 & 0 & 1 & -2
\end{array}\right) .
\end{aligned}
$$

Using the periodic $\delta$ function we can also write

$$
\triangle_{i, j}=\frac{1}{a^{2}}\left(\delta_{i+1, j}-2 \delta_{i, j}+\delta_{i-1, j}\right), \quad i, j=1,2,3,4 .
$$

From these two simple examples one can immediately write down a general expression for the Laplace operator appearing in eq. 2.41)

$$
\triangle_{n, n^{\prime}}=\sum_{j=1}^{d} \frac{1}{a^{2}}\left(\delta_{n+\vec{e}_{j}, n^{\prime}}-2 \delta_{n, n^{\prime}}+\delta_{n-\vec{e}_{j}, n^{\prime}}\right), \quad n, n^{\prime} \in G .
$$

Here the $\delta$-function $\delta_{n, n^{\prime}}$ is appropriately periodic. This notation is, for several dimensions, neither very transparent nor very well suited for numerical work. One can however relabel the matrix elements according to e.g. the scheme

$$
\begin{aligned}
& \left(n_{1}, \ldots, n_{d}\right) \rightarrow m \\
m= & \left(n_{1}-1\right)\left(2 N_{\perp}\right)^{d-1}+\left(n_{2}-1+N_{\perp}\right)\left(2 N_{\perp}\right)^{d-2} \\
+ & \cdots+\left(n_{d-1}-1+N_{\perp}\right)\left(2 N_{\perp}\right)+n_{d}+N_{\perp} \\
1 \leq & n_{1} \leq N_{1}-1,-N_{\perp}+1 \leq n_{i} \leq N_{\perp} \quad(i=2, \cdots, d) .
\end{aligned}
$$

This produces a symmetric matrix $\triangle_{m^{\prime}, m}$, whose dimension $\left(N_{1}-1\right)\left(2 N_{\perp}\right)^{d-1}$ equals the number of lattice points.

\section{A.2 The operators $(\nabla \phi)^{2}$ and $\nabla(\phi \nabla \phi)$}

The lattice representation of the differential operator $\nabla$ is not unique. Let us again use the periodic $1 D$ lattice with four lattice points to illustrate this. We consider three definitions of $\nabla$ :

$$
\text { (1) }(\nabla \phi)_{i}=\frac{\phi_{i+1}-\phi_{i}}{a}
$$

whose matrix representation $(\nabla \phi)_{i}=\sum_{j=1}^{4} D_{i, j} \phi_{j}$ is

$$
D_{i, j}^{(1)}=\frac{1}{a}\left(\begin{array}{rrrr}
-1 & 1 & 0 & 0 \\
0 & -1 & 1 & 0 \\
0 & 0 & -1 & 1 \\
1 & 0 & 0 & -1
\end{array}\right)=\frac{1}{a}\left(\delta_{i+1, j}-\delta_{i, j}\right),
$$




$$
\text { (2) }(\nabla \phi)_{i}=\frac{\phi_{i}-\phi_{i-1}}{a}
$$

whose matrix form is

$$
\begin{gathered}
D_{i, j}^{(2)}=-\left(D^{(1) \top}\right)_{i, j}=\frac{1}{a}\left(\begin{array}{rrrr}
1 & 0 & 0 & -1 \\
-1 & 1 & 0 & 0 \\
0 & -1 & 1 & 0 \\
0 & 0 & -1 & 1
\end{array}\right)=\frac{1}{a}\left(\delta_{i, j}-\delta_{i-1, j}\right), \\
(3) \quad(\nabla \phi)_{i}=\frac{\phi_{i+1}-\phi_{i-1}}{2 a}
\end{gathered}
$$

whose matrix form is

$$
D_{i, j}^{(3)}=\frac{1}{2 a}\left(\begin{array}{rrrr}
0 & 1 & 0 & -1 \\
-1 & 0 & 1 & 0 \\
0 & -1 & 0 & 1 \\
1 & 0 & -1 & 0
\end{array}\right)=\frac{1}{2 a}\left(\delta_{i+1, j}-\delta_{i-1, j}\right) .
$$

Definitions (1) and (2) have the disadvantage of producing unsymmetric matrices. However, it is not our purpose here to discretize $\nabla \phi$, but rather to define the lattice operator $(\nabla \phi)^{2}$.

For periodic boundary conditions one has in the continuum theory

$$
\int d x(\nabla \phi(x))^{2}=-\int d x \phi(x) \triangle \phi(x) .
$$

The analogous statement on the periodic lattice is (here $k=1,2,3$ labels the three definitions)

$$
\left.a \sum_{n}\left(\sum_{i} D_{n, i}^{(k)} \phi_{i}\right)\left(\sum_{j} D_{n, j}^{(k)} \phi_{j}\right)=a \sum_{i} \sum_{j} \phi_{i}\left(\sum_{n}\left(D^{(k) \top}\right)_{i, n} D_{n, j}^{(k)}\right) \phi_{j}\right) \equiv-a \sum_{i, j} \phi_{i} \triangle_{i, j} \phi_{j} .
$$

It should be true that

$$
\sum_{n}\left(D^{(k) \top}\right)_{i, n} D_{n, j}^{(k)}=-\triangle_{i, j} .
$$

However, when we calculate the left-hand side of this equation we find

$$
\sum_{n}\left(D^{(1) \top}\right)_{i, n} D_{n, j}^{(1)}=\sum_{n}\left(D^{(2) \top}\right)_{i, n} D_{n, j}^{(2)}=\frac{1}{a^{2}}\left(\begin{array}{rrrr}
2 & -1 & 0 & -1 \\
-1 & 2 & -1 & 0 \\
0 & -1 & 2 & -1 \\
-1 & 0 & -1 & 2
\end{array}\right)
$$

and

$$
\sum_{n}\left(D^{(3) \top}\right)_{i, n} D_{n, j}^{(3)}=\frac{1}{a^{2}}\left(\begin{array}{rrrr}
2 & 0 & -2 & 0 \\
0 & 2 & 0 & -2 \\
-2 & 0 & 2 & 0 \\
0 & -2 & 0 & 2
\end{array}\right)
$$


Since we use the lattice Laplace operator (A.7) for periodic conditions we see that eq. (A.19) is fulfilled only for the unsymmetric discretizations $k=1,2$. Thus we are led to our final (symmetric) choice of $(\nabla \phi)^{2}$

$$
\begin{aligned}
(\nabla \phi)_{n}^{2} & =\frac{1}{2}\left(\left(\frac{\phi_{n+1}-\phi_{n}}{a}\right)^{2}+\left(\frac{\phi_{n}-\phi_{n-1}}{a}\right)^{2}\right) \\
& =-\phi_{n} \frac{\phi_{n+1}-2 \phi_{n}+\phi_{n-1}}{a^{2}}+\frac{\phi_{n+1}^{2}-2 \phi_{n}^{2}+\phi_{n-1}^{2}}{2 a^{2}} \\
& =-\phi_{n} \sum_{n^{\prime}} \triangle_{n, n^{\prime}} \phi_{n^{\prime}}+\frac{\phi_{n+1}^{2}-2 \phi_{n}^{2}+\phi_{n-1}^{2}}{2 a^{2}} .
\end{aligned}
$$

Using

$$
(\nabla \phi)^{2}=\nabla(\phi \nabla \phi)-\phi \triangle \phi
$$

we obtain simultaneously from (A.22) a symmetrical definition for $\nabla(\phi \nabla \phi)$

$$
(\nabla(\phi \nabla \phi))_{n}=\frac{\phi_{n+1}^{2}-2 \phi_{n}^{2}+\phi_{n-1}^{2}}{2 a^{2}} .
$$

An important mathematical statement for periodic boundary conditions is the "spatial integral"

$$
\int_{0}^{l} d x \nabla(\phi \nabla \phi) \rightarrow \sum_{n=1}^{N} a(\nabla(\phi \nabla \phi))_{n}=0 .
$$

With this lattice definition of $\int_{0}^{l} d x$ eq. (A.17) is satisfied on the periodic lattice. Eqs. (A.22), (A.24) are also applicable for Dirichlet boundary conditions as long as one stays away from the boundaries. Boundary points have to be dealt with separately. For the left and right boundary points $n=0, N$ one can define rightand left-handed derivatives as follows

$$
\begin{aligned}
(\nabla \phi)_{0}^{2} & =(\nabla(\phi \nabla \phi))_{0}=\frac{1}{a^{2}} \phi_{1}^{2} \\
(\nabla \phi)_{N}^{2} & =(\nabla(\phi \nabla \phi))_{N}=\frac{1}{a^{2}} \phi_{N-1}^{2} .
\end{aligned}
$$

Then we can also use eqs. (A.22), (A.24) at Dirichlet boundary points if we adopt the definitions

$$
\begin{aligned}
\phi_{-1}^{2} & \equiv \phi_{1}^{2}, \\
\phi_{N+1}^{2} & \equiv \phi_{N-1}^{2} .
\end{aligned}
$$

In the massive theory without potential we need more specifically $\phi_{-1}=-\phi_{1}$, $\phi_{N+1}=-\phi_{N-1}$.

Including eqs. A.28), (A.29) we are led to a mathematical statement parallel to eq. 
(A.25), but for Dirichlet boundaries

$$
\begin{aligned}
& \frac{1}{2}\left(\sum_{n=1}^{N} a(\nabla(\phi \nabla \phi))_{n}+\sum_{n=0}^{N-1} a(\nabla(\phi \nabla \phi))_{n}\right)= \\
& \quad=\sum_{n=1}^{N-1} a(\nabla(\phi \nabla \phi))_{n}+\frac{1}{2 a^{2}}(\nabla(\phi \nabla \phi))_{N}+\frac{1}{2 a^{2}}(\nabla(\phi \nabla \phi))_{0} \\
& \quad=-\frac{1}{2 a^{2}} \phi_{1}^{2}-\frac{1}{2 a^{2}} \phi_{N-1}^{2}+\frac{1}{2 a^{2}} \phi_{N-1}^{2}+\frac{1}{2 a^{2}} \phi_{1}^{2} \\
& \quad=0 .
\end{aligned}
$$

Thus the appropriate lattice definition of "spatial integral" for Dirichlet boundaries is

$$
\int_{0}^{l} d x \rightarrow \frac{1}{2} a\left(\sum_{n=1}^{N}+\sum_{n=0}^{N-1}\right)
$$

and eq. (A.17) is again satisfied.

In summary: For the basic lattice of this paper with Dirichlet boundaries at $x_{1}=0, l$ and $d-1$ periodic directions we have, with eq. (A.9)

$$
\begin{gathered}
(\nabla \phi)_{n}^{2}=-\phi_{n} \sum_{n^{\prime} \in G} \triangle_{n, n^{\prime}} \phi_{n^{\prime}}+\sum_{j=1}^{d} \frac{\phi_{n+\vec{e}_{j}}^{2}-2 \phi_{n}^{2}+\phi_{n-\vec{e}_{j}}^{2}}{2 a^{2}} \\
(\nabla(\phi \nabla \phi))_{n}=\sum_{j=1}^{d} \frac{\phi_{n+\vec{e}_{j}}^{2}-2 \phi_{n}^{2}+\phi_{n-\vec{e}_{j}}^{2}}{2 a^{2}} .
\end{gathered}
$$

\section{B A lattice artefact}

In calculating the $(d=1)$-vacuum stress tensor in sec. 3 with $V(x)=0$ we encountered a lattice artefact in the lattice formulation which (i) diverges in the continuum limit $a \rightarrow 0$ and (ii) has no counterpart in the continuum theory. The origin of such a lattice quantity is to be found in the noncommutativity of the mode sum over "momentum" $k$ with the limit $a \rightarrow 0$. Our task in this appendix will be to establish some needed properties of this lattice artefact term

$$
A(m, l, n, a)=-\sum_{k=1}^{N-1} \frac{2 N^{2} \sin ^{4}\left(\frac{\pi k}{2 N}\right) \cos \left(\frac{2 \pi k n}{N}\right)}{l^{2} \sqrt{(m l)^{2}+4 N^{2} \sin ^{2}\left(\frac{\pi k}{2 N}\right)}} \text { with } N=\frac{l}{a}
$$

Most importantly we will show that $A$ contains no physical information.

To illustrate noncommutativity let us observe that

$$
\lim _{a \rightarrow 0} A(m, l, n, a) \neq 0
$$


while in the reverse order

$$
\sum_{k=1}^{\infty} \lim _{a \rightarrow 0}\left(\frac{2 N^{2} \sin ^{4}\left(\frac{\pi k}{2 N}\right) \cos \left(\frac{2 \pi k n}{N}\right)}{l^{2} \sqrt{(m l)^{2}+N^{2} \sin ^{2}\left(\frac{\pi k}{2 N}\right)}}\right)=0 .
$$

Note that $A$ has no global counterpart

$$
\sum_{n=1}^{N} A(m, l, n, a)=0
$$

because $\sum_{n=1}^{N} \cos (2 \pi k n / N)=0$ for $k \neq 0$. Expanding $A$ in powers of $m^{2}$ we find

$$
\begin{aligned}
A(m, l, n, a)= & -\frac{1}{a l} \sum_{k=1}^{N-1} \sin ^{3}\left(\frac{\pi k}{2 N}\right) \cos \left(\frac{2 \pi k n}{N}\right) \\
& +\frac{m^{2}}{8} \sum_{k=1}^{N-1} \frac{1}{N} \sin \left(\frac{\pi k}{2 N}\right) \cos \left(\frac{2 \pi k n}{N}\right) \\
& -\frac{1}{l^{2}} \sum_{\nu=2}^{\infty} c_{\nu}\left(\frac{m l}{2}\right)^{2 \nu} \sum_{k=1}^{N-1} \frac{\cos \left(\frac{2 \pi k n}{N}\right)}{N^{2}\left(N \sin \left(\frac{\pi k}{2 N}\right)\right)^{2 \nu-3}} \\
= & I+I I+I I I .
\end{aligned}
$$

The term III:

One can verify (numerically for example) that for $\operatorname{Re} \mu>-1$

$$
\lim _{N \rightarrow \infty}\left(\sum_{k=1}^{N-1} \frac{\cos \left(\frac{2 \pi k n}{N}\right)}{N^{2}\left(N \sin \left(\frac{\pi k}{2 N}\right)\right)^{\mu}}\right)=0, \quad \forall n, \operatorname{Re} \mu>-1 .
$$

In the contribution III we need this statement for $\mu=2 \nu-3 \geq 1$. To illustrate the limit (B.6) consider the $\mu=1$ case. Because

$$
\sin (x) \geq \frac{2}{\pi} x, \quad 0 \leq x \leq \frac{\pi}{2},
$$

it follows that

$$
\sum_{k=1}^{N-1} \frac{\cos \left(\frac{2 \pi k n}{N}\right)}{N^{2} N \sin \left(\frac{\pi k}{2 N}\right)} \leq \frac{1}{N^{2}} \sum_{k=1}^{N-1} \frac{1}{k} \leq \frac{1}{N}=\mathcal{O}(a) \quad \forall n .
$$

For $\mu>1$

$$
s k \frac{\cos \left(\frac{2 \pi k n}{N}\right)}{N^{2}\left(N \sin \left(\frac{\pi k}{2 N}\right)\right)^{\mu}}=\mathcal{O}\left(a^{2}\right) \quad \forall n, \mu>1
$$

because the sum over $k$ now converges. In summary, the contribution III is at most $\mathcal{O}(a)$ and therefore this contribution to $A$ vanishes in the continuum limit. 
The term II:

We define

$$
\sum_{k=1}^{N-1} \frac{1}{N} \sin \left(\frac{\pi k}{2 N}\right) \cos \left(\frac{2 \pi k n}{N}\right)=: h\left(\frac{l}{a}, n\right) .
$$

Clearly

$$
\sum_{n=1}^{N} h\left(\frac{l}{a}, n\right)=0
$$

The finite sum $(\mathbb{B . 1 0})$ can be evaluated in closed form

$$
h\left(\frac{l}{a}, n\right)=\frac{a}{4 l}\left(\frac{1}{\tan \left(\frac{\pi a}{4 l}+\pi \frac{n a}{l}\right)}+\frac{1}{\tan \left(\frac{\pi a}{4 l}-\pi \frac{n a}{l}\right)}-2\right) .
$$

Writing $n=x / a$ and using the formula

$$
\frac{1}{\tan (a+x)}+\frac{1}{\tan (a-x)}=-2\left(1+\frac{1}{\tan ^{2} x}\right) a+\mathcal{O}\left(a^{3}\right) \quad \text { für } \quad a \ll x
$$

we obtain

$$
h\left(\frac{l}{a}, \frac{x}{a}\right)=-\frac{a}{2 l}+\mathcal{O}\left(a^{2}\right), \quad \frac{a}{4} \ll x \ll\left(l-\frac{a}{4}\right) .
$$

In the continuum limit

$$
\lim _{a \rightarrow 0} h\left(\frac{l}{a}, \frac{x}{a}\right)=0, \quad 0<x<l
$$

and therefore

$$
\lim _{a \rightarrow 0} h\left(\frac{l}{a}, \frac{x}{a}\right)=\sum_{k=1}^{\infty} \lim _{a \rightarrow 0}\left(\frac{1}{N} \sin \left(\frac{\pi k}{2 N}\right) \cos \left(\frac{2 \pi k n}{N}\right)\right)=0, \quad 0<x<l .
$$

For $0<x<l$ it does not matter how one takes the limit $a \rightarrow 0$. However on the boundaries $x=0, l$ we have

$$
h\left(\frac{l}{a}, 0\right)=h\left(\frac{l}{a}, N\right)=\frac{a}{2 l}\left(\frac{1}{\tan \left(\frac{\pi a}{4 l}\right)}-1\right) \stackrel{a \rightarrow 0}{\longrightarrow} \frac{2}{\pi} .
$$

In other words, the $(N \rightarrow \infty)$-limit of the term II is nonuniform in lattice position $n$. This phenomenon is quite familiar in infinite series. We see in eq. (B.12) that $h$ has poles at $n=1 / 4$ and $n=N-1 / 4$. These poles lie between the boundary and the first adjacent lattice points - i.e. they are not on the lattice. They signal to us that the lattice function $h$ is nonuniform as the boundaries are reached.

According to eq. (B.16) the continuum limit of II is zero between the Dirichlet endpoints. At these endpoints the value of II in the continuum limit jumps to 
$m^{2} / 4 \pi$. As there is no $l$ dependence in this contribution to $A$ one sees that the Casimir force is unaffected.

The term I:

We define

$$
-\frac{1}{a l} \sum_{k=1}^{N-1} \sin ^{3}\left(\frac{\pi k}{2 N}\right) \cos \left(\frac{2 \pi k n}{N}\right)=: f(l, n, a)+\frac{1}{2 a l}
$$

where the function $f$ can also be written

$$
f(l, n, a)=-\frac{1}{l^{2}} \sum_{k=1}^{N-1}\left(N \sin ^{3}\left(\frac{\pi k}{2 N}\right) \cos \left(\frac{2 \pi k n}{N}\right)+\frac{k}{N-1}\right) .
$$

The finite series (B.18) can be evaluated in closed form

$$
\begin{aligned}
& -\frac{1}{a l} \sum_{k=1}^{N-1} \sin ^{3}\left(\frac{\pi k}{2 N}\right) \cos \left(\frac{2 \pi k n}{N}\right)=\frac{1}{2 a l} \\
& +\frac{1}{16 a l}\left(\frac{1}{\tan \left(\frac{3 \pi}{4 N}+\pi \frac{n}{N}\right)}+\frac{1}{\tan \left(\frac{3 \pi}{4 N}-\pi \frac{n}{N}\right)}-\frac{3}{\tan \left(\frac{\pi}{4 N}+\pi \frac{n}{N}\right)}-\frac{3}{\tan \left(\frac{\pi}{4 N}-\pi \frac{n}{N}\right)}\right)
\end{aligned}
$$

and

$$
\begin{array}{r}
f(l, n, a)=\frac{1}{16 a l}\left(\frac{1}{\tan \left(\frac{3 \pi a}{4 l}+\pi \frac{n a}{l}\right)}+\frac{1}{\tan \left(\frac{3 \pi a}{4 l}-\pi \frac{n a}{l}\right)}\right. \\
\left.-\frac{3}{\tan \left(\frac{\pi a}{4 l}+\pi \frac{n a}{l}\right)}-\frac{3}{\tan \left(\frac{\pi a}{4 l}-\pi \frac{n a}{l}\right)}\right) .
\end{array}
$$

We see from eq. (B.21) that $f(l, n, a)$ has poles at $n=1 / 4,3 / 4$ and $n=l-1 / 4, l-$ $3 / 4$. These poles lie between the Dirichlet boundaries and their first adjacent lattice points. From this we anticipate that the function $f$ will also exhibit nonuniform behavior as the boundaries are reached.

From eq. (B.13) it follows that

$$
f\left(l, \frac{x}{a}, a\right)=\mathcal{O}\left(a^{2}\right), \quad \frac{3 a}{4} \ll x \ll l-\frac{3 a}{4} .
$$

This means that $f$ vanishes in the limit $a \rightarrow 0$ everywhere between the boundaries

$$
\lim _{a \rightarrow 0} f\left(l, \frac{x}{a}, a\right)=0, \quad 0<x<l,
$$

and that for $0<x<l$ we can in eq. (B.19) freely interchange $\sum_{k}$ with $(a \rightarrow 0)$. The behavior of $f$ on the boundary is obtained from the expansion (for $n \ll N$ )

$$
f(l, n, a)=\frac{\pi}{4 a^{2}}\left(\frac{1}{3+4 n}+\frac{1}{3-4 n}-\frac{3}{1+4 n}-\frac{3}{1-4 n}\right)+\mathcal{O}\left(a^{2}\right), \quad n \ll N .
$$

In the limit $a \rightarrow 0$ this expression diverges; however the value approached is independent of $l$. The Casimir force on the boundary is the discontinuity in $\left\langle T^{11}\right\rangle$ 
across the boundary. An $l$-independent jump in $\left\langle T^{11}\right\rangle$ makes no contribution to the Casimir force. On the boundary $\sum_{k}$ and $a \rightarrow 0$ are not interchangeable. Note also that

$$
\sum_{n=1}^{N} a f(l, n, a)=-\frac{1}{2 a}
$$

In summary we have for the lattice artefact the following:

$$
\begin{aligned}
A(m, l, n, a) & =\frac{1}{2 a l}+f(l, n, a)+\frac{m^{2}}{8} h(l, n, a)+\mathcal{O}(a), \\
\sum_{n=1}^{N} A(m, l, n, a) & =0 .
\end{aligned}
$$

$A(m, n, l, a)-1 /(2 a l)$ vanishes in the continuum limit $a \rightarrow 0$ for every point between the Dirichlet boundaries. This function on the boundaries $x=0, l$ jumps to a nonzero value which - being independent of $l$ - cannot influence the Casimir force. The comment now to follow is speculative and mathematical. Let us define a lattice function

$$
\begin{array}{r}
\delta(l, n, a):=-2 a f(l, n, a)=\frac{1}{8 l}\left(-\frac{1}{\tan \left(\frac{3 \pi a}{4 l}+\pi \frac{n a}{l}\right)}-\frac{1}{\tan \left(\frac{3 \pi a}{4 l}-\pi \frac{n a}{l}\right)}\right. \\
\left.+\frac{3}{\tan \left(\frac{\pi a}{4 l}+\pi \frac{n a}{l}\right)}+\frac{3}{\tan \left(\frac{\pi a}{4 l}-\pi \frac{n a}{l}\right)}\right)
\end{array}
$$

which seems to be rather like a $\delta$ function. Eq. (B.25) now reads

$$
\sum_{n=1}^{N} a \delta(l, n, a)=1 .
$$

The properties of $f$ discussed above show that

$$
\delta(l, n, a)= \begin{cases}O(a) & 0<n<l \\ O(1 / a) & n=0, l\end{cases}
$$

and the "integration" (B.27) over the lattice only receives a contribution from the boundaries. This suggests that

$$
\lim _{a \rightarrow 0} \delta(l, n, a)=\frac{1}{2}(\delta(x)+\delta(x-l)) .
$$

To test this numerically we applied a test-function polynomial $t(x)=A x^{3}+B x^{2}+$ $C x+D$ and found that, with very high accuracy

$$
\begin{aligned}
\frac{1}{2}\left(\sum_{n=1}^{N} a \delta(l, n, a) t(n a)+\sum_{n=0}^{N-1} a \delta(l, n, a) t(n a)\right) & \approx \frac{1}{2}(t(0)+t(l)), \\
\sum_{n=n_{1}(a)}^{n_{2}(a)} a \delta(l, n, a) t(n a) & \approx 0 .
\end{aligned}
$$


In the latter sum, for each lattice constant $a$ used we required that

$$
n_{1}(a) a=\text { const }=x_{1}>0 \quad \text { and } \quad x_{1}<n_{2}(a) a=\text { const }=x_{2}<l .
$$

Finally we mention that

$$
\begin{aligned}
& \delta_{1}(l, n, a) \equiv \frac{1}{2 l}\left(\frac{1}{\tan \left(\frac{\pi a}{4 l}+\pi \frac{n a}{l}\right)}+\frac{1}{\tan \left(\frac{\pi a}{4 l}-\pi \frac{n a}{l}\right)}\right), \\
& \delta_{2}(l, n, a) \equiv-\frac{1}{2 l}\left(\frac{1}{\tan \left(\frac{3 \pi a}{4 l}+\pi \frac{n a}{l}\right)}+\frac{1}{\tan \left(\frac{3 \pi a}{4 l}-\pi \frac{n a}{l}\right)}\right)
\end{aligned}
$$

have the same properties as $\delta(l, n, a)$. Also

$$
\delta(l, n, a)=\frac{3}{4} \delta_{1}(l, n, a)+\frac{1}{4} \delta_{2}(l, n, a) .
$$

\section{Perturbation theory on the one-dimensional lat- tice}

Here we calculate to first order in the lattice potential $U(n)=m^{2}+V(n)$ the lattice vacuum energy $E_{v a c}$ and stress tensor component $\left\langle T^{11}\right\rangle$ for the one-dimensional lattice with Dirichlet conditions at its endpoints. The "unperturbed" system is the massless scalar field with vanishing background potential $V(n)=0$. Our calculation for arbitrary $U(n)$ will determine the additional divergences in $E_{v a c}$ and $\left\langle T^{11}\right\rangle$ arising from $U(n)$.

For a given lattice potential one begins by first determining numerically the eigenvectors and spectrum of the matrix

$$
\begin{aligned}
\mathcal{O}_{n, n^{\prime}}(\lambda) & =\triangle_{n, n^{\prime}}+\lambda U(n) \delta_{n, n^{\prime}} \\
& =-\frac{\delta_{n+1, n^{\prime}}-2 \delta_{n, n^{\prime}}+\delta_{n-1, n^{\prime}}}{a^{2}}+\lambda U(n) \delta_{n, n^{\prime}}, \quad 1 \leq n, n^{\prime} \leq N-1 .
\end{aligned}
$$

Then from these ingredients one can find the first-order shift in the vacuum energy and in $\left\langle T^{11}\right\rangle$.

For $\lambda=0$ the spectrum $\omega_{k}^{2}$ and eigenvectors $\vec{w}^{k}$ of (C.1) are known

$$
\begin{aligned}
\omega_{k}^{2} & =\frac{4}{a^{2}} \sin ^{2}\left(\frac{\pi k}{2 N}\right), \\
w_{n}^{k} & =\sqrt{\frac{2}{N}} \sin \left(\frac{\pi k n}{N}\right),
\end{aligned}
$$

where $1 \leq n \leq N-1$ and $l=N a$. These eigenvalues are not degenerate, so we can use nondegenarate perturbation theory. 
For $\lambda \neq 0$ we name the eigenvalues and eigenvectors of (C.1) $\varepsilon_{k}^{2}(\lambda)$ and $\vec{v}^{k}(\lambda)$ respectively

$$
\sum_{n^{\prime}=1}^{N-1} \mathcal{O}_{n, n^{\prime}}(\lambda) v_{n^{\prime}}^{k}(\lambda)=\varepsilon_{k}^{2}(\lambda) v_{n}^{k}(\lambda) .
$$

Expansion in powers of $\lambda$ yields (see e.g. ref. [13])

$$
\begin{aligned}
\varepsilon_{k}^{2}(\lambda) & =\omega_{k}^{2}+\lambda \varepsilon_{k}^{2(1)}+\lambda^{2} \varepsilon_{k}^{2(2)}+\cdots, \\
\vec{v}^{k}(\lambda) & =\vec{w}^{k}+\lambda \vec{v}^{k(1)}+\lambda^{2} \vec{v}^{k(2)}+\cdots
\end{aligned}
$$

where

$$
\begin{aligned}
& \varepsilon_{k}^{2(1)}=\left(\vec{w}^{k} U \vec{w}^{k}\right)=\sum_{n=1}^{N-1} \frac{2}{N} \sin ^{2}\left(\frac{\pi k n}{N}\right) U(n) \\
& \stackrel{a \rightarrow 0}{\longrightarrow} \frac{2}{l} \int_{0}^{l} \sin ^{2}\left(\frac{\pi k x}{l}\right) U(x) d x, \\
& \vec{v}^{k(1)=} \sum_{\substack{p=1 \\
p \neq k}} \frac{\left(\vec{w}^{p} U \vec{w}^{k}\right)}{\omega_{k}^{2}-\omega_{p}^{2}} \vec{w}^{p} .
\end{aligned}
$$

Ground state energy:

In the expansion of the lattice vacuum energy

$$
E(\lambda)=\sum_{k=1}^{N-1} \frac{1}{2} \varepsilon_{k}(\lambda)=E^{(0)}+\lambda E^{(1)}+\lambda^{2} E^{(2)}+\cdots .
$$

the leading unperturbed term is

$$
E^{(0)}=\frac{1}{2} \sum_{k=1}^{N-1} \omega_{k}=\frac{2 l}{\pi a^{2}}-\frac{1}{2 a}-\frac{\pi}{24 l}+\mathcal{O}\left(a^{2}\right) .
$$

To find the first correction to this we need the connection between the terms in

$$
\varepsilon_{k}(\lambda)=\sqrt{\varepsilon_{k}^{2}(\lambda)}=\omega_{k}+\lambda \varepsilon_{k}^{(1)}+\lambda^{2} \varepsilon_{k}^{(2)}+\cdots
$$

and the terms in eq. (C.5). From the identity

$$
\begin{aligned}
\left(\sum_{\nu=0}^{\infty} a_{\nu} x^{\nu}\right)^{\frac{1}{2}} & =\sum_{\nu=0}^{\infty} b_{\nu} x^{\nu} \\
\Rightarrow \quad \sum_{\nu=0}^{\infty} a_{\nu} x^{\nu} & =\sum_{\nu=0}^{\infty} b_{\nu} x^{\nu} \sum_{\mu=0}^{\infty} b_{\mu} x^{\mu}=\sum_{\nu=0}^{\infty}\left(\sum_{\mu=0}^{\nu} b_{\nu-\mu} b_{\mu}\right) x^{\nu}
\end{aligned}
$$

one finds

$$
a_{\nu}=\sum_{\mu=0}^{\nu} b_{\nu-\mu} b_{\mu} .
$$


Thus

$$
\begin{array}{ll}
\nu=0: & b_{0}=\sqrt{a_{0}} . \\
\nu=1: & b_{1}=\frac{a_{1}}{2 \sqrt{a_{0}}} . \\
\nu=2: & b_{2}=\frac{1}{2 \sqrt{a_{0}}}\left(a_{2}-\frac{a_{1}^{2}}{4 a_{0}}\right) .
\end{array}
$$

Then from

$$
\varepsilon_{k}^{(1)}=\frac{\varepsilon_{k}^{2(1)}}{2 \omega_{k}}=\frac{\left(\vec{w}^{k} U \vec{w}^{k}\right)}{2 \omega_{k}}
$$

we find

$$
E^{(1)}=\sum_{k=1}^{N-1} \frac{1}{2} \varepsilon_{k}^{(1)}=\frac{l}{8} \sum_{k=1}^{N-1} \frac{1}{N \sin \left(\frac{\pi k}{2 N}\right)} \sum_{n=1}^{N-1} \frac{2}{N} \sin ^{2}\left(\frac{\pi k n}{N}\right) U(n) .
$$

This is the first-order correction to the lattice vacuum energy for arbitrary lattice potential $V(n)$.

Let us define

$$
U(x)=\bar{U}+\widehat{U}(x)
$$

with

$$
\begin{gathered}
\bar{U} \equiv \frac{1}{l} \int_{0}^{l} U(x) d x \\
\int_{0}^{l} \widehat{U}(x) d x=0
\end{gathered}
$$

Substitution in eq. (C.16) yields with the help of (3.15)

$$
\begin{aligned}
E^{(1)} & =\frac{1}{8} \int_{0}^{l} U(x) d x \sum_{k=1}^{N-1} \frac{1}{N \sin \left(\frac{\pi k}{2 N}\right)}+\frac{l}{8} \sum_{k=1}^{N-1} \frac{1}{N \sin \left(\frac{\pi k}{2 N}\right)} \sum_{n=1}^{N-1} \frac{2}{N} \sin ^{2}\left(\frac{\pi k n}{N}\right) \widehat{U}(n) \\
& =\frac{1}{8} \int_{0}^{l} U(x) d x\left(\frac{2}{\pi} \ln N+c(N)\right)+\frac{l}{8} \sum_{k=1}^{N-1} \frac{d_{k}}{N \sin \left(\frac{\pi k}{2 N}\right)},
\end{aligned}
$$

where

$$
\begin{gathered}
d_{k}=\sum_{n=1}^{N-1} \frac{2}{N} \sin ^{2}\left(\frac{\pi k n}{N}\right) \widehat{U}(n) \stackrel{a \rightarrow 0}{\longrightarrow} \frac{2}{l} \int_{0}^{l} \sin ^{2}\left(\frac{\pi k x}{l}\right) \widehat{U}(x) d x \\
\lim _{k \rightarrow \infty} d_{k}=0
\end{gathered}
$$


The second term in the second equality of eq. (C.19) is finite for $a \rightarrow 0$, while the first term is logarithmically divergent.

Now we have what we need write down the divergent terms in $E(\lambda)$

$$
E(\lambda)=\frac{2 l}{\pi a^{2}}-\frac{1}{2 a}-\frac{\lambda}{4 \pi} \ln a \int_{0}^{l} d x U(x)+\text { terms finite for } a \rightarrow 0 .
$$

Terms of $\mathcal{O}\left(\lambda^{2}\right)$ and higher do not contain $a \rightarrow 0$ divergences. This can be checked numerically. For $U(x)=m^{2}$ we recover eq. (3.17). Note that on the lattice one can replace

$$
\int_{0}^{l} U(x) d x \longrightarrow \frac{1}{2}\left(\sum_{n=0}^{N-1}+\sum_{n=1}^{N}\right) U(n) a .
$$

The difference is at most of $O(a)$ and, because $a \ln a \rightarrow 0$ in the continuum limit, this difference is irrelevant.

$\left\langle T^{11}\right\rangle$ :

For the lattice $\left\langle T^{11}\right\rangle$ we have from eq. (2.77)

$$
\begin{aligned}
\left\langle T_{n^{\prime}}^{11}\right\rangle(\lambda) & =\sum_{k=1}^{N-1} \frac{\left(\varepsilon_{k}^{2}(\lambda)-\lambda U\left(n^{\prime}\right)\right) v_{n^{\prime}}^{k}(\lambda)^{2}}{2 a \varepsilon_{k}(\lambda)} \\
& +\sum_{k=1}^{N-1} \frac{v_{n^{\prime}+1}^{k}(\lambda)^{2}-2 v_{n^{\prime}}^{k}(\lambda)^{2}+v_{n^{\prime}-1}^{k}(\lambda)^{2}}{8 a^{3} \varepsilon_{k}(\lambda)} \\
& =\left\langle T_{n^{\prime}}^{11}\right\rangle_{n^{\prime}}^{(0)}+\lambda\left\langle T_{n^{\prime}}^{11}\right\rangle_{n^{\prime}}^{(1)}+\lambda^{2}\left\langle T_{n^{\prime}}^{11}\right\rangle_{n^{\prime}}^{(2)}+\cdots .
\end{aligned}
$$

From eq. (3.33) the unperturbed tensor is

$$
\left\langle T_{n^{\prime}}^{11}\right\rangle^{(0)}=\frac{2 \pi}{a^{2}}-\frac{\pi}{24 l^{2}}+f\left(l, n^{\prime}, a\right)+\mathcal{O}\left(a^{2}\right)
$$

where $f$ is the function $(B .19)$. The first order correction to this is found by substituting eqs. (C.5), (C.6) and (C.11) into (C.23)

$$
\begin{aligned}
\left\langle T_{n^{\prime}}^{11}\right\rangle^{(1)}=\sum_{k=1}^{N-1} & {\left[\frac{\left(w_{n^{\prime}}^{k}\right)^{2}}{2 a}\left(\frac{\varepsilon_{k}^{2(1)}-U\left(n^{\prime}\right)}{\omega_{k}}-\varepsilon_{k}^{(1)}\right)+\frac{\omega_{k}}{a} w_{n^{\prime}}^{k} v_{n^{\prime}}^{k(1)}\right] } \\
& +\sum_{k=1}^{N-1}\left(-\frac{\left(w_{n^{\prime}+1}^{k}\right)^{2}-2\left(w_{n^{\prime}}^{k}\right)^{2}+\left(w_{n^{\prime}-1}^{k}\right)^{2}}{8 a^{3} \omega_{k}^{2}} \varepsilon_{k}^{(1)}\right. \\
& \left.+\frac{w_{n^{\prime}+1}^{k} v_{n^{\prime}+1}^{k(1)}-2 w_{n^{\prime}}^{k} v_{n^{\prime}}^{k(1)}+w_{n^{\prime}-1}^{k} v_{n^{\prime}-1}^{k(1)}}{4 a^{3} \omega_{k}}\right) .
\end{aligned}
$$

Next we introduce the potential

$$
\widetilde{U}(n) \equiv U(n)-U\left(n^{\prime}\right), \quad \text { i.e. } \quad \widetilde{U}\left(n^{\prime}\right)=0
$$


which leads to

$$
\begin{aligned}
\varepsilon_{k}^{2(1)} & =\tilde{\varepsilon}_{k}^{2(1)}+U\left(n^{\prime}\right), \\
\varepsilon_{k}^{(1)} & =\tilde{\varepsilon}_{k}^{(1)}+\frac{U\left(n^{\prime}\right)}{2 \omega_{k}}, \\
v_{n^{\prime}}^{k(1)} & =\tilde{v}_{n^{\prime}}^{k(1)} .
\end{aligned}
$$

Here the quantities with tildes are defined with $\widetilde{V}$ in place of $V$. Continuing with this tilde notation we find for the first-order correction to $\left\langle T^{11}\right\rangle^{(0)}$

$$
\begin{array}{rlr}
\left\langle T_{n^{\prime}}^{11}\right\rangle^{(1)} & =\left\langle\widetilde{T_{n^{\prime}}^{11}}\right\rangle^{(1)}-\frac{U\left(n^{\prime}\right)}{8} \sum_{k=1}^{N-1} \frac{1}{N \sin \left(\frac{\pi k}{2 N}\right)}+\frac{U\left(n^{\prime}\right)}{8} \sum_{k=1}^{N-1} \frac{1}{N} \sin \left(\frac{\pi k}{2 N}\right) \cos \left(\frac{2 \pi k n^{\prime}}{N}\right) \\
& =\left\langle\widetilde{T_{n^{\prime}}^{11}}\right\rangle^{(1)}-\frac{U\left(n^{\prime}\right)}{8} \sum_{k=1}^{N-1} \frac{1}{N \sin \left(\frac{\pi k}{2 N}\right)}+\frac{U\left(n^{\prime}\right)}{8} h\left(N, n^{\prime}\right), & \text { (C. } 28)
\end{array}
$$

where $h\left(N, n^{\prime}\right)$ is the function eq. (B.10).

The lattice quantity $\left\langle\widetilde{T}_{n}^{\prime \prime 1}\right\rangle^{(1)}$ remains finite in the limit $a \rightarrow 0$ and is the first-order correction to $\left\langle T^{11}\right\rangle^{(0)}$ for a lattice potential $\widetilde{V}(n)$ which vanishes at the point $n=n^{\prime}$. It is therefore relatively obvious that $\left\langle\widetilde{T}_{n^{\prime}}^{11}\right\rangle^{(1)}$ contains no $a \rightarrow 0$ divergent terms because the lattice should be (in first order) proportional to $\widetilde{V}\left(n^{\prime}\right)$. For $V(x)=m^{2}$ eq. (C.28) reduces to the system discussed in sec. 4.2. From (C.24), (C.28) we can read off the divergent terms in eq. (C.23)

$$
\left\langle T_{n}^{11}\right\rangle(\lambda)=\frac{2 \pi}{a^{2}}+f(l, n, a)+\frac{U(n)}{4 \pi} \ln a+\cdots .
$$

Second-order corrections need not be discussed. Numerical probes show that all divergent terms are displayed in eq. (C.29). 


\section{References}

[1] H. J. Rothe, Lattice gauge theories: an introduction, World Scientific, Singapore, 1992.

[2] A. Actor and I. Bender, Hard, semihard and soft boundary conditions in quantum field theory under the influence of external conditions, ed. M. Bordag, Teubner, Stuttgart, 1996.

[3] C. Alberich, Die Rolle von Randbedingungen in der skalaren Feldtheorie auf dem Gitter, Diplomarbeit, Universität Heidelberg, 1994.

[4] A. Actor and I. Bender, Phys. Rev. D52 (1995), 3581.

[5] A. Actor and I. Bender, Casimir effect with a semihard boundary, and vacuum distortion by a semihard boundary, articles in progress.

[6] M. Bordag and J. Lindig, J. Phys. A: Math. Gen. 29 (1996) 4481.

[7] I.S. Gradshteyn and I.M. Ryzhik, Table of integrals, series and products, Academic, New York, 1980.

[8] V.M. Mostepanenko and N.N. Trunov, The Casimir effect and its applications, Oxford University Press, Oxford, 1997.

[9] J. Ambjørn and S. Wolfram, Ann. Phys. (N.J.) 147 (1983) 1.

[10] A. Actor and I. Bender, Fortschr. der Physik 44 (1996) 281.

[11] H.M. Edwards, Riemann's Zeta function, Academic Press, New York, 1974.

[12] D. Deutsch and P. Candelas, Phys. Rev. D20 (1979) 3063.

[13] A. Messiah, Quantum mechanics, vol. 2, Wiley, New York,1962.

[14] S.G. Mamaev and N.N. Trunov,Yadernaya Fizika 35 (1982) 1049 [Sov. J. Nucl. Phys. 35 (1982) 612].

[15] S.G. Mamaev and N.N. Trunov, Izvestiya Vuzov. Seriya Fizika (5) (1983) 29 [Sov. Phys. J. 26 (1983) 445].

[16] G. Plunien, B. Müller and W. Greiner, Physics Reports C134 (1986) 87.

[17] V. Privman, editor, Finite size scaling and numerical simulation of statistical systems, World Scientific, Singapore, 1990.

[18] J.L. Cardy, editor, Finite size scaling, North Holland, Amsterdam, 1988.

[19] M. Krech, The Casimir effect in critical systems, World Scientific, Singapore, 1994. 